\title{
The Anticonvulsant Effects of Docosahexaenoic Acid in Rodents
}

By

Marc-Olivier Trépanier

A thesis submitted in conformity with the requirements for the degree of Masters of Science Graduate Department of Pharmacology and Toxicology University of Toronto

(C) copyright by Marc-Olivier Trépanier 2011 


\title{
University of Toronto
}

Title: The Anticonvulsant Effects of Docosahexaenoic Acid in Rodents

Degree and Convocation: M.Sc., Nov. 2011

Name: Marc-Olivier Trépanier

Department: Pharmacology and Toxicology

\begin{abstract}
Introduction: One potential new therapy for epilepsy involves the omega-3 polyunsaturated fatty acids (PUFAs), and more specifically docosahexaenoic acid (DHA).

Methods: The anticonvulsant properties of the n-3 PUFAs were assessed in a series of different experiments. Subjects received chronic dietary supplementation, sub-chronic and acute injections of either fish oil (chronic) or DHA (sub-chronic, acute). Animals were tested in the electrical afterdischarge thresholds (ADTs) model in the amygdale and the maximal pentylenetetrazol (PTZ) model.
\end{abstract}

Results: Chronic, sub-chronic, and acute administrations of n-3 PUFAs were anticonvulsant in both the electrical stimulation and maximal PTZ models. In chronic experiments, amygala ADTs increased following 3 months of fish oil administration. Fourteen days of DHA i.p. injections increased latencies to maximal PTZ seizures. Acute injection of DHA s.c. and i.v. increased unesterified serum DHA and seizure latency.

Conclusions: The present research suggests that n-3 PUFAs, and more specifically DHA, have anticonvulsant effects in vivo. 


\section{ACKNOWLEDGEMENTS}

First and foremost, I would like to thank my supervisor Dr. W.M. Burnham for this wonderful opportunity to perform research in his laboratory. Thank you for all the time and guidance that you have given me throughout this project. Learning from you for the past years has been extremely rewarding.

I would also like to thank Dr. Ameer Taha. Thank you for your guidance early on during the project and our continuous collaboration over the years. Your insights on this project have been extremely helpful and working with you was a pleasure.

Special thanks to my M.Sc. advisor Dr. Richard Bazinet. Thank you for your guidance throughout this project and our interesting discussions, through which I have learned a lot over the years.

Many thanks must also go to my laboratory partners, especially Chuck Chen, Anthony Domenichiello, Sarah Orr, Brian Scott and Jerome Cheng. Thank you for all the help around the laboratory and for the long hours put in on my experiments. Most of all, thank you for making the working environment a pleasant one to work in.

I would like to thank the following project students - Flaviu Coibanu (chapter 3,5), Henry Zeng (chapter 5), George Tchkhartichvili (Chapter 5), Joonbum Lim (Chapter 4), Terence Kai (Chapter 4), Tara Mansoursadeghi, Bryan Fong, Keiman Kwong (Chapter 6,7), and Rebecca Mantha (Chapter 7) - that have helped me throughout this research. You have been instrumental in the completion of this project

The Bahen Chair is to be thanked for the funding of this project. 
Finally, I want to thank my family and friends for all the love and support you have given me throughout the years. To my aunt Louise Côte and uncle Pierre Barette, thank you for the support over the years and being my home away from home when my parents moved back to Quebec. To my sister Sarah, thank you for the support and the interesting scientific conversation I can have with you in a family setting. To my parents Francine and Mario, thank you for everything. Words cannot describe the gratitude I have for you. I would not have been able to complete this without your support. I dedicate this thesis to you. 


\section{TABLE OF CONTENTS}

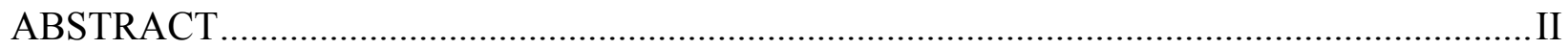

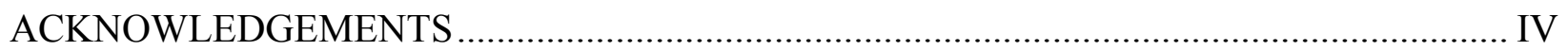

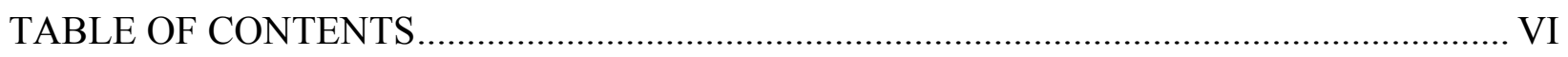

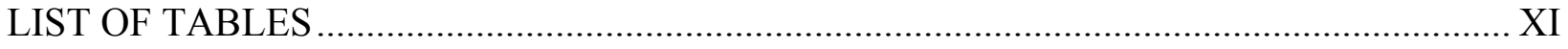

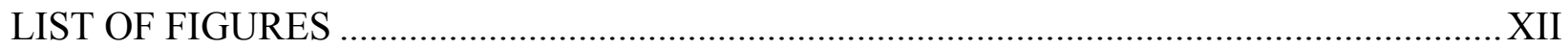

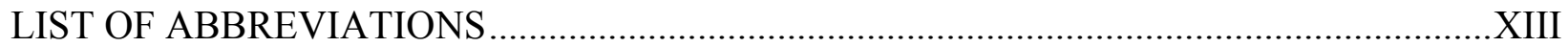

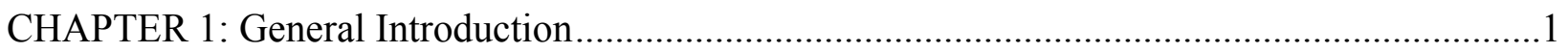

1.1 Epilepsy

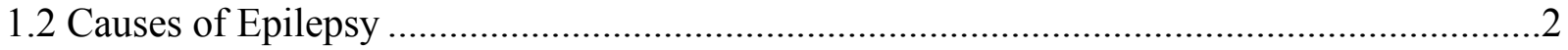

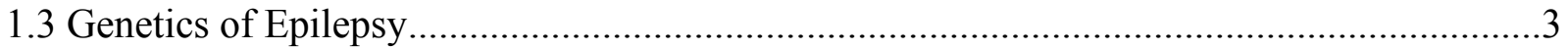

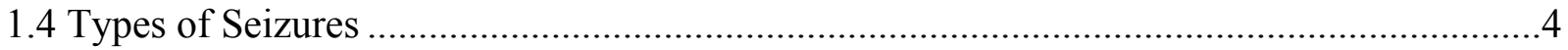

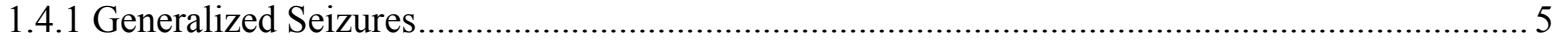

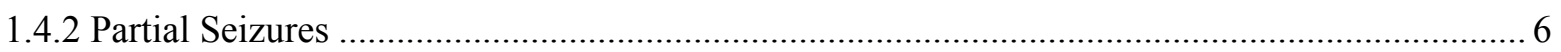

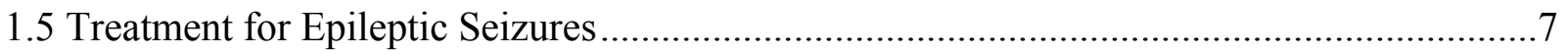

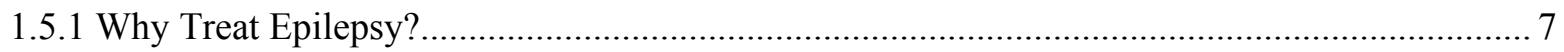

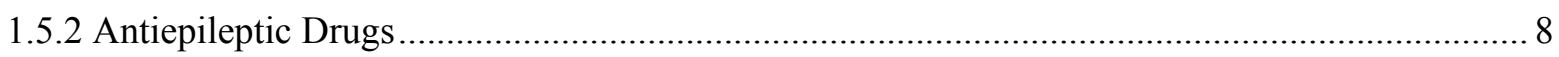

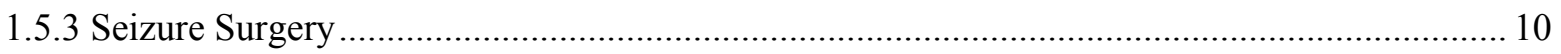

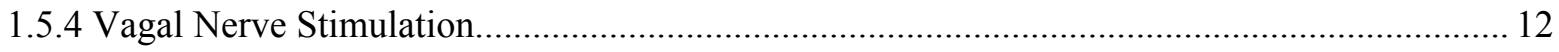

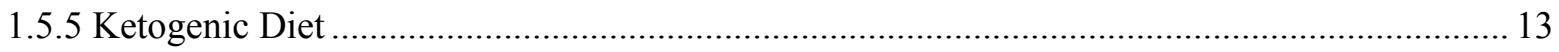

1.6 Omega-3 Supplementation - a Novel Therapy for Epilepsy ………………………...........15

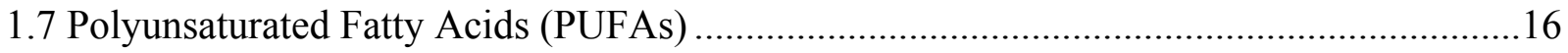

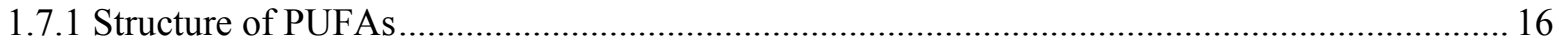

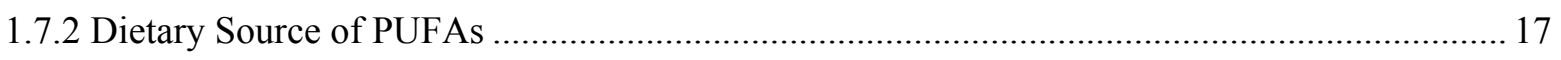

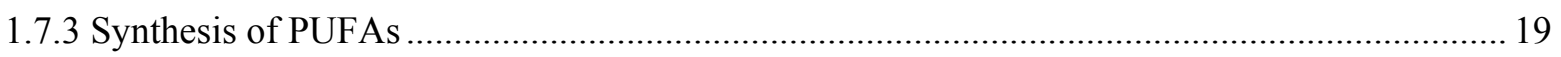

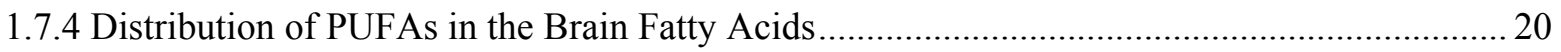

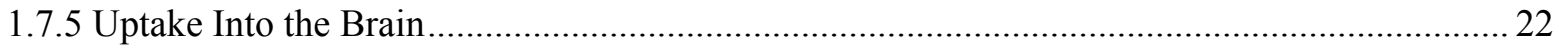

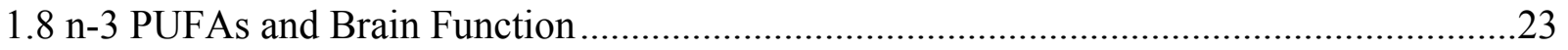

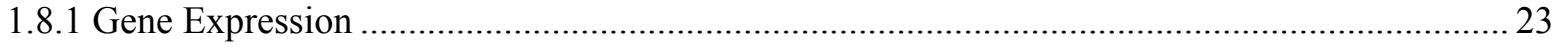

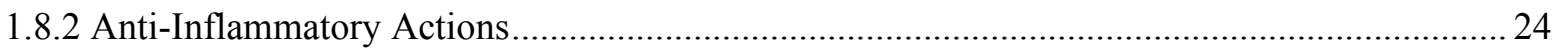

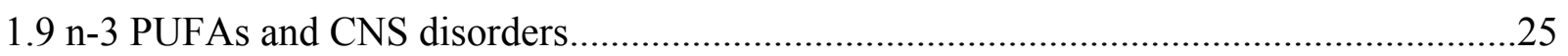

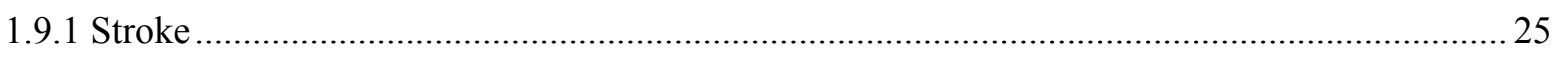

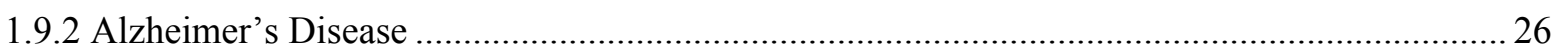




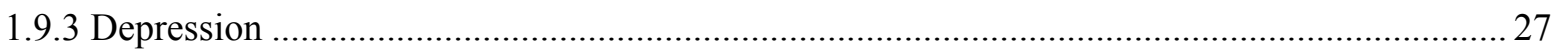

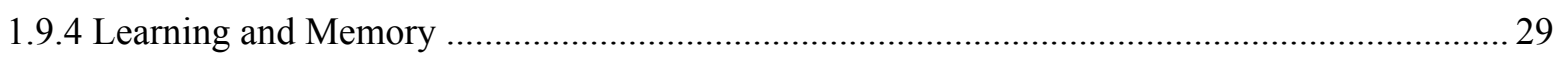

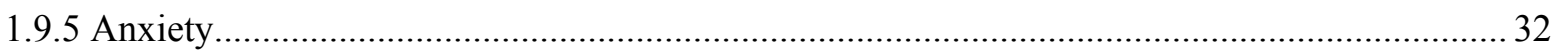

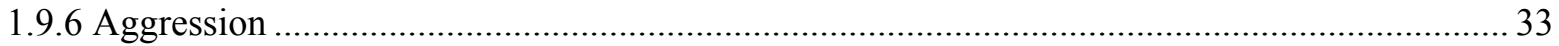

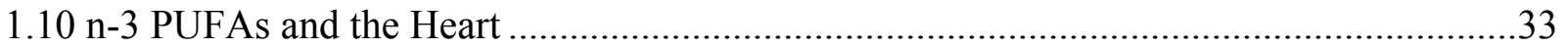

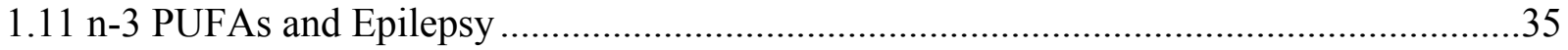

1.11.1 In Vitro Evidence for the Anticonvulsant Effects of the n-3 PUFAs ................................... 35

1.11.2 Evidence for the Anticonvulsant Effects of the n-3 PUFAs in Animal Studies ..................... 36

1.11.3 Clinical Evidence for the Anticonvulsant Effects of the n-3 PUFAs..................................... 42

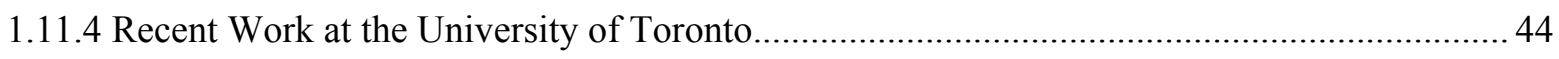

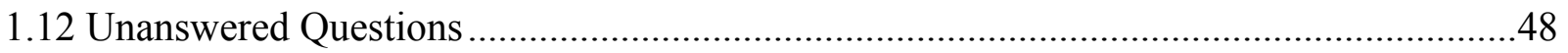

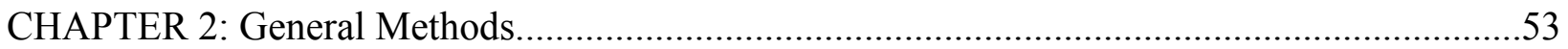

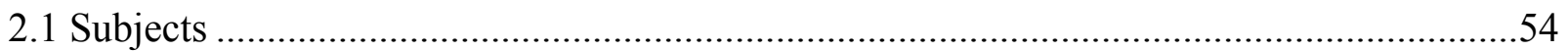

2.2 Drugs and Preparation of Drug Solutions ...................................................................54

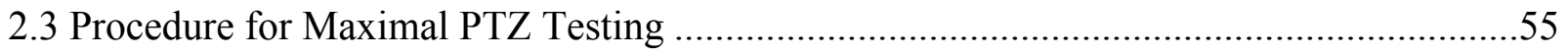

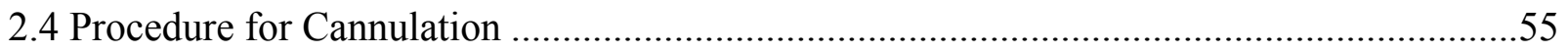

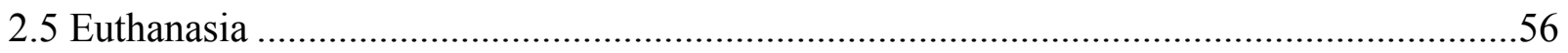

2.6 Serum Phospholipid, Triglyceride, Cholesteryl Ester and Unesterified Free Fatty Acid

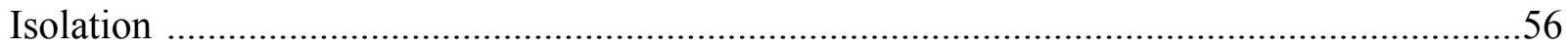

2.7 Fatty Acid Methyl Ester (FAME) Analysis by Gas-Chromatography ............................57

CHAPTER 3: Anticonvulsant Effects of Chronic Fish Oil Administration in the Electrical Afterdischarge Threshold Model in the Amygdala ................................................................5

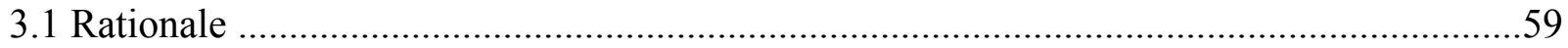

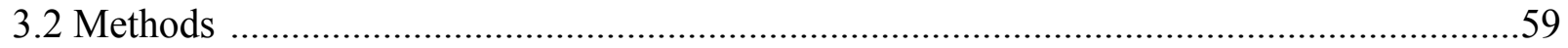

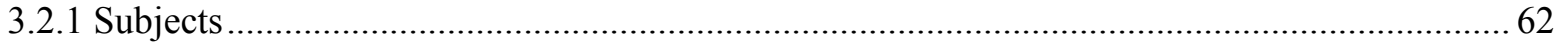

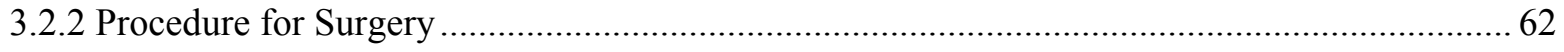

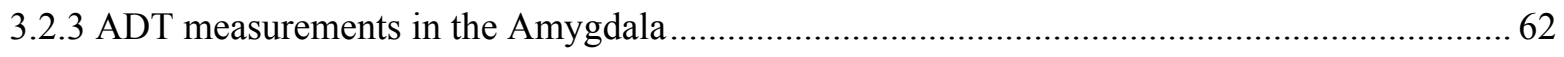

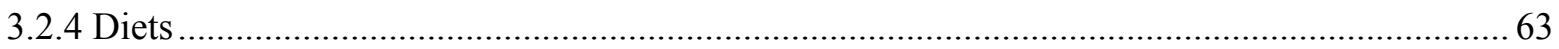

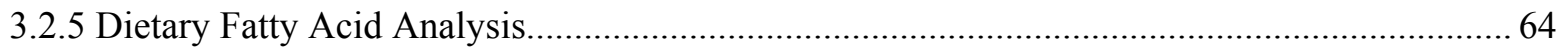

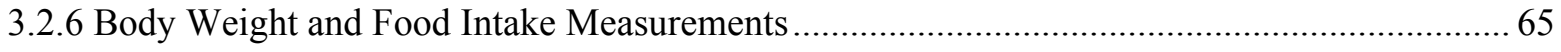

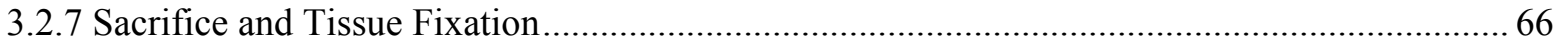

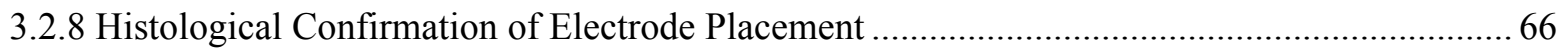




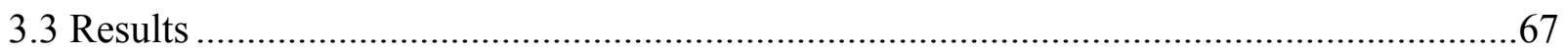

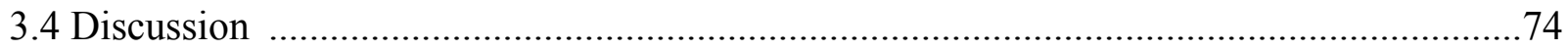

CHAPTER 4: Anticonvulsant Effects of Sub-Chronic Administration of DHA and DHA EE in the Maximal PTZ Seizure Model ..................................................................................... 77

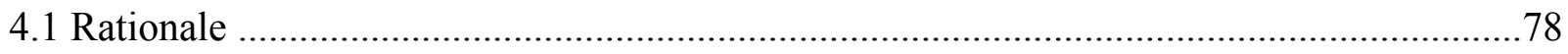

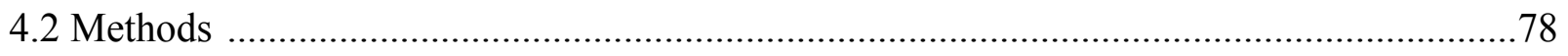

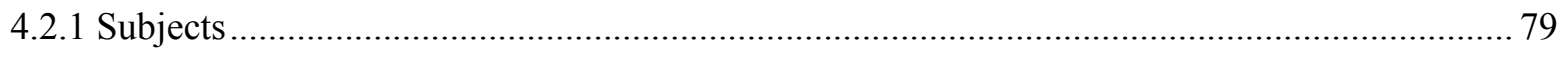

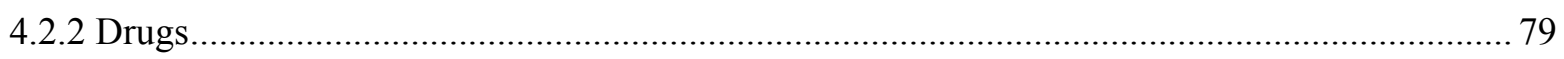

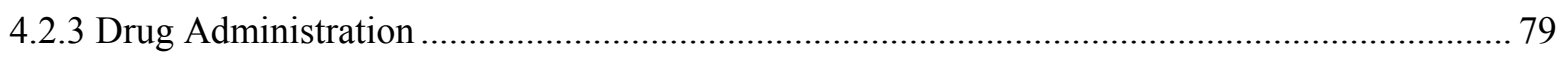

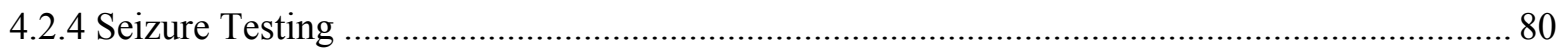

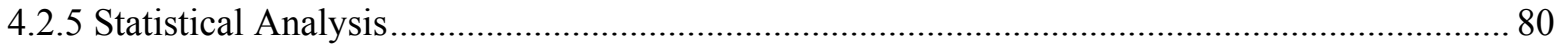

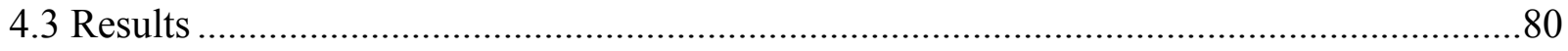

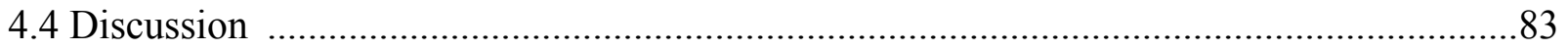

CHAPTER 5: Replication of the Inverted U Dose Response Curve for Acutely Administered DHA in the Maximal PTZ Seizure Model with Measures of Blood Levels of DHA .................85

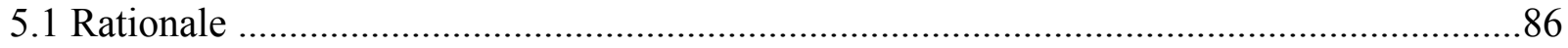

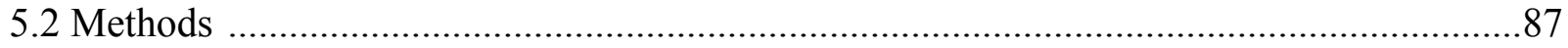

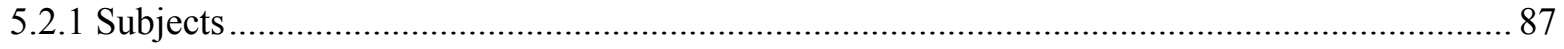

5.2.2 Drugs and Seizure Testing - Experiment 5A.................................................................... 87

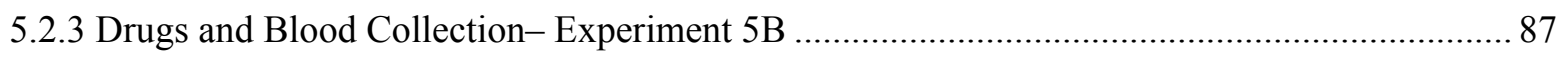

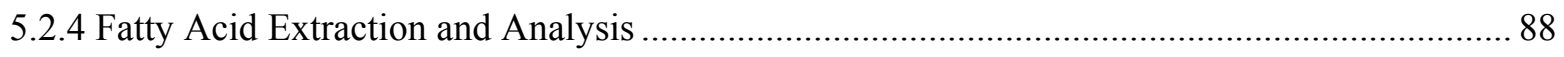

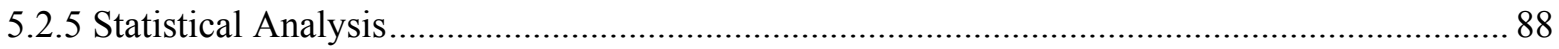

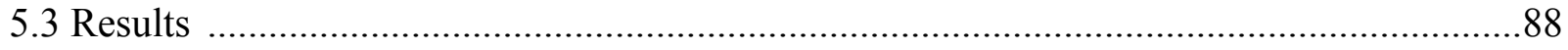

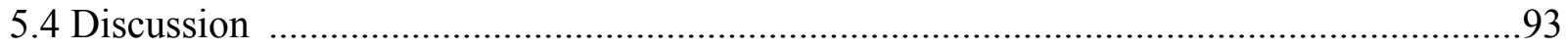

CHAPTER 6: Dose- and Time-Concentration Curves Following i.v. DHA Infusion ..................95

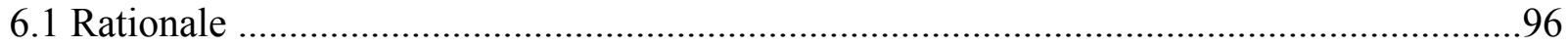

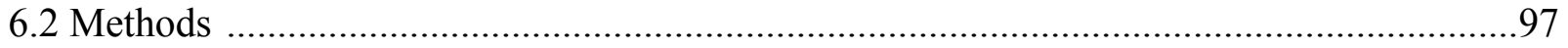

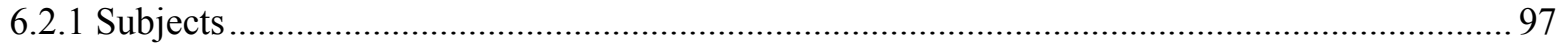

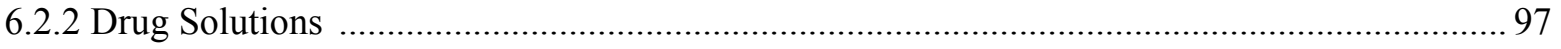




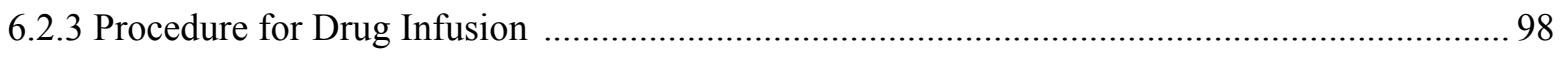

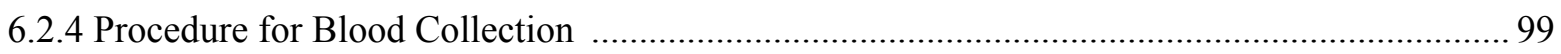

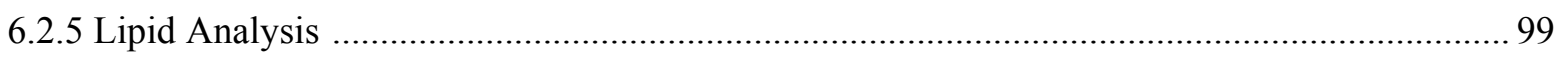

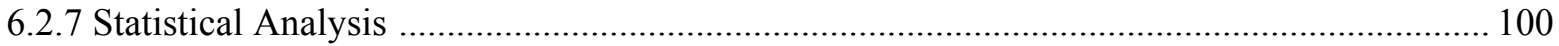

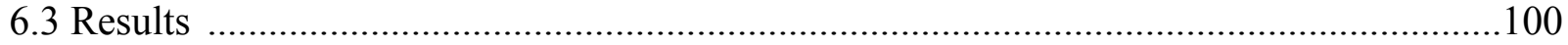

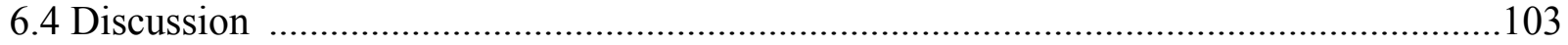

CHAPTER 7: Dose- and Time-Response Relationship of the Anticonvulsant Effects of Infused DHA in the Maximal PTZ Seizure Model ...............................................................................106

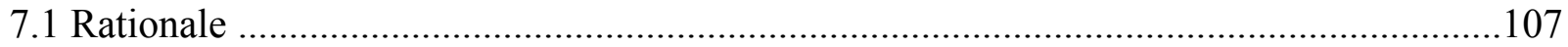

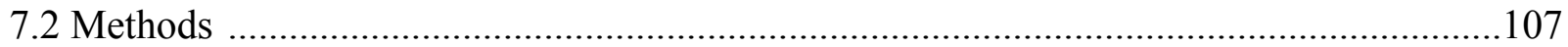

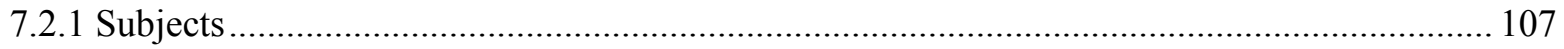

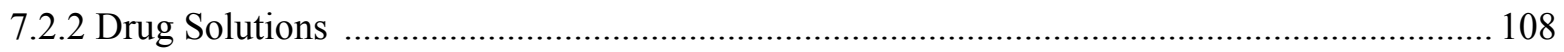

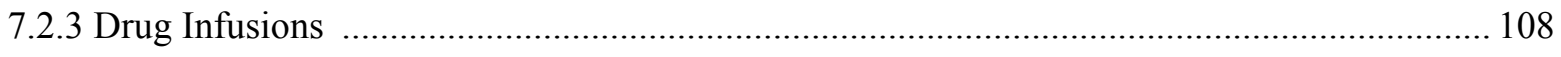

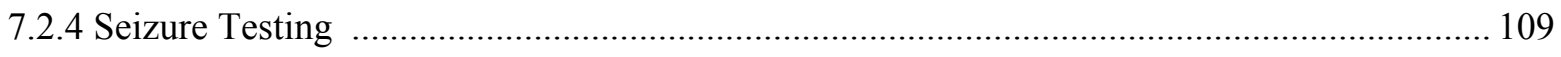

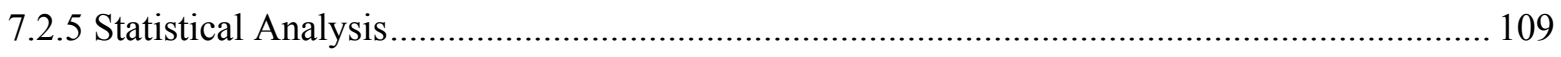

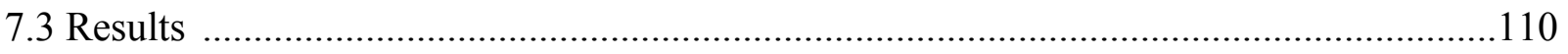

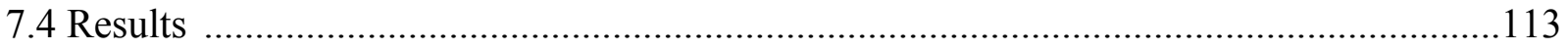

CHAPTER 8: General Discussion ..............................................................................................120

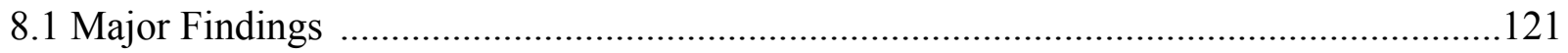

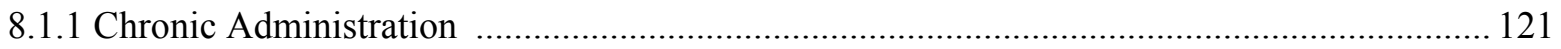

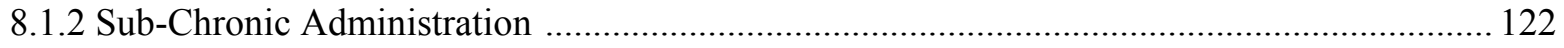

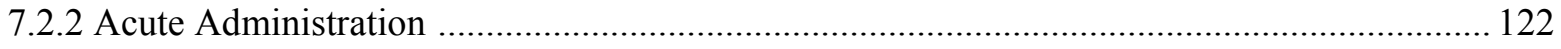

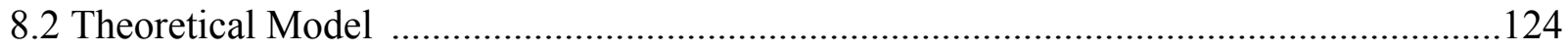

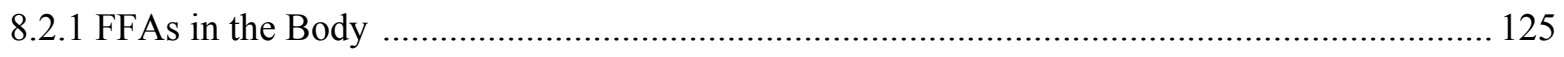

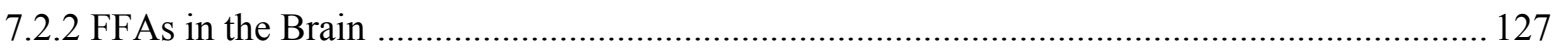

8.2.3 Chronic Oral Administration vs. Acute Systemic Administration ........................................ 128

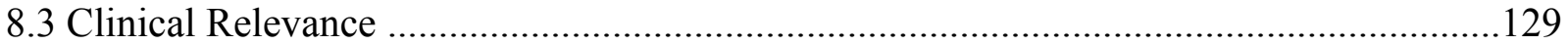

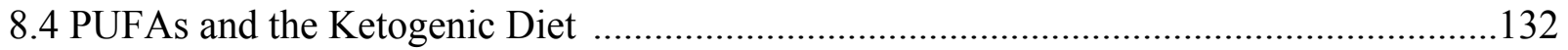

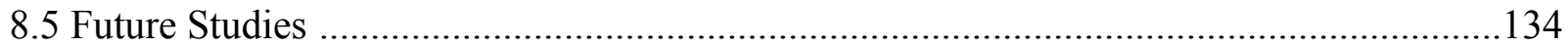

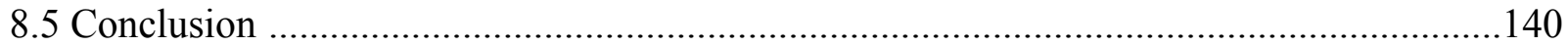




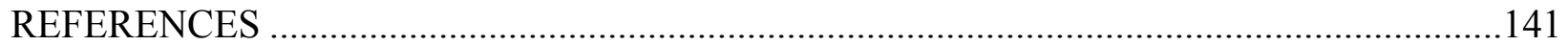




\section{LIST OF FIGURES}

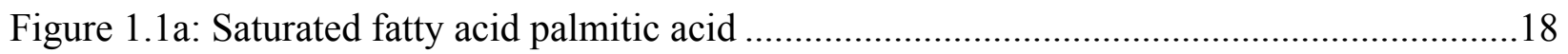

Figure 1.1b: Monosaturated fatty acid oleic acid .................................................................18

Figure 1.1c: omega-3 polyunsaturated fatty acid - docosahexaenoic acid ..................................18

Figure 1.1d: omega-6 polyunsaturated fatty acid - arachidonic acid ..........................................18

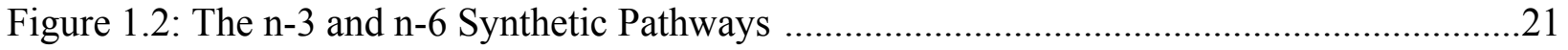

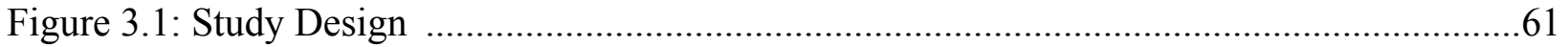

Figure 3.2: Effect of Chronic Fish Oil Administration on Weight ............................................68

Figure 3.3: Effect of Chronic Fish Oil Administration on Daily Food Consumption ...................69

Figure 3.4: Effect of Chronic Fish Oil Administration on Amygdala ADT ..................................71

Figure 3.5: Effect of Chronic Fish Oil Administration on Duration of Afterdischarges ................72

Figure 3.6: Effect of Chronic Fish Oil Administration on Behavioural Seizure Severity ..............73

Figure 4.1: Effect of Chronic Administration of DHA and DHA EE on Weight of Male Wistar

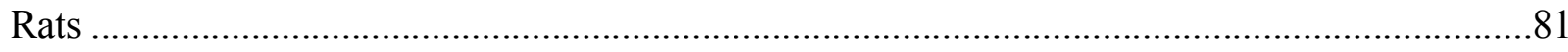

Figure 4.2: Anticonvulsant Effects of 14 Days of Chronic Administration of DHA and DHA EE

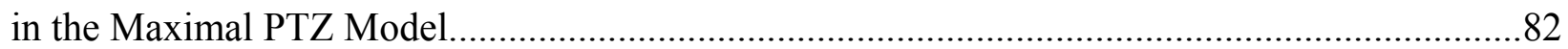

Figure 5.1: s.c. DHA Dose-Response Curve in the Maximal PTZ Model ....................................90

Figure 5.2: Unesterified DHA Concentrations in Serum Following s.c. OA or DHA Injections .91

Figure 5.3: Esterified DHA Concentrations in Serum Following s.c. OA or DHA Injections......92

Figure 6.1: Dose-Concentration Curve of Serum Unesterified DHA Concentrations..................101

Figure 6.2:Rapid Disappearance of Unesterified DHA from Serum Following Discontinuation of

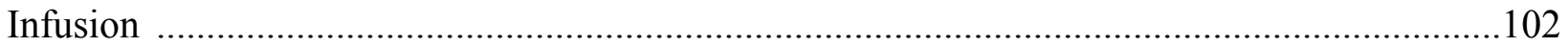

Figure 7.1:Slow DHA Infusion Dose-Response Curve in the Maximal PTZ Model .................111

Figure 7.2:Slow DHA Infusion Dose-Response Curve in the Maximal PTZ Model

(Scatter Plot)

Figure 7.3:Time-Response Curve for DHA Infusion in the Maximal PTZ Model

Figure 7.4:Time-Response Curve for DHA Infusion in the Maximal PTZ Model

(Scatter Plot)

Figure 8.1: Fate of PUFAs Following Oral Intake 


\section{LIST OF TABLES}

Table 1.1: Evidence for the Anticonvulsant Effects of the n-3 PUFAs in Animal Studies ...........39

Table 1.2: Clinical Studies Evaluating Anticonvulsant Effects of the n-3 PUFAs .....................43

Table 3.1: Composition of the Control and Experimental Diets .............................................64

Table 3.2: Percent Fatty Acid Composition of Diets ............................................................65

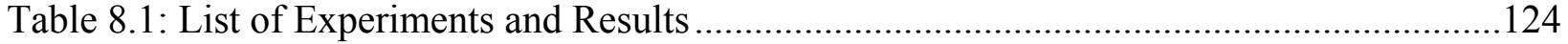




\section{LIST OF ABBREVIATION}

AA: Arachidonic acid

AD: Alzheimer's Disease

AED: Antiepileptic drugs

ALA: Alpha-linolenic acid

ANOVA: Analysis of variance

AP: Action potential

A $\beta$ : Amyloid Beta

BDNF: Brain derived neurotrophic factor

BSA: Bovine serum albumin

COX: Cyclooxygenase

CBZ: Carbamazepine

CFS: Cerebrospinal fluid

CM: Chylomicron

CNS: Central nervous system

DHA: Docosahexaenoic acid

EE: Ethyl ester

EEG: Electroencephelogram

EPA: Eicosapentaenoic acid

FAMEs: Fatty acid methyl esters

FFA: Free fatty acids

FST: Porsolt forced swim test

GABA: Gamma-Aminobutyric acid

GC: Gas-chromatography 
i.c.v.: Intracerebroventricular

ILAE: International League Against Epilepsy

i.p.: Intraperitoneal

iPLA: Calcium independent phospholipase

i.v.: Intravenous

LA: Linoleic acid

LC: Locus coeruleus

LPL: Lipoprotein lipase

MCT: Medium Chain triglyceride

MES: Maximal electroconvulsive shock

mg: Milligrams

ml: Millilitres

MWM: Morris water maze

NPD1: Neuroprotectin D1

n-3 PUFAs: Omega-3 polyunsaturated fatty acids

OA: Oleic acid

PA: Palmitic acid

PTZ: Pentylenetetrazol

PUFA: Polyunsaturated fatty acids

RvD: D-Resolvin

RvE: E-Resolvin

s.c.: Subcutaneous

VDSC: Voltage-dependent sodium channel 
VNS: Vagus nerve stimulation 


\section{CHAPTER 1}

\section{General Introduction}




\subsection{Epilepsy}

Epilepsy is one of the most common of the neurological disorders, affecting approximately 1\% of the world's population (Guberman and Bruni, 1999; Burnham, 2007; Engel and Pedley, 2008). It involves spontaneous and recurrent seizures.

A seizure is a period of synchronous, hyperexcitable neuronal firing, during which a patient often suffers a loss of consciousness or a change in mental state, and during which a patient may exhibit convulsions (Burnham, 2007).

To be classified as epilepsy, seizures must be both spontaneous (e.g., not caused by a fever, etc.) and repeated. About $10 \%$ of the world's population will have at least one seizure by the age of 80 , but, until a second seizure occurs, the patient is not diagnosed as epileptic (Guberman and Bruni, 1999).

\subsection{Causes of Epilepsy}

The epilepsies are classified as either "symptomatic" or "idiopathic". Symptomatic epilepsies have a clear-cut and demonstrable cause. The common causes for symptomatic seizures include head trauma, strokes, tumours, central nervous system infections and degenerative brain diseases - with cerebrovascular disease being the most frequent (Banerjee and Hauser, 2008). Symptomatic epilepsies make up approximately 30\% of all the epilepsies that occur (Burnham, 2007).

The remaining $70 \%$ of epilepsy cases are idiopathic, meaning that there is no demonstrable, clear-cut cause. In the idiopathic epilepsies, the brain appears normal and 
without any demonstrable abnormality. It is suspected that the idiopathic epilepsies may have a genetic cause (Burnham, 2007).

\subsection{Genetics of Epilepsy}

Even though idiopathic epilepsy is suspected to have a genetic cause, the relationship between genotype and phenotype is not entirely clear.

There are some "single-gene" epilepsies, where single gene mutations can cause epilepsy syndromes. Mutations involving ion channels ("channelopathies"), for instance causing either an increase in excitatory conductance or a decrease in inhibitory conductance can result in brain hyperexcitability (Macdonald et al., 2010).

Mutations in both the voltage-dependent sodium (VDSC) and potassium channels (VDKC) have been associated with idiopathic epilepsies (Meisler and Kearney, 2005; Hahn and Neubauer, 2009; Catterall et al., 2010). In particular, a missense mutation of the heteromer KCNQ3 of the potassium channel has been linked with idiopathic generalized epilepsy (Neubauer et al., 2008). Also, approximately $70 \%$ of patients with Dravet syndrome have a mutation in the $\alpha 1$ subunit of the VDSC (SCN1A) (Catterall et al., 2010). In $90 \%$ of these Dravet cases, the mutations arise de novo (Meisler and Kearney, 2005).

In addition, mutations involving the gamma aminobutyric acid (GABA)-A receptor, the major inhibitory receptor in the brain, have been linked with epilepsy syndromes, including childhood absence seizure, febrile seizures, generalized seizures and sporadic myoclonic epilepsy of infancy (Dravet syndrome) (Harkin et al., 2002; Marini et al., 2003). Febrile 
seizures also appear to be related to a mutation in the $\gamma 2$ subunit of the GABA-A receptor, although this mutation needs to be combined with another mutation in order to produce the childhood absence seizure phenotype (Marini et al., 2003).

Despite the fact that there are a number of single-gene epilepsies, for the most part, idiopathic epilepsy is associated with polygenetic influences. In these cases, three or more mutated genes are required to produce an epileptic phenotype. Moreover, these genes may have reduced penetrance, where they produce only a small effect, which together raises the risk of developing epilepsy in combination with environmental factors. Inheritance in these cases does not follow strict Mendellian rules, although such epilepsies may "run in families" (Ottman and Winawer, 2008).

\subsection{Types of Seizures}

In 1981, the International League Against Epilepsy (ILAE) developed a new system to classify seizures, called the Classification of Epileptic Seizures (ILAE, 1981). This new classification system is widely accepted, although certain modifications have been suggested (Engel, 1998). Under the 1981 classification system, there are two main types of seizures, generalized seizures and partial seizures (Engel et al., 2008). A “generalized" seizure involves epileptic activity in both hemisphere of the brain, while a "partial" seizure involves epileptic activity in only one hemisphere of the brain, or a part of one hemisphere.

Partial seizures can spread, however, and become generalized seizures (called "secondary generalization") (Engel et al., 2008). When a focal seizure spreads to become a more 
generalized type of seizure, the initial partial seizure is often called an "aura". Auras can range from vague to highly specific sensations, including motor and sensory sensations, which precede the seizure (Moshé and Pedley, 2008).

\subsubsection{Generalized Seizures}

There are a number of types of generalized seizures, all of which involve unconsciousness in the patient. The two types of generalized seizures seen most frequently are tonic-clonic and absence seizures. Tonic-clonic seizures (previously known as "grand-mal" seizures), are convulsive seizures, possibly originating from the brain stem (Engel et al., 2008). Tonic-clonic convulsions are accompanied by epileptic "spiking" in the electroencephalogram (EEG). Approximately $30 \%$ of patients with epilepsy have primary generalized tonic-clonic seizures (Gastaut et al., 1975; Joensen, 1986).

The second main type of generalized seizures is non-convulsive "absence" seizures (formerly known as "petit mal" seizures). Typical absence seizures involve a brief loss of consciousness, during which the patient shows 3 per second spike and wave in the EEG (Engel et al., 2008). Approximately 3to $10 \%$ of patients with epilepsy have absence seizures (Gastaut et al., 1975; Joensen, 1986).

Absence seizures may occur in combination with tonic-clonic attacks. If absence epilepsy is developed in late childhood (just pre-adolescent), there is a $90 \%$ chance of the absence seizures being mixed with tonic-clonic seizures, as compared to only $30 \%$ when the absence seizures develop in early childhood (Aicardi, 1988). 
Other (rarer) types of generalized seizures include atypical absence seizures, myoclonic seizures, tonic seizures and clonic seizures. These are often seen as a part of the catastrophic seizures of childhood epilepsies (Muthugovindan and Hartman, 2010).

\subsubsection{Partial Seizures}

Partial seizures can be subdivided into two main subtypes: simple partial seizures and complex partial seizures. During "simple" partial seizures, the patient is conscious and may experience any of a variety of sensations, perceptions or emotions. Jasper and Penfield qualified the sensations and perceptions as somatosensory, visual, auditory, vertigo, and olfactory (Penfield and Jasper, 1954). Emotions experienced often involve a feeling of fear. The lobe associated with these experiences shows epileptic discharge during the seizure (Penfield and Jasper, 1954). One study looking at the frequency of partial seizure found that approximately $60 \%$ of patients have simple partial seizures, often with secondary generalization. Only about 10 to $20 \%$ of patients experience only partial seizures without the progression to generalized seizures (Gastaut et al., 1975).

The second type of partial seizures is "complex" partial seizures (formerly known as "temporal lobe" or "psychomotor" seizures). The seizures consist of periods of "altered" consciousness, during which the patient does not convulse, but he/she is out of contact with his/her environment (Engel and Williamson, 2008). Complex partial seizures are usually preceded by a simple partial seizure or "aura". During the aura, the patient is fully conscious and often experiences a feeling of fear, sadness, anger, déjà vu, and/or jamais vu (Engel and Williamson, 2008). Subsequently, during the seizure itself, consciousness is "altered" and the 
patient ceases to be responsive to his/her environment. This altered state can be accompanied by "absent minded" behaviours called "automatisms". Automatisms include lip smacking, chewing and teeth grinding (Engel and Williamson, 2008).

Complex partial seizures are the most common type of partial seizures, with approximately $40 \%$ of adult patients having complex partial seizures as their predominant seizure (Gastaut et al., 1975).

Complex partial seizures are also the most drug resistant of the common seizure types. This may be due to the fact that drug development uses animal models for tonic-clonic seizures and absence seizures, but not for complex partial seizures. Due to this, drug developers may have missed drugs effective against complex partial seizures (Engel and Williamson, 2008).

\subsection{Treatment for Epileptic Seizures}

\subsubsection{Why Treat Epilepsy?}

Although seizures are not lethal - and patients can live normal lives between seizures it is important to treat epilepsy. Patients with uncontrolled epilepsy face discrimination and socioeconomic hardships. They face an increased risk of unemployment, for instance, and are often unemployed or underemployed (Stefanello et al., 2010; Jennum et al., 2011). Due in part to their seizures, they have a lower quality of life than most other disease groups (Balabanov et al., 2008; Jennum et al., 2011).

Patients suffering from intractable seizures are also unable to drive in most countries. This restriction may be unnecessary, since, although patients with epilepsy have increased risks 
of accidents, it is only a small increase in risk as compared to other causes (Krumholz, 2009). Despite this, epilepsy patients have their licences revoked upon their first diagnosis, and they must be seizure free for a minimum of 6 months to 1 year in order to regain their licenses (Guberman and Bruni, 1999).

Aside from the seizures, patients also often suffer from epilepsy-related psychiatric comorbidities (Guberman and Bruni, 1999). The most common psychiatric disorder affecting epileptics is depression (Guberman and Bruni, 1999; Tellez-Zenteno et al., 2007b). Suicide risk is up to 5 times higher in epileptics than in the healthy population (Guberman and Bruni, 1999; Tellez-Zenteno et al., 2007b; Stefanello et al., 2010). There are also increased risks of anxiety and psychosis associated with epilepsy (Tellez-Zenteno et al., 2007b; Stefanello et al., 2010).

Patients with seizures also often display learning disabilities which cause trouble in school. Camfield and colleagues have reported that only $60 \%$ of patients with idiopathic generalized tonic-clonic seizures graduate from high school, while $77 \%$ of them report significant learning difficulties (Camfield and Camfield, 2010).

Epilepsy, although not lethal, therefore requires therapeutic intervention. The following sections will discuss a variety of seizure therapies, including antiepileptic drugs (AED), seizure surgery, vagus nerve stimulation, and the ketogenic diet.

\subsubsection{Antiepileptic Drugs}

The treatment of choice for epilepsy is AED therapy (Burnham, 2007). There are currently 18 drugs approved for use in the United States and Canada for the treatment of 
epilepsy (Chong and Bazil, 2010). The various drugs are approved for either monotherapy or as adjunct therapy in combination with other AEDs.

For the majority of patients, the anticonvulsant medications are very effective. Approximately $60 \%$ of patients become seizure free when treated with AEDs (Burnham, 2007). Generally, the patient will first be placed on one single drug. If that drug fails to alleviate seizures, the patient will be put on a different single drug. If monotherapy fails again, the patient will then be placed on a combination of two or more AEDs in order to gain seizure control (Guberman and Bruni, 1999).

Three main mechanisms of action have been identified for AEDs (Burnham, 2007). These mechanisms are: 1) modulation of the voltage-dependent sodium channel (VDSC), 2) modulation of the t-type voltage-dependent calcium channel, and 3) modulation of the GABAergic system (Burnham, 2007; Guimaraes and Ribeiro, 2010).

The VDSC has 3 states: open, inactive and closed. During an action potential (AP), the channel goes from closed to open, allowing for the influx of sodium into the neuron and the propagation of the AP. Following this activation, the channel then becomes inactive for a few milliseconds before returning to its closed state (Guimaraes and Ribeiro, 2010). This cycle must be completed before the cell can fire again (Rogawski and Loscher, 2004). Antiepileptic drugs like phenytoin, carbamazepine and lamotrigine stabilize the channel in its inactive state for a slightly longer duration. This prolongs the refractory period and prevents the high frequency repetitive firing found during a seizure (Rogawski and Loscher, 2004; Burnham, 2007; Guimaraes and Ribeiro, 2010). Drugs which work on this channel are effective against tonic-clonic seizures and partial seizures (Rogawski and Loscher, 2004). 
Other AEDs, such as ethosuximide, interact with t-type calcium channels. T-type calcium channel currents in thalamic relay neurons are responsible for the $3 \mathrm{~Hz}$ spike and wave discharge classically seen in absence seizures. Ethosuximide reduces these t-type calcium currents (Macdonald and Rogawski, 2008).

The third main mechanism of action of AEDs is enhancement of GABA-A mediated inhibition. In the 1970s, it was discovered that drugs that reduced GABAergic inhibition resulted in lowered seizure thresholds and that drugs that increased GABAergic inhibition raised seizure thresholds (Meldrum, 2008). It was found that several AEDs already on the market at that time acted by enhancing GABA, including the benzodiazepines and barbiturates. The GABA system then became a favoured molecular target for new potential AEDs, leading to the discovery of a number of new drugs, including vigabatrin, which targets GABAtransaminase and blocks the degradation of GABA, tiagabine, which blocks GABA reuptake into the neurons, and topiramate which enhances GABA activity much as the benzodiazepines do (Faught and Glauser, 2008; Meldrum, 2008).

\subsubsection{Seizure Surgery}

As mentioned above, approximately 30 to $40 \%$ of patients are not responsive to drug therapy. For these patients, alternative medical therapies need to be considered. One of these therapies is seizure surgery. To be considered for surgery, patients need to have intractable seizures, and, most often, a well defined and identifiable focus (Guberman and Bruni, 1999). 
There are two major types of surgery, resective surgeries and functional surgeries (Duncan, 2007). "Resective" surgery involves removing an epileptic focus. Resective surgeries include anterior temporal lobe surgery, lesionectomy, neocortical resection and hemispherectomy (Duncan, 2007). Anterior temporal lobe surgery is the most common procedure, making up approximately $70 \%$ of resective surgeries (Duncan, 2007). TellezZenteno et al have found in a meta-analysis that resective surgeries resulted in $71 \%$ of patients becoming seizure free (Tellez-Zenteno et al., 2005).

"Functional" surgeries are only performed if resective surgery is not possible. They alleviate the symptoms, but do not offer much possibility of a cure. These surgeries include corpus callosotomy and subpial transection (Duncan, 2007). Generally, functional surgeries are less successful than resective surgeries. "Corpus callosotomy" involves cutting the anterior two thirds of the corpus callosum. It reduces drop attacks in only $35 \%$ of patients. "Multiple subpial transaction" involves making cuts in the cortex, and is used only on cortex that cannot be removed (e.g. motor cortex). Only $16 \%$ of patients are seizure free following subpial transection (Tellez-Zenteno et al., 2005).

Seizure surgery may improve the co-morbidities of epilepsy as well as stopping the seizures. Temporal lobe surgery is associated with a reduction in both anxiety and depression (Devinsky et al., 2005). This improvement appears to be related to the success of the surgery, with seizure free patients showing greater psychosocial improvements compared to non-seizure free patients (Tellez-Zenteno et al., 2007a). 


\subsubsection{Vagus Nerve Stimulation}

Another therapy used to treat refractory (drug resistant) epilepsy is vagus nerve stimulation (VNS). A stimulator implanted under the left clavicle is connected to a lead that stimulates the left vagus nerve (Ben-Menachem, 2002; Shafique and Dalsing, 2006). Stimulation of the vagus nerve creates changes in the brain's neuronal firing, which somehow raise seizure thresholds (Ben-Menachem, 2002). The exact mechanism of action is still unknown, but one hypothesis is that an increase in the brain's bloodflow associated with VNS may be correlated with seizure protection (Ben-Menachem, 2002; Ramani, 2008). A second hypothesis is that VNS raises seizure threshold by activating the locus coeruleus (LC). Activation of the LC has been shown to produce seizure protection (Neuman, 1986), possibly through the release of norepinephrine (Krahl et al., 1998). In a rat model, lesioning the LC with 6-hydroxydopamine attenuates the effects of VNS (Krahl et al., 1998).

VNS is generally well tolerated. There are some side effects that have been reported, however. The most commonly reported side effects have been hoarseness, coughing, voice changes and headaches (Ramsay et al., 1994; Handforth et al., 1998; Morris and Mueller, 1999). The side effects tend to disappear, however, with increasing duration of treatment (Morris and Mueller, 1999). Although the vagus nerve innervates the heart, no changes in cardiac rhythms have been seen with VNS (Ben-Menachem et al., 1994; Handforth et al., 1998).

Aside from alleviating seizures, VNS also provides improvements in both mood (Elger et al., 2000) and cognition (Clark et al., 1999). 


\subsubsection{Ketogenic Diet}

The ketogenic diet is another therapy used to treat drug resistant epilepsy. It is usually used in children and had its genesis in the observation that fasting can stop seizures.

It has been known since antiquity that fasting can alleviate the symptoms of epilepsy. A mention of this can be found in the writings of Hippocrates in the $5^{\text {th }}$ century B.C. (Stafstrom et al., 2008).

Fasting creates a state of ketosis in the bloodstream. "Ketosis" is an elevation of the ketone breakdown products of fat metabolism - acetoacetate, beta-hydroxybutyrate and acetone (Hartman et al., 2007). In 1921, Wilder, the originator of the ketogenic diet, hypothesized that a high fat diet - which also creates ketosis - could produce the same effects as fasting (Stafstrom et al., 2008). Wilder and Winter therefore developed a diet which produced ketosis by combining fat and carbohydrates in a ratio of 2:1 or greater (Wilder and Winter, 1922). This diet is now known as the "ketogenic diet", and is still used in the control of drug-resistant seizures in children.

The ketogenic diet, as used today, usually involves a ratio to $4: 1$ of fat to protein and carbohydrate combined (Freeman and Kossoff, 2010). Variations of this ratio can be made to ensure sufficient protein intake for adolescents (Kossoff et al., 2009). Other modification of the ketogenic diet include the modified Atkins Diet and the Medium Chain Triglyceride diet (MCT) (Kossoff et al., 2009).

Clinical studies have shown that the ketogenic diet has clear-cut antiepileptic properties. A recent meta-analysis found that approximately $30 \%$ of children on the ketogenic 
diet had at least a 50\% reduction in seizure frequency (Keene, 2006). An earlier meta-analysis by Lefevre and Aronson had found the ketogenic diet to be slightly more effective having a $50 \%$ reduction in seizure frequency in approximately $50 \%$ of children placed on the diet (Lefevre and Aronson, 2000).

The drawback of these earlier studies was a lack of control groups. Two studies with randomized controls have recently been published, however (Neal et al., 2008, 2009). In the first study, patients on the classic (4:1) ketogenic diet showed a 75\% decrease in seizure frequency as compared to the controls, with $35 \%$ of patients on the ketogenic diet showing at least a 50\% reduction in seizures (Neal et al., 2008). In the second study, the classic ketogenic diet was compared to the MCT ketogenic diet. No significant differences were found between the ketogenic diet and the MCT diet (Neal et al., 2009).

The ketogenic diet also has certain side effects, the most common being vomiting and gastrointestinal problems (Keene, 2006). Renal stones are also more common than in the normal populations, with one study finding $0.8 \%$ of ketogenic diet patients developing stones (Furth et al., 2000).

The mechanism of action of the ketogenic diet is still unknown. It is known, however, that the low intake of carbohydrates in the ketogenic diet shunts free fatty acids from the tricarboxylic acid cycle to the production of ketone bodies (Stafstrom et al., 2008). Several hypothetical mechanisms of action have been proposed based on this switch to a fat-based metabolism.

One of these is the "ketone hypothesis" - the hypothesis that the ketone bodies have direct anticonvulsant properties (Hartman et al., 2007). There is some support for this 
hypothesis. Acetone, for instance, has been shown to suppress seizure activity in vivo (Likhodii and Burnham, 2002; Likhodii et al., 2002; Rho et al., 2002; Likhodii et al., 2003). Acetoacetate has also been reported to have anticonvulsant properties (Rho et al., 2002). Betahydroxybutyrate, however, does not appear to be anticonvulsant (Bough et al., 1999; Rho et al., 2002). A drawback to the ketone hypothesis is that it has not yet been demonstrated that ketone bodies rise to anticonvulsant levels in the blood of children on the ketogenic diet.

Another hypothesized mechanism of action for the ketogenic diet relates to the elevation of polyunsaturated fatty acids (PUFAs). The ketogenic diet elevates blood PUFAs in both humans (Fraser et al., 2003; Dahlin et al., 2007) and in rats (Taha et al., 2005). Data indicate that PUFAs convey seizure protection, as discussed in this thesis. This will be further discussed in detail in later sections.

\subsection{Omega-3 Supplementation - a Novel Therapy for Epilepsy}

Omega-3 polyunsaturated fatty acids (n-3 PUFAs) have been suggested as an alternative treatment for epilepsy (Yehuda et al., 1994; Cunnane et al., 2002; Schlanger et al., 2002). Their possible anticonvulsant effects are the topic of this thesis.

The n-3 PUFAs are fatty acids with multiple double bonds, including one at the omega-

3 position. They are largely derived from dietary sources, such as flaxseed and fish. They are abundant in the mammalian brain and regulate many brain functions (below).

The notion that n-3 PUFAs are possibly anticonvulsant comes from the known antiarrhythmic properties that n-3 PUFAs possess. n-3 PUFAs reduces heart hyperexcitability 
(Billman et al., 1994; Kang and Leaf, 1994; Xiao et al., 1995). It has therefore been postulated that they might be anticonvulsant as both the brain and the heart are excitable tissues (Climax and Sewell, 1981; Fraser et al., 1993; Yehuda et al., 1994).

If effective, the n-3 PUFAs might offer a safe and non-toxic alternative to drugs as no side effects have been linked to the consumption of n-3 PUFAs. Furthermore, they would be cheaper than the newer anticonvulsant drugs, and they could be easily bought at drug stores without a prescription.

\subsection{Polyunsaturated Fatty Acids (PUFAs)}

\subsubsection{Structure of PUFAs}

A fatty acid is a hydrocarbon chain that has a carboxylic group at one end of the chain and a methyl terminal at the other end (Spector, 2006). A typical fatty acid is illustrated in Figures 1.1.

There are several types of fatty acids, including saturated, monounsaturated, and polyunsaturated fatty acids. "Saturated" fatty acids have no double bonds in the hydrocarbon chain (Figure 1.1a). They include palmitic acid (PA) and stearic acid. "Monounsaturated" fatty acids have a single double bond in the hydrocarbon chain. These include oleic acid (OA) (Figure 1.1b). "Polyunsaturated" fatty acids (PUFAs) have multiple double bonds in the hydrocarbon chain. The number of double bonds can vary from 2 to 6 . These include docosahexaenoic acid (DHA) and arachidonic acid (AA) (Figure 1.1c-d) (Spector, 2006). 
The PUFAs are further divided into two major groups: omega-3 and omega-6 PUFAs. The location of the first double bond in relation to the methyl terminal - referred to as the "omega carbon" - determines group membership. If the first double bond is 3 carbons removed from the omega carbon, the fatty acid is classified as an "omega-3 (n-3)" PUFA (Figure 1.1c). If the first double bond is found 6 carbons removed from the omega carbon, the fatty acid is referred as an "n-6 PUFA" (Figure 1.1d). DHA and AA are the most common n-3 and n-6 PUFAs respectively (Spector, 2006). The present thesis will focus primarily on the n-3 PUFAs.

\subsubsection{Dietary Sources of PUFAs}

The body can obtain PUFAs from multiple dietary sources. The short-chain n-3 PUFAs, including alpha linolenic acid (ALA), are found abundantly in linseed and flaxseed oils (Russo, 2009). These short-chain n-3 PUFAs can only be obtained from dietary sources, since the body cannot synthesize them de novo.

The long-chain n-3 PUFAs, such as DHA and eicosapentaenoic acid (EPA) are best obtained by eating marine plants and animals. Salmon, herring and tuna are especially rich in long-chain n-3 PUFAs (Russo, 2009). The long-chain PUFAs can be synthesized in the body to a certain extent, but synthesis is inefficient (below) and dietary sources are better (Gao et al., 2009; Gao et al., 2010).

The n-6 PUFAs are found in vegetable oils, such as corn, soybean, and sunflower oils (Russo, 2009). n-6 PUFAs are also primarily found in animal meats such as beef and chicken (Taber et al., 1998; Komprda et al., 2005; Russo, 2009). 
<smiles>CCCCCCCCCCCCCCCC(=O)O</smiles>

Figure 1.1a. Saturated fatty acid palmitic acid (image taken from lipidlibrary.aocs.org)<smiles>CCCCCCCCC=CCCCCCCCC(=O)O</smiles>

Figure 1.1b. Monosaturated fatty acid oleic acid (image taken from lipidlibrary.aocs.org)

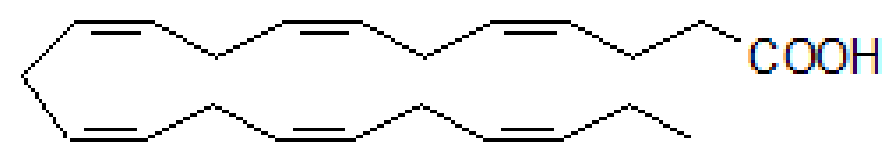

Figure 1.1c. omega-3 polyunsaturated fatty acid docosahexaenoic acid (image taken from lipidlibrary.aocs.org)

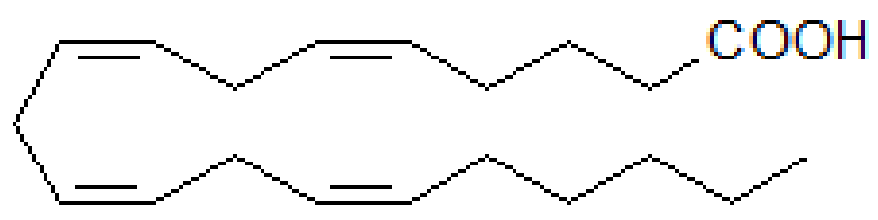

Figure 1.1d. omega-6 polyunsaturated fatty acid arachidonic acid (image taken from lipidlibrary.aocs.org) 
Humans probably evolved on a diet consisting of a ratio of n-6 to n-3 PUFAs that was approximately of 2 to 1 (Simopoulos, 2000). Today's Western diet, however, has shifted towards more n-6 PUFA consumption, shifting the ratio of n-6 to n-3 PUFAs from 2 to 1 to as high as 7-25 to 1 (Simopoulos, 2000; Meyer et al., 2003; Astorg et al., 2004; Denomme et al., 2005; Sioen et al., 2006; Flood et al., 2007; O'Sullivan et al., 2011).

Not only is the ratio of n-6 to n-3 PUFAs out of balance, the actual amount of n-3 PUFAs is low. The recommended daily intake of DHA plus EPA ranges from $0.1 \mathrm{~g}$ to $1 \mathrm{~g}$ per day (Simopoulos, 2000; Harris et al., 2009; Kris-Etherton et al., 2009; Lucas et al., 2010). Several studies, however, have shown that the Western population consumes amounts of n-3 PUFAs that are below the recommended levels (Denomme et al., 2005; Flood et al., 2007; Lucas et al., 2010; Sioen et al., 2010; O'Sullivan et al., 2011).

\subsubsection{Synthesis of PUFAs}

Although PUFAs cannot be made de novo in the body, as noted above, short chain PUFAs can be enzymatically modified into longer PUFAs (Figure 1.2). The precursor PUFA for n-6 synthesis is linoleic acid (LA), while the n-3 precursor is ALA. Both of these precursors are 18 carbon chains (Sprecher, 2000).

The elongation of these precursor molecules largely takes place within the liver. The heart and the brain do some conversion, but have much lower conversion rates since they have lower expression of the related enzymes (Rapoport et al., 2010). 
Elongation of the 18 carbon precursors (ALA and LA) is modulated by enzymes called "elongases". Elongases add carbons to 18 carbon chains, generating 20 carbon chains (such as AA and EPA) or 22 carbons chains (DHA) (Sprecher, 2000).

Following elongation by the elongases, a second type of enzyme called a "desaturase" adds double bonds to the carbon chains. The short chain precursors, ALA and LA, have 3 and 2 double bonds respectively. Desaturases add double bonds to these to give the chain 4 (AA), 5 (EPA) or 6 (DHA) double bonds (Sprecher, 2000).

As noted above, the conversion of ALA to DHA is relatively inefficient. In rats, the conversion of ALA to DHA ranges from 1.4-14\% (Cunnane and Anderson, 1997; Gao et al., 2009). In humans, the rate of conversion is also extremely low, with most studies reporting ranges from 1\% to 9\% (Burdge et al., 2002; Burdge and Wootton, 2002; Burdge et al., 2003; Plourde and Cunnane, 2007; Brenna et al., 2009). Females have a somewhat higher rate than males (Burdge and Wootton, 2002; Pawlosky et al., 2003). The conversion of n-6 PUFAs is also relatively inefficient. In rats, approximately $3 \%$ of LA is converted into AA (Cunnane and Anderson, 1997; Gao et al., 2010).

\subsubsection{Distribution of PUFAs in the Brain Fatty Acids}

The PUFAs make up approximately $30 \%$ of the total brain fatty acids (Igarashi et al., 2007). The largest contributors are DHA and AA. ALA, LA and EPA are found in small to non-existent levels in the lipid pools of the brain (Igarashi et al., 2007). The majority of DHA 
Figure 1.2: The n-3 and n-6 Synthetic Pathway
n-3 pathway
n-6 pathway

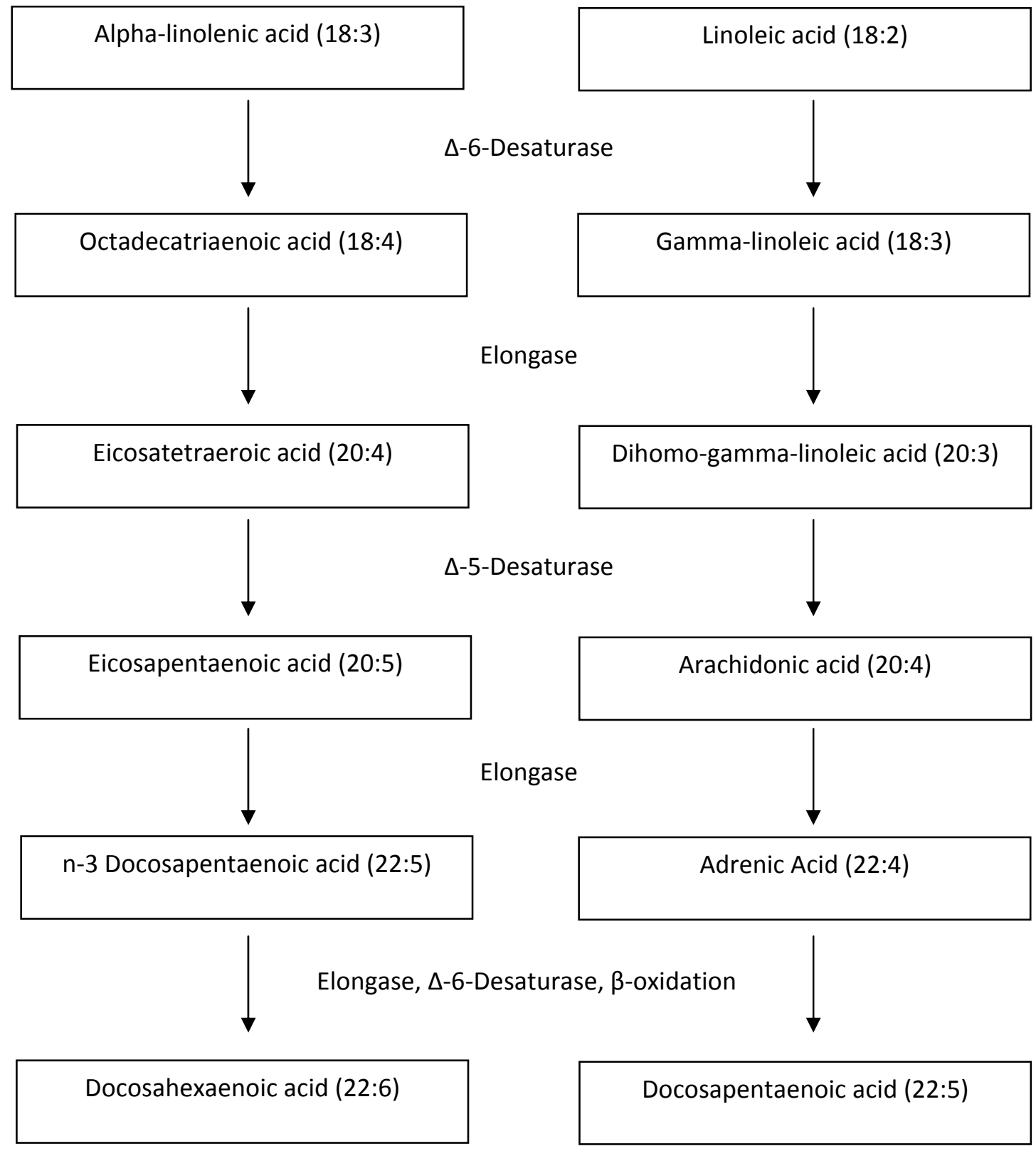


and AA are found in esterified form, forming part of the brain's phospholipid pool ( $>99 \%)$. Only very small amounts of DHA and AA are found in the triglyceride and unesterified fatty acid pools, which are also known as the "free fatty acids" (FFA) pool (Igarashi et al., 2007).

Within the brain phospholipid pool, DHA is located within certain specific phospholipids. More specifically, DHA is incorporated into phosphatidylserine and phosphatidylethanolamine (Svennerholm, 1968; Salem et al., 2001; Chen et al., 2008b). Of these, the largest phospholipid pool, however, is the phosphatidylserine pool (Svennerholm, 1968), meaning that the majority of the DHA is found in the phosphatidylserine pool (Chen et al., 2008b).

\subsubsection{Uptake Into the Brain}

Many mechanisms have been suggested to explain the uptake of lipids from the blood into the brain. Explanations have included the passive diffusion of free fatty acids (Hamilton et al., 2001) and the active transport of lipoproteins (Edmond, 2001). Recently, it has become clear that it is the free fatty acids bound to albumin in the blood that are taken up into the brain (Chen et al., 2008a), and that they are taken up by a passive diffusion mechanism and not by active transport (Ouellet et al., 2009). Although the blood-brain barrier does contain lipoprotein transport proteins, mice with these proteins knocked out do not have altered brain fatty acid levels - suggesting that active transport is not the mechanism of lipid uptake into the brain (Chen et al., 2008b; Song et al., 2010). 
Since the mechanism of lipid uptake is by passive diffusion, one must explain why DHA and AA are predominant in the brain. The answer relates to metabolism. All PUFAs are taken up equally by the brain but, with the exception of DHA and AA, are immediately beta-

oxidized once they enter the brain (Demar et al., 2005; DeMar et al., 2006a; Chen et al., 2009). EPA, for instance, is preferentially beta-oxidized, whereas DHA is preferably incorporated into the phospholipid membrane (Chen et al., 2009).

\section{8 n-3 PUFAs and Brain Function}

Once up taken into the brain, n-3 PUFAs have multiple effects on the brain function. These effects include the regulation of gene expression and the production of antiinflammatory metabolites, among many others.

\subsubsection{Gene Expression}

Omega-3 Polyunsaturated fatty acids have been shown in feeding studies to regulate the expression of many different genes (Kitajka et al., 2002; Barcelo-Coblijn et al., 2003). Kitajka et al., for instance, have shown that the chronic feeding of EPA and DHA results in the increased expression of 55 different genes, while reducing the expression of 47 others. The genes affected varied in function, including genes with functions related to the cytoskeleton, lipid metabolism, signal transduction, synaptic proteins and receptors (Kitajka et al., 2002). Brain derived neurotrophic factor (BDNF), which increases plasticity and cell survival, appears to have increased expression during n-3 PUFA supplementation (Bousquet et al., 2009), while 
decreased expression during deprivation (Rao et al., 2007b). Likewise, increases in calcium dependent phospholipase 2 (cPLA2) and cyclooxygenase 2 (COX-2), enzymes controlling the release from the phospholipid membrane and metabolism of AA have been found following n3 PUFA deprivation (Rao et al., 2007a).

\subsubsection{Anti-Inflammatory Actions}

n-6 PUFAs are believed to be pro-inflammatory, while n-3 PUFAs appear to have antiinflammatory properties (Calder, 2009). These properties are thought to be related to metabolites rather than to the PUFAs themselves. The n-6 AA is metabolized into proinflammatory metabolites, including prostaglandins, leukotrienes and thromboxanes, while DHA and EPA are metabolized into anti-inflammatory metabolites, such as the D-resolvins (RvD1) and E-resolvins (RvE1 and RvE2), respectively. This metabolism takes place through a series of reactions involving 5-lipoxygenase. In addition, DHA can be metabolized by 15 lipoxygenase and subsequent enzymes to form neuroprotectin D1 (NPD1) (Hong et al., 2003). The resolvins and NPD1 have the ability to bring "resolution" to inflammation 1) by reducing TNF- $\alpha$ induced cytokine production (Serhan et al., 2002), 2) by regulating leukocyte and polymorphonuclear neutrophil (PMN) infiltration (Serhan et al., 2000) and 3) by increasing the ingestion of apoptotic PMN by macrophages (Schwab et al., 2007).

The n-3 PUFAs are thought to have two modes of action, one through the direct action of their metabolites - discussed above - and a second through their indirect effects on the n- 6 PUFAs (Serhan et al., 2008). The n-6 PUFA AA occupies the SN-2 position in the

phospholipid molecule, the same position as DHA. Increasing the amount of DHA in the 
phospholipid membrane thus competitively inhibits incorporation of AA in the membrane. Less AA would therefore be released by cPLA2, reducing the amount of pro-inflammatory metabolites. At the same time, of course, more DHA would be released by calcium independent phospholipase 2 (iPLA2), producing more of the anti-inflammatory NPD1, RvD1 and RvE's.

A second type of indirect inhibition of the $n-6$ system occurs because n- 3 and n- 6 PUFAs are metabolized by the same enzymes. When more n-3 PUFAs are present, DHA and EPA compete with AA for metabolic enzymes to produce more anti-inflammatory metabolites and less pro-inflammatory metabolites derived from AA. (Serhan et al., 2008).

\section{9 n-3 PUFAs and CNS disorders}

Recent studies have suggested that the n-3 PUFAs are related to a number different of central nervous system (CNS) functions and disorders. In most cases, the n-3 PUFAs are thought to have beneficial effects on these disorders. Since n-3 PUFAs affect so many CNS disorder, it gives some credence to the possibility that n-3 PUFAs may also have an effect on epilepsy, which is also a CNS disorder.

\subsubsection{Stroke}

There is evidence associating the n-3 PUFAs with stroke. Both negative and positive effects have been suggested. In terms of negative effects, the n-3 PUFAs are thought to be highly involved in ischemic reperfusion injuries. Ischemic injuries trigger the release of PUFAs 
from the phospholipid membrane (Bazan, 1970), and this release could lead to oxidative damage through lipid peroxidation.

On the other hand, the docosanoid metabolites derived from DHA have been shown to convey protection against ischemic strokes in a mouse model when administered intracerebroventricularly (i.c.v) (Marcheselli et al., 2003). NPD1 and DHA both reduce the activation of nuclear factor kappa-light-chain-enhancer of activated B cells (NFאB). They also reduce the expression of $\mathrm{COX}-2$, which is responsible for the metabolism of AA into proinflammatory metabolites (Marcheselli et al., 2003).

The injection of NPD1 in the $3^{\text {rd }}$ ventricle prior to the onset of stroke has been shown to reduce stroke volume in treated, as compared to vehicle injected, animals (Marcheselli et al., 2003). Further studies have shown that the i.v. infusion of DHA, with or without albumin, reduces the infract size and brain swelling in stroke models (Belayev et al., 2005; Belayev et al., 2009) . Interestingly, only the lower doses of DHA appeared to be effective in Belayev's studies. The higher doses did not appear to reduce infract size (Belayev et al., 2005; Belayev et al., 2009).

\subsubsection{Alzheimer's Disease}

n-3 PUFAs have also been associated with Alzheimer's disease (AD) (Jicha and Markesbery, 2010). An increased intake of n-3 PUFAs, for instance, has been associated with an increase in hippocampal volume in humans, which may be related to increased neurogenesis 
(Conklin et al., 2007). This would be beneficial as AD has been linked with a decrease in hippocampal volume (Barnes et al., 2005).

Likewise, the n-3 PUFAs may decrease the formation of beta amyloid fragments. The formation of amyloid-beta fragments is thought to be a cause of AD. These amyloid-beta (A $\beta$ ) fragments appear to be toxic to neurons, inhibiting synaptic transmission (Jicha and Markesbery, 2010). n-3 PUFAs are an integral part of the phospholipid membrane in the brain, in which the precursor for $\mathrm{A} \beta$, amyloid precursor protein, is found. Administration of DHA results in the formation of harmless fragments instead of the formation of the toxic $A \beta$ fragments (Jicha and Markesbery, 2010). In agreement with this, cell culture studies have demonstrated a reduction in the production in $\mathrm{A} \beta$ following treatment with DHA (Oksman et al., 2006).

\subsubsection{Depression}

Depression has also been linked to the n-3 PUFAs. In this case, the suggestion is the depression, like a number of neurological and psychological disorders, may result from underconsumption of n-3 PUFAs. Data have been drawn from a number of epidemiological studies which suggest a correlation between low omega-3 consumption and the risk of developing depressive syndromes (Freeman et al., 2006). DHA levels in erythrocyte membranes, for instance, are lower in depressive patients than in healthy patients (Edwards et al., 1998).

Several clinical trials have therefore attempted to look at the potential antidepressant properties of the n-3 PUFAs (Lin et al., 2010). These studies, however, have produced 
confusing results. While a few studies have reported beneficial effects of $n-3$ PUFAs supplementation on depression (Nemets et al., 2002; Peet and Horrobin, 2002), other studies have reported no effect (Marangell et al., 2003; Rogers et al., 2008). A recent meta-analysis has concluded that perhaps only EPA, and not DHA, has beneficial effects on depression, which has led to the mixed results reported in clinical studies (Martins, 2009).

While the results in clinical studies have been confused, animal studies have presented a clearer picture. The Porsolt forced swim test (FST) is a validated model of depression used to screen antidepressant drugs (Porsolt et al., 1977). The animal, usually a rat, is placed in a water bath with no possibility of escape. The latency for the animal to stop struggling and float passively is inversely correlated with the antidepressant effect of the drug (Porsolt et al., 1977).

DeMar and colleagues have reported that rat pups put on a diet deficient in n-3 PUFAs were more likely to display depressive like symptoms in the Porsolt forced swim test (FST). This was accompanied by a $36 \%$ reduction in DHA in the phospholipid membranes in the brains of the deprived animals, as compared to animals on adequate diets. In the brain membranes of deprived animals, DHA was replaced in large part by the n-6 PUFA DPA (DeMar et al., 2006b), which is an indicator of DHA deficiency (Galli et al., 1971).

In contrast, n-3 PUFA supplementation has been reported to have antidepressant effects in the FST in several animal studies (Carlezon et al., 2005; Lakhwani et al., 2007; Huang et al., 2008; Levant et al., 2008; Ferraz et al., 2010). The first study to investigate the antidepressant effects of the n-3 PUFAs was done by Carlezon and colleagues. Rats consuming approximately $0.72 \mathrm{~g} / \mathrm{kg} /$ day of $\mathrm{n}-3$ PUFAs had increased latencies to become immobile in the FST (Carlezon et al., 2005). 
This effect in the study of Carlezon et al. was only seen after one month of supplementation, however, while 3 and 10 days of supplementation were ineffective (Carlezon et al., 2005). A long latency for onset of action has also been reported by other groups, Venna et al., for instance reported that supplementation in mice only produced antidepressant effects in the FST following 5 weeks of treatment (Venna et al., 2009). Lakhwani et al. have similarly reported that acute administration of DHA 30 minutes prior to the FST failed to produce antidepressant effects (Lakhwani et al., 2007). Thus, orally administered n-3 PUFAs seem to take a month or more to produce antidepressant effects. These findings may also relate to our findings with dietary n-3 supplementation and seizure threshold (below).

The animal studies also suggest that the n-3 PUFAs may combine with other compounds to have their antidepressant effects. The suggestion is that the effects of supplementation with n-3 PUFAs may occur more rapidly in the presence of other compounds than with n-3 PUFAs alone. Carlezon et al., for instance, reported an additive effect between short duration supplementation of n-3 PUFAs (3 and 10 days) and a sub-therapeutic dose of uridine, which is an antidepressant in rats (Carlezon et al., 2005). Similar additive effects have been reported between DHA supplementation and fluoxetine (Lakhwani et al., 2007) and between n-3 PUFA supplementation and an ineffective dose of imipramine (Venna et al., 2009).

\subsubsection{Learning and Memory}

There have also been a few studies which have investigated the effects of n-3 PUFAs on learning and memory. The Morris water maze (MWM) is a model used to evaluate spatial 
memory in animals (Morris, 1984). The animal is place in a pool of cloudy water where it can escape onto a hidden platform. The animal must use spatial cues to find the platform. Over time the task is completed more and more quickly as the animal learns the cues (Morris, 1984).

The early studies mostly involved deprivation and showed that n-3 deprivation causes a decline in MWM performance. Rats on an n-3 PUFA deficient diet for three generations showed a reduction in working memory in the MWM as compared to animals reared on an adequate diet for 3 generation. The rats on the deficient diet had longer latencies for finding the hidden platform (Moriguchi et al., 2000). The deficient rats also showed an increase in brain AA and n-6 DPA and decrease in brain DHA (Moriguchi et al., 2000; Moriguchi and Salem, 2003).

This loss in brain DHA could be reversed in Moriguchi's studies if the pups were switched to a dam fed on an adequate diet. Weaning the animal onto an adequate diet also helped to partially restored brain DHA levels (Moriguchi and Salem, 2003). Deficient adult rats switched to an adequate diet, however, were not able to recover to the brain levels seen in rats raised on adequate diet (Moriguchi and Salem, 2003).

Lim and colleagues later concluded that it was specifically the loss of brain DHA, and not the non-specific loss of 22 carbon chain PUFAs, that resulted in the behavioural changes seen in the MWM during n-3 PUFA deprivation. n-6 DPA supplementation did not improve performance but resulted in the same working memory deficiency seen in DHA deficient animals (Lim et al., 2005).

Later studies showed that supplementation can also improve memory, just as deprivation can impair it. Supplementing animals with fish oil, containing both DHA and EPA, 
resulted in decreased escape latencies in the MWM as compared to animals on a normal adequate diet (Chung et al., 2008; Ferraz et al., 2010). This difference in escape latency was even more pronounced when supplemented animals were compared to animals on a n-3 PUFA deficient diet (Chung et al., 2008).

Genetic studies have also suggested a role for the n-3 PUFAs in learning and memory. The transgenic mouse known as the fat-1 mouse is an animal that has high levels of endogenous n-3 PUFAs. These mice express the C. elegans fat-1 gene, which allows the animal to produce n-3 PUFAs from n-6 PUFAs. Thus, the fat- 1 mice have an increase of $44 \%$ in brain DHA, which is was accompanied by increased cell proliferation in the hippocampus. They also perform better in the MWM, with reduced escape latencies as compared to the wild type mice (He et al., 2009).

The data from AD models further suggest a role for n-3 PUFAs. AD, of course, is associated with deficits in learning and memory. As noted in the section above, several studies have looked at n-3 PUFA supplementation in AD animal model, and they have shown that n-3 PUFA supplementation reduce $A \beta$ fragments in transgenic animals. In the same animals, low intake of n-3 PUFAs has been associated with a loss of spatial memory function and these affects are abolished by supplementation with n-3 PUFAs (Calon et al., 2004).

Hashimoto and colleagues have further demonstrated that an increase in n-3 PUFA intake protects against spatial memory loss in animals that are infused with A $\beta$ peptides. Infusion with $\mathrm{A} \beta$ peptides affects both reference and working memory, and is used as a model of AD (Hashimoto et al., 2005). 


\subsubsection{Anxiety}

The elevated plus maze is a standard test used to evaluate anxiety in animals. The test involves an elevated platform consisting of 4 arms in a "plus" shape. Two of the arms are "open" (with the floor visible to the animal), while the other two arms are "closed" (with walls enclosing the arms). The amount of time an animal spends on the open arms is inversely related to the amount of its anxiety. Compounds that increase anxiety will cause an animal to spend more time in the closed arms, while compounds with anxiolytic properties will cause an animal to spend more time in the open arms (Pellow et al., 1985).

Mice reared on an n-3 PUFA deficient diet spend significantly more time in the closed arms than mice reared on an adequate diet (Carrie et al., 2000; Takeuchi et al., 2003). This effect is reversed when the deficient animals are placed on a diet supplemented with DHA derived from either egg yolk or pig brain for 7 weeks (Carrie et al., 2000). Supplementation can act fairly quickly, since Takeuchi and colleagues have shown that one week of supplementation can reverse the effects by n-3 PUFA deprivation from birth (Takeuchi et al., 2003).

In related studies, the induction of "stress" by i.c.v. injection of corticotropin releasing hormone or interleukin $1 \beta$ has been shown to be attenuated by dietary supplementation with DHA (Takeuchi et al., 2003; Song et al., 2004). Similarly, Ferraz and colleagues have shown that fish oil supplementation results in a decrease in stress which is associated with a decrease in plasma corticosteroid (Ferraz et al., 2010).

There have been some negative studies in this field, however, which do not show anxiolytic effects of the n-3 PUFA supplementation (Frances et al., 1995; Moriguchi et al., 
2000). These contradictory results may be due to differences in the ways the plus maze tests were conducted. Many variables can change baseline anxiety/stress levels in animals, such as the amount of handling prior to the test or the amount of light present during the test (Fedorova and Salem, 2006).

\subsubsection{Aggression}

Only one study so far has looked at the effects of n-3 PUFAs on aggression in animal models. DeMar et al. have shown that depriving Long-Evans rats of n-3 PUFAs for 15 weeks results in increased aggression in the isolation-induced resident-intruder test. The deprived animals exhibited increased aggression as compared to control animals (DeMar et al., 2006b).

\subsection{0 n-3 PUFAs and the Heart}

There is a great deal of evidence relating n-3 PUFAs to improved heart function. Their effects in this field are very widely accepted.

The first evidence of the antiarrhythmic properties of n-3 PUFAs came from clinical observations of Greenland Inuit. Bang et al. found that the Inuit population of Greenland which had a lower incidence of ischemic heart disease - also had lower plasma triglycerides and cholesterol than a Danish control population (Bang et al., 1971). Moreover, it was found that the Inuit had higher n-3 PUFAs plasma concentrations and lower n-6 PUFAs concentrations (Dyerberg et al., 1975). These differences in plasma lipids - which were thought to relate to the lower incidence of cardiac disease - were also thought to relate to the 
higher intake of n-3 PUFAs in the Eskimo diet, as compared to the higher intake of n-6 PUFAs in the Danish diet (Bang et al., 1980).

Subsequently, the antiarrhythmic property of n-3 PUFAs was confirmed in animal studies. The acute injection of an n-3 PUFAs emulsion was found to successfully prevent the ventricular fibrillation induced by coronary ligation in dogs (Billman et al., 1994).

This in vivo study was followed by in vitro studies using cardiac myocytes (heart cells). n-3 PUFAs were found to alter cardiomyocyte contraction, reducing the contractions and protecting against the hyper-excitation induced by various drugs (Kang and Leaf, 1994). Both DHA and EPA, for instance, were found to reduce neonatal cardiomyocyte contractility, and to prevent high extracellular calcium and ouabain from increasing contractibility (Kang and Leaf, 1994). These effects were found to be modulated through a reduction of sodium currents (Xiao et al., 1995; Xiao et al., 1998). This reduction decreased membrane excitability and reduced the number of AP that occurred (Kang et al., 1995). These antiarrhythmics properties appeared to result from the direct binding of n-3 PUFAs to the VDSC (Kang and Leaf, 1996).

An interesting feature of these in vitro studies was that these antiarrhythmic properties of the n-3 PUFAs appeared to relate to the unesterified form of the n-3 PUFAs. The ethyl ester forms of EPA failed to reduce to the contractibility induced by ouabain (Kang and Leaf, 1994). The ethyl ester form is unable to be esterified in the phospholipid membrane. Although this does not completely rule out the possibility that they can be esterified, it suggests that the carboxylic group is needed to have action. Moreover, the ethyl ester form of EPA was also unable to bind to the VDSC (Kang and Leaf, 1996). 
Conversely, increasing the EPA and DHA concentration in the phospholipids alone does not convey any antiarrhythmic properties (Weylandt et al., 1996). Likewise, removing FFA with bovine serum albumin (BSA) - which would not affect esterified PUFAs eliminates the effects induced by the application of the fatty acids (Kang and Leaf, 1994; Xiao et al., 1995; Xiao et al., 1998).

\subsection{1 n-3 PUFAs and Epilepsy}

In the 1980s and 1990s, researchers began to investigate the possibility that the n-3 PUFAs might have antiepileptic as well as antiarrhythmic properties. This was first suggested by the fact that drugs that have antiarrhythmic properties - mediated through the VDSC - often also have anticonvulsive properties, suppressing hyperactivity in both the heart and the brain (Sanchez-Chapula and Josephson, 1983). Since the n-3 PUFAs were antiarrhythmic, it seemed possible that they might be anticonvulsant as well (Yehuda et al., 1994).

Subsequently, evidence to support this hypothesis was derived from in vitro, in vivo and clinical studies. These will be discussed below.

\subsubsection{In Vitro Evidence for the Anticonvulsant Effects of the n-3 PUFAs}

In in vitro studies, it has been shown that n-3 PUFAs reduce hippocampal hyperactivity by reducing sodium currents and increasing the inactivation time (Vreugdenhil et al., 1996). It has also been shown that n-3 PUFAs increase the stimulation intensity needed to cause AP firing, and reduce the number of APs that occur (Xiao and Li, 1999). 
Young et al. have similarly shown that the application of DHA to hippocampal slices reduces the repetitive neuronal firing and the amplitude of the spikes induced by bicuculline, a GABA-A antagonist (Young et al., 2000). The excessive firing caused by other proconvulsant molecules such as glutamate and PTZ is also reduced by the application of n-3 PUFAs (Xiao and $\mathrm{Li}, 1999)$. As in the in vitro studies in cardiomyocytes, these effects appear to be mediated by the unesterified form of the n-3 PUFAs, since the ethyl ester forms are ineffective (Xiao and Li, 1999).

\subsubsection{Evidence for the Anticonvulsant Effects of the n-3 PUFAs in Animal Studies}

Along with the in vitro studies, studies in intact animals have suggested that the n-3 PUFAs have anticonvulsant properties. A list of all of the animal studies is found in the literature appears in Table 1.1. There are contradictions in the whole animal data, however, as indicated below.

The first of the animal studies was published in 1981 by Climax and Sewell. This group found that i.c.v. injection of AA in ICI-GB1 mice provided seizure protection in the maximal pentylenetetrazole (PTZ) model (Climax and Sewell, 1981). It was, however, proconvulsant in the maximal electroshock (MES) model. The route of administration and seizure scoring were unconventional, and this early study was ignored.

It was not until 1994 that the next whole-animal experiment was published by Yehuda's group. These experimenters claimed that the chronic intraperitoneal (i.p.) administration of a mixture of LA and ALA in a ratio of 4:1 (a mixture known as the "SR-3 mixture") over a 
period of 3 weeks resulted in an increase in DHA in the brain and a large increase in seizure suppression in 4 different animal seizure models. This combination of ALA and LA in the SR3 mixture was thought to be optimal for the conversion of ALA to DHA (Yehuda et al., 1994; Yehuda et al., 1996; Rabinovitz et al., 2004).

In a later study, Voskuyl's group reported that an acute i.v. infusion of various fatty acids, including DHA and EPA, resulted in an increase in seizure threshold in a cortical stimulation seizure model in Wistar rats (Voskuyl et al., 1998). The infusion lasted for 30 minutes. Onset of seizure protection was not seen until 30 minutes had passed, and it lasted for 24 hours after the termination of the infusion.

Porta and colleagues performed the first dietary experiment in animals. They found that a diet supplemented with a mixture containing 70\% LA, 25\% ALA and 5\% OA and administered for 1 month resulted in an increase in PTZ seizure threshold in male Wistar rats. The increase in threshold in this study did not appear to be related to an increase in brain phospholipids, although increases in plasma EPA and ALA were observed (Porta et al., 2009).

A subsequent dietary study using EPA has failed to confirm these findings. Willis et al. have reported that that a diet in enriched in EPA for one month did not result in an increase in seizure threshold in any of 4 animal seizure models. They claim that this dietary manipulation raised n-3 PUFAs levels in the brain, but only n-3 DPA was found to be elevated while DHA and EPA were not (Willis et al., 2009). n-3 DPA, which is found in very small concentrations in the brain, has not yet been shown to be anticonvulsant in any model and it is possible that a rise in n-3 DPA would not convey anticonvulsant properties. 
At about the same time, Gilby and colleagues published a study in which Slow and Fast kindling rats were used (Gilby et al., 2009). The subjects consumed a diet enriched with DHA and EPA $(1000 \mathrm{mg} / \mathrm{kg} /$ day and $700 \mathrm{mg} / \mathrm{kg} /$ day for DHA and EPA respectively) for 70 days and were found to have higher electrical seizure thresholds following kindling. Curiously, the diet increased the rate of kindling in one strain of rat. This strain of rat, however, kindled slower than "normal" strains and supplementation actually brought the kindling rate back to normal (Gilby et al., 2009).

More recently, Pages et al. reported that chronic feeding of magnesium deficient diet supplemented with rapeseed (ALA rich diet) for 1 month resulted in seizure protection in $50 \%$ of Swiss mice in the magnesium deficiency dependent auditory seizure model. This diet, however, was not protective in 3 other seizure models; maximal PTZ, MES and NMDA seizure model (Pages et al., 2011). This could possibly be specific to the magnesium deficiency in the diet.

Musto and his group have recently published a study in which i.v. (Wistar rats, acutely before kindling), i.p. (C57BL/6, 4 days) and i.c.v. (C57BL/6 mice, 4 days) administration of DHA and NPD1 (with i.c.v. administration) resulted in a decrease in seizure severity, duration, and kindling rate in the hippocampus. Kindling resulted in an increase in production of endogenous NPD1. The group hypothesised that DHA conveys its anticonvulsant effects through its NPD1 metabolite (Musto et al., 2011).

Finally, a cross over veterinary trial in dogs with idiopathic epilepsy reported that 3 months supplementation did not result in a reduction in seizure frequency (Matthews et al., 2011). The seizure frequency of the subjects were low to start with, however, with 10/13 
Table 1.1: Evidence for the Anticonvulsant Effects of the n-3 PUFAs in Animal Studies

\begin{tabular}{|c|c|c|c|c|}
\hline Authors & Subjects & Model & Treatment & Results \\
\hline Climax et al. (1981) & $\begin{array}{l}\text { ICI-GB1 mice, } \\
18-24 \mathrm{~g}\end{array}$ & $\begin{array}{l}\text { Maximal PTZ } \\
(75 \mathrm{mg} / \mathrm{kg} \text { i.p.) } \\
\text { MES }(11 \mathrm{~mA}, 30 \mathrm{~Hz} \text {, } \\
\text { 30ms pulse width, } \\
0.2 \mathrm{~s} \text { duration) }\end{array}$ & $\begin{array}{l}\mathrm{AA}(5,10,25 \\
50 \mu \mathrm{g} / \mathrm{kg} \text { i.c.v })\end{array}$ & 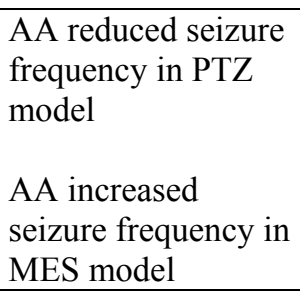 \\
\hline Yehuda et al. (1994) & $\begin{array}{l}\text { Male Sabra rats } \\
\text { (PTZ) and Sprague } \\
\text { Dawley rats }\left(\mathrm{FeCl}_{3} \text {, }\right. \\
\text { P-cresol), } 1 \text { month } \\
\text { old }\end{array}$ & $\begin{array}{l}\text { Maximal PTZ } \\
(50 \mathrm{mg} / \mathrm{kg}, \\
100 \mathrm{mg} / \mathrm{kg} \text { i.p. }) \\
\text { Repeated } \\
\text { subconvulsive PTZ } \\
(15 \mathrm{mg} / \mathrm{kg} \text { every } \\
15 \mathrm{~min} \text { until } \\
\text { induction of TC } \\
\text { seizure) } \\
\mathrm{FeCl}_{3}(5 \mu 1 \text { of } \\
100 \mathrm{mM} \text { i.c.v. in } \\
\text { amygdale }) \\
\text { P-cresol }(0.2 \mathrm{mg} / \mathrm{kg} \\
\text { i.p., } 7 \text { weeks prior to } \\
\text { treatment. Auditory } \\
\text { induced seizures by } \\
\text { buzzer } 5000 \mathrm{~Hz} \text { for } \\
10 \mathrm{~s}\end{array}$ & $\begin{array}{l}\text { ALA and AL in 1:4 } \\
\text { ratio ( } 40 \mathrm{mg} / \mathrm{kg} \text { i.p } \\
\text { for } 3 \text { weeks)(SR-3 } \\
\text { mixture) } \\
\text { Control treatment } \\
\text { received vehicle } \\
\text { (mineral oil) }\end{array}$ & $\begin{array}{l}\text { Increased seizure } \\
\text { suppression and } \\
\text { protection in all } 4 \\
\text { seizure models }\end{array}$ \\
\hline $\begin{array}{l}\text { Voskyul et al. } \\
\text { (1998) }\end{array}$ & $\begin{array}{l}\text { Male Wistar rats, } \\
175-200 \mathrm{~g}\end{array}$ & $\begin{array}{l}\text { Cortical electrical } \\
\text { stimulation }\end{array}$ & $\begin{array}{l}\text { DHA, EPA, LA, OA } \\
(40 \mu \mathrm{mol} \text { i.v. } \\
\text { infusion, } 30 \mathrm{~min}) \\
\text { Valproic acid } \\
\text { ( } 325 \mathrm{mg} / \mathrm{kg}, \text { i.v. } \\
\text { bolus) }\end{array}$ & $\begin{array}{l}\text { DHA and EPA } \\
\text { increased local and } \\
\text { generalized seizure } \\
\text { threshold } \\
\text { LA and OA had a } \\
\text { small increase in } \\
\text { seizure threshold } \\
\text { Seizure protection } \\
\text { 24hr after infusion }\end{array}$ \\
\hline $\begin{array}{l}\text { Lauritzen et al. } \\
(2000)\end{array}$ & $\begin{array}{l}\text { Male Wistar rats, } \\
250 \mathrm{~g}\end{array}$ & $\begin{array}{l}\text { Kainic acid } \\
\text { (10mg/kg i.p. } \\
30 \mathrm{~min} \text { after } \\
\text { treatment) } \\
\text { EEG recording in } \\
\text { CA1 }\end{array}$ & $\begin{array}{l}\text { ALA and PA (16 } \mu \mathrm{g} \\
\text { i.c.v, } 33 \mu \mathrm{g} / \mathrm{kg} \text { i.v.) }\end{array}$ & $\begin{array}{l}\text { ALA, but not PA, } \\
\text { protected against } \\
\text { Kainic acid seizures. } \\
\text { Less neuronal death } \\
\text { associated with } \\
\text { ALA }\end{array}$ \\
\hline $\begin{array}{l}\text { Blondeau et al. } \\
\text { (2002) }\end{array}$ & $\begin{array}{l}\text { Male Wistar rats, } \\
250-300 \mathrm{~g}\end{array}$ & $\begin{array}{l}\text { Kainic acid } \\
\text { ( } 7.5 \mathrm{mg} / \mathrm{kg} \text { i.p. } 3 \\
\text { days after treatment) }\end{array}$ & $\begin{array}{l}\text { DHA, AA, ALA, } \\
\text { PA (500nmol/kg } \\
\text { i.v.) }\end{array}$ & $\begin{array}{l}\text { ALA protected } \\
\text { against kainic acid } \\
\text { induced seizures }\end{array}$ \\
\hline
\end{tabular}




\begin{tabular}{|c|c|c|c|c|}
\hline Authors & Subjects & Model & Treatment & Results \\
\hline $\begin{array}{l}\text { Rabinovitz et al. } \\
\text { (2004) }\end{array}$ & $\begin{array}{l}\text { Male Long Evans, } \\
120-180 \mathrm{~g}\end{array}$ & $\begin{array}{l}\text { Maximal PTZ } \\
\text { (80mg/kg i.p. } \\
\text { following } 21 \text { days of } \\
\text { treatment) }\end{array}$ & $\begin{array}{l}\text { ALA and LA in 1:4 } \\
\text { ratio and } \\
\text { carbamazepine } \\
\text { ( } 40 \mathrm{mg} / \mathrm{kg} \text { i.p. for } 21 \\
\text { consecutive days) }\end{array}$ & $\begin{array}{l}\text { PUFAs resulted in } \\
\text { seizure suppression } \\
\text { Results similar to } \\
\text { carbamazepine }\end{array}$ \\
\hline Taha et al. (2006) & $\begin{array}{l}\text { Male Long Evans, } 1 \\
\text { month old }\end{array}$ & $\begin{array}{l}\text { Maximal PTZ } \\
\text { ( } 80 \mathrm{mg} / \mathrm{kg} \text { i.p. } \\
\text { following } 21 \text { days of } \\
\text { treatment) }\end{array}$ & $\begin{array}{l}\text { ALA and AL in 1:4 } \\
\text { ratio ( } 40 \mathrm{mg} / \mathrm{kg} \text { i.p } \\
\text { for } 3 \text { weeks) } \\
\text { Control treatment } \\
\text { received vehicle } \\
\text { (mineral oil) }\end{array}$ & $\begin{array}{l}\text { No effect of PUFAs } \\
\text { on seizure } \\
\text { protection }\end{array}$ \\
\hline Taha et al. (2008) & $\begin{array}{l}\text { Male fat-1 } \\
\text { transgenic mice and } \\
\text { wildtype mice, } 3 \\
\text { months old }\end{array}$ & $\begin{array}{l}\text { Maximal PTZ } \\
\text { (50mg/kg s.c.) }\end{array}$ & & $\begin{array}{l}\text { Seizure latency } \\
\text { delayed in fat-1 } \\
\text { mice } \\
\text { Latency correlated } \\
\text { with brain n-3 } \\
\text { profile }\end{array}$ \\
\hline Taha et al. (2008) & $\begin{array}{l}\text { Male Long Evans } \\
\text { rats, } 1 \text { month old }\end{array}$ & $\begin{array}{l}\text { Maximal PTZ } \\
\text { (80mg/kg i.p.) }\end{array}$ & $\begin{array}{l}\text { ALA and AL in 1:4 } \\
\text { ratio }(40,200,400, \\
1000 \mathrm{mg} / \mathrm{kg} \text { i.p for } 3 \\
\text { weeks) } \\
\text { Control treatment } \\
\text { received vehicle } \\
\text { (mineral oil) }\end{array}$ & $\begin{array}{l}40 \mathrm{mg} / \mathrm{kg} \text { still } \\
\text { ineffective } \\
200 \mathrm{mg} / \mathrm{kg} \text { protective } \\
\text { against PTZ seizures } \\
\text { Higher doses toxic }\end{array}$ \\
\hline Willis et al (2008) & $\begin{array}{l}\mathrm{C} 3 \mathrm{Heb} / \mathrm{FeJ} \text { and } \\
\mathrm{CD} 1 \text { mice, } 3 \text { weeks } \\
\text { old }\end{array}$ & $\begin{array}{l}\text { PTZ i.v. infusion } \\
\text { (CD1 mice, } \\
10 \mathrm{mg} / \mathrm{ml}, \\
150 \mu \mathrm{g} / \mathrm{min} \text { ) } \\
\text { Kainic acid (CD1 } \\
\text { mice, } 35 \mathrm{mg} / \mathrm{kg} \text { i.p.) } \\
\text { 6Hz (CD1 mice, } 3 \mathrm{~s} \\
\text { stimulation, } 0.2 \mathrm{~ms} \\
\text { pulse duration, } 6 \mathrm{~Hz} \text { ) } \\
\text { Fluorothyl model } \\
\text { (C3Heb/FeJ, } \\
10 \mu 1 / \mathrm{min} \text { drip) }\end{array}$ & $\begin{array}{l}\text { Chronic diet feeding } \\
\text { for } 1 \text { month. Each } \\
\text { diets were enriched } \\
\text { with different oils: } \\
\text { EPA oil } \\
\text { DHA oil } \\
\text { Vegetable oil }\end{array}$ & $\begin{array}{l}\text { No effect of PUFAs } \\
\text { on seizure } \\
\text { protection } \\
\text { No elevation in } \\
\text { brain DHA } \\
\text { following } 1 \text { month } \\
\text { of feeding }\end{array}$ \\
\hline Porta et al. (2008) & $\begin{array}{l}\text { Male Wistar rats, } 1 \\
\text { month old }\end{array}$ & $\begin{array}{l}\text { PTZ i.v. infusion } \\
(10 \mathrm{mg} / \mathrm{ml}, \\
333 \mu \mathrm{g} / \mathrm{min})\end{array}$ & $\begin{array}{l}\text { Chronic diet feeding } \\
\text { for } 1 \text { month: } \\
\text { Standard diet } \\
\text { ALA diet } \\
\text { KD diet }\end{array}$ & $\begin{array}{l}\text { ALA increased } \\
\text { seizure threshold }\end{array}$ \\
\hline
\end{tabular}




\begin{tabular}{|c|c|c|c|c|}
\hline Authors & Subjects & Model & Treatment & Results \\
\hline Gilby et al. (2009) & $\begin{array}{l}\text { Male Fast and Slow } \\
\text { kindling rats, } 4 \\
\text { months old }\end{array}$ & Amygdala kindling & $\begin{array}{l}\text { Fish oil diet vs. } \\
\text { Control diet }\end{array}$ & $\begin{array}{l}\text { Fish oil diet } \\
\text { increased } \\
\text { electrical seizure } \\
\text { threshold and } \\
\text { kindling rate in } \\
\text { slow kindling rats }\end{array}$ \\
\hline Taha et al (2010) & $\begin{array}{l}\text { Male Wistar rats, } 2 \\
\text { months old }\end{array}$ & $\begin{array}{l}\text { Maximal PTZ } \\
(105 \mathrm{mg} / \mathrm{kg} \text { i.p. })\end{array}$ & $\begin{array}{l}400 \mathrm{mg} / \mathrm{kg} \text { DHA s.c. } \\
(400 \mathrm{mg} / \mathrm{kg} \text { OA as } \\
\text { control) } \\
\text { PTZ testing at various } \\
\text { time point post DHA } \\
\text { injection }(15,30,60 \text {, } \\
120,240,480 \mathrm{~min})\end{array}$ & $\begin{array}{l}\text { DHA increased } \\
\text { seizure protection } \\
\text { at } 30 \mathrm{~min} \text { and } \\
60 \mathrm{~min} \\
\text { Effects loss at } \\
120 \mathrm{~min}\end{array}$ \\
\hline Musto et al. (2011) & $\begin{array}{l}\text { Male C57BL/6 mice, } \\
25-30 \mathrm{~g} \\
\text { Male Wistar rats, } \\
250-300 \mathrm{~g}\end{array}$ & $\begin{array}{l}\text { Rapid kindling } \\
\text { paradigm }\end{array}$ & $\begin{array}{l}\text { Femoral vein } \\
\text { cannulation }(12 \mathrm{mg} / \mathrm{kg} \\
\text { DHA, prior to } \\
\text { kindling, rats) } \\
\text { Alzet pump i.p. } \\
(1.68 \mu \mathrm{g} \text { DHA/day, } \\
\text { mice) } \\
\text { Alzet pump i.c.v. } \\
(50 \mathrm{nmol} \text { NPD1/day, } \\
\text { mice) }\end{array}$ & $\begin{array}{l}\text { DHA and NPD1 } \\
\text { reduced seizure } \\
\text { severity, duration, } \\
\text { and kindling rate } \\
\text { Seizures increased } \\
\text { production of } \\
\text { endogenous } \\
\text { NPD1 }\end{array}$ \\
\hline Pages et al. (2011) & $\begin{array}{l}\text { Female Swiss OF } \\
\text { mice, } 20-22 \mathrm{~g}\end{array}$ & $\begin{array}{l}\text { MES } \\
(4-7 \mathrm{~mA}, 50 \mathrm{~Hz}) \\
\text { Maximal PTZ } \\
\text { (LD100, s.c.) } \\
\text { NMDA induced } \\
\text { seizures } \\
\text { (LD100, s.c.) } \\
\text { Magnesium } \\
\text { deficiency } \\
\text { dependent auditory } \\
\text { seizure model } \\
\text { (MDDAS) }\end{array}$ & $\begin{array}{l}1 \text { month diet } \\
\text { treatment: } \\
\text { n-6 PUFA (+/- mg) } \\
\text { n-3 PUFA diet (+/- } \\
\text { mg) } \\
\text { MUFA diet (+/- diet) }\end{array}$ & $\begin{array}{l}\text { n-3 PUFAs (-mg) } \\
\text { diet was } \\
\text { protective in the } \\
\text { MDDAS model, } \\
\text { protecting } 50 \% \text { of } \\
\text { subjects } \\
\text { Magnesium } \\
\text { deficient diets had } \\
\text { no effects in the } \\
\text { other models }\end{array}$ \\
\hline $\begin{array}{l}\text { Matthews et al. } \\
\text { (2011) }\end{array}$ & $\begin{array}{l}15 \text { dogs, idiopathetic } \\
\text { epilepsy, on AEDs }\end{array}$ & & $\begin{array}{l}\text { 400mg EPA, 250 } \\
\text { DHA, 22mg Vit E } \\
\text { (per 10kg) } \\
\text { Blind, cross over trial } \\
3 \text { months }\end{array}$ & $\begin{array}{l}\text { No change in } \\
\text { frequency and } \\
\text { severity }\end{array}$ \\
\hline
\end{tabular}


animals having less than 1 seizure per month before the trial. During the trial, half the subjects had no seizures in both arms of the study. Of the animals to have been reported to have seizures $(n=6)$, a reduction in seizure frequency was seen in $66 \%$ of the subject. It is possible that significance might have been detected if the study had been conducted of a longer period of time and had included more subjects (Matthews et al., 2011).

\subsubsection{Clinical Evidence for the Anticonvulsant Effects of the n-3 PUFAs}

To date, there have been 5 clinical studies evaluating the anticonvulsant effects of n-3 PUFAs (Table 1.2). The first clinical trial that studied the anticonvulsant effects of n-3 PUFAs was published by Schlanger and colleagues in 2002. This was a small open study which involved epileptic patients with severe retardation. Patients, who were kept on their regular AEDs, were given a paste containing $65 \%$ of n-3 PUFAs for a period of 6 months, with each patient consuming approximately 2.3 and $0.9 \mathrm{~g}$ of DHA and EPA respectively per day. Although the final patient population was small $(n=5)$, the results were dramatic, with all 5 patients showing great improvements when n-3 PUFAs were added to their AEDs (Schlanger et al., 2002).

The next three published clinical studies reported essentially negative results (Yuen et al., 2005; Bromfield et al., 2008; DeGiorgio et al., 2008). Yuen et al. found transient anticonvulsant effects which were subsequently lost (Yuen et al., 2005). Bromfield and colleagues did not find any positive effects in the blinded period of the experiment, but it is interesting to note that seizure frequency decreased in 14 out of 19 patients in the open label 
Table 1.2: Clinical Studies Evaluating Anticonvulsant Effects of n-3 PUFAs

\begin{tabular}{|c|c|c|c|c|}
\hline Authors & Patients & Seizure types & Treatment & Results \\
\hline $\begin{array}{l}\text { Schlanger et al. } \\
(2002)\end{array}$ & $\begin{array}{l}5 \text { patients, open label } \\
\text { Profound mental } \\
\text { retardation with } \\
\text { epilepsy }\end{array}$ & $\begin{array}{l}\text { tonic-clonic } \\
1 \text { patient also had } \\
\text { absence seizures }\end{array}$ & $\begin{array}{l}2.3 \mathrm{~g} \text { DHA, } 0.9 \mathrm{~g} \\
\text { EPA daily } \\
6 \text { months }\end{array}$ & $\begin{array}{l}\text { Reduced seizure } \\
\text { frequency in all } \\
\text { patients }\end{array}$ \\
\hline Yuen et al. (2005) & $\begin{array}{l}57 \text { patients, double } \\
\text { blind placebo control } \\
35 \text { males, } 22 \text { females } \\
19-65 y r \text { old }\end{array}$ & $\begin{array}{l}2 \text { generalized } \\
51 \text { focal } \\
4 \text { other }\end{array}$ & $\begin{array}{l}\text { 1g EPA, } 0.7 \mathrm{~g} \text { DHA } \\
\text { daily } \\
12 \text { week blind, } 12 \\
\text { week open label }\end{array}$ & $\begin{array}{l}\text { Reduction in } \\
\text { seizure frequency } \\
\text { in first } 6 \text { weeks } \\
\text { Effects loss in } \\
\text { next } 6 \text { weeks } \\
\text { Few adverse } \\
\text { effects: sleepiness } \\
\text { and diarrhea }\end{array}$ \\
\hline $\begin{array}{l}\text { Bromfield et al. } \\
\text { (2008) }\end{array}$ & $\begin{array}{l}21 \text { patients, } \\
\text { randomized placebo } \\
\text { controlled trial } \\
12 \text { males, } 9 \text { females } \\
\text { 22-62yr old } \\
\text { Intractable seizures (4 } \\
\text { or more per month) }\end{array}$ & $\begin{array}{l}16 \text { focal } \\
2 \text { generalized } \\
3 \text { symptomatic }\end{array}$ & $\begin{array}{l}0.66 \mathrm{~g} \text { EPA and } \\
0.22 \mathrm{~g} \text { DHA twice } \\
\text { daily } \\
12 \text { weeks blind, } 4 \\
\text { weeks open label } \\
\text { (optional) }\end{array}$ & $\begin{array}{l}\text { No effect of } \\
\text { treatment during } \\
\text { blind period } \\
\text { Significant } \\
\text { positive effect of } \\
\text { treatment during } \\
\text { open label }\end{array}$ \\
\hline $\begin{array}{l}\text { DeGiorgio et al. } \\
\text { (2008) }\end{array}$ & $\begin{array}{l}11 \text { patients, double } \\
\text { blind cross over } \\
\text { Intractable seizures ( } 3 \\
\text { or more seizures per } \\
\text { month) }\end{array}$ & N/A & $\begin{array}{l}1.73 \mathrm{~g} \text { EPA, } 1.15 \mathrm{~g} \\
\text { DHA per day } \\
12 \text { weeks } \\
\text { supplementation, } 6 \\
\text { weeks wash out, } 12 \\
\text { weeks placebo }\end{array}$ & $\begin{array}{l}\text { Seizure frequency } \\
\text { increased in both } \\
\text { fish oil and } \\
\text { placebo group }\end{array}$ \\
\hline $\begin{array}{l}\text { Al Khayat et al. } \\
\text { (2010) }\end{array}$ & $\begin{array}{l}20 \text { patients, double } \\
\text { blind placebo control } \\
\text { Children (3-10yr old) } \\
\text { Intractable seizure } \\
\text { (one seizure per } \\
\text { month for } 18 \text { months) }\end{array}$ & $\begin{array}{l}10 \text { generalized } \\
10 \text { focal }\end{array}$ & $\begin{array}{l}\text { 700mg DHA, 300mg } \\
\text { EPA per day } \\
6 \text { months } \\
\text { supplementation }\end{array}$ & $\begin{array}{l}\text { Negative } \\
\text { correlation } \\
\text { between DHA } \\
\text { and EPA plasma } \\
\text { concentration } \\
\text { following } \\
\text { supplementation } \\
\text { and seizure } \\
\text { duration and } \\
\text { severity }\end{array}$ \\
\hline
\end{tabular}


period of the study, which involved a longer period of administration (Bromfield et al., 2008). DeGiorgio et al actually reported increased seizure frequency (DeGiorgio et al., 2008).

It is important to note, however, all three of these negative studies, used lower doses of n-3 PUFAs and a shorter duration of administration as compared to the study published by Schlanger et al. Recently, a new, double-blind study has been published by Al Khayat et al. showing anticonvulsant effects of a DHA-enriched diet in children with intractable epilepsy (Al Khayat et al., 2010). This is the first study to use a duration of administration similar to Schlanger et al., since the duration was 6 months. This group found that patients with intractable epilepsy had lower plasma levels of DHA before the diet, as compared to healthy controls. Supplementation with DHA elevated DHA plasma levels, and this was negatively correlated with seizure severity and duration.

\subsubsection{Recent Work at the University of Toronto}

Work in our own group at the University of Toronto began with an attempt to replicate SR-3 mixture study done by Yehuda and his group (Yehuda et al., 1994). We were not able to replicate Yehuda's work. Our group failed to find an anticonvulsant effect of $40 \mathrm{mg} / \mathrm{kg}$ of SR-3 mixture administered i.p. for 21 days (Taha et al., 2006). The seizure model used in both of these studies was the maximal PTZ model, an animal model of tonic-clonic seizures (Fisher, 1989).

A colleague in the Department of Nutritional Sciences brought to our attention, however, that the administration of $40 \mathrm{mg} / \mathrm{kg}$ of the SR-3 mixture only increased a rat's daily intake of the two fatty acids in the SR-3 mixture (ALA and LA) by $1.2 \%$. It seemed unlikely 
that such a small increase could generate anticonvulsant effects. Therefore the dose of 40 $\mathrm{mg} / \mathrm{kg}$ of the SR-3 mixture was compared to a higher dose of $200 \mathrm{mg} / \mathrm{kg}$ in the maximal PTZ model. It was once again confirmed that $40 \mathrm{mg} / \mathrm{kg}$ was not anticonvulsant. The dose of $200 \mathrm{mg} / \mathrm{kg}$, however, significantly increased seizure latency in the maximal PTZ model (Taha et al., 2009a). This increase in seizure latency appeared to be correlated with an increase in the unesterified pool of n-3 PUFAs in the brain (Taha et al., 2009a). This apparent increase in the brain, however, must be viewed with some caution since brain fatty acid analysis was done after seizures had been elicited in the animal. Seizures release fatty acids, including n-3 PUFAs, from the phospholipid membrane (Bazan, 1971). It should be noted in this study, that n-3 PUFAs caused an increase in latency to seizure onset, but not the complete suppression of seizures that Yehuda had reported.

In the next study in our laboratory, the chronic dietary effects of the n-3 PUFAs were evaluated. Rats were put on a diet, in which $20 \%$ of the fat content came from fish oil, which is high in EPA and DHA (Taha, 2009). The fish oil subjects were compared to control subjects on a standard diet. Electrical seizure thresholds were measured every month via chronically implanted electrodes. The cortical seizure threshold rose in the fish oil subjects following 3 months of chronic feeding. No elevation in seizure threshold was seen in amygdala, however, after 3 months. The fish oil content of the diet was then raised to $40 \%$ of the fat content for the amygdala-implanted subjects and the diet was continued. Amygdala seizure thresholds rose about 2 months after the start of the $40 \%$ diet, or after about 5 months after the start of the original diet (Taha, 2009).

In an interesting follow-up to the diet experiment, seizure thresholds were measured in subjects on a normal diet or a diet deficient in n-3 PUFAs. Animals deficient in n-3 PUFAs 
were found to have reduced seizure thresholds in both the amygdala and the cortex when compared to animals on adequate diets (Taha et al. unpublished results). These changes were seen after approximately 4 months of feeding.

A further study in our laboratory involved the fat-1 mouse, which endogenously produces higher DHA levels. Fat-1 mice were compared to wild type mice in the maximal PTZ test. The fat- 1 mice showed a $45 \%$ increase in seizure latency, but this was not significant due to variability (Taha et al., 2008). When the brains were assayed, however, it was found that there was a great deal of variability in the brain DHA levels. A subsequent correlation analysis detected a correlation between the seizure latency and brain DHA levels (Taha et al., 2008), suggesting that higher levels of brain DHA was protective against seizures.

Our group next decided to look at the acute effects of DHA in the maximal PTZ model. As noted above, Voskyul et al. had demonstrated anticonvulsant effects of both DHA and EPA in studies involving acute infusions (Voskuyl et al., 1998). We therefore tested the acute administration of DHA dissolved with albumin against maximal PTZ seizures. A dose of $400 \mathrm{mg} / \mathrm{kg}$ was injected s.c. and PTZ was injected i.p. at various timepoints. A significant increase in latency to seizure onset was seen 30 and 60 minutes following DHA injection, although no actual seizure suppression occurred (Taha et al., 2010).

The s.c. study (described above), plus a related pilot study (described below), showed acute effects of DHA. These acute studies, however, raised several questions which required further consideration, and which are the subject of experiments in this thesis. The first question relates to the slow time of onset of DHA's actions. In our s.c experiment, DHA started to show anticonvulsant effects only at 30 minutes following s.c. injection, with maximal effects 
being seen at 1 hour. Other high-partition coefficient compounds in our laboratory have acted much more quickly after s.c. injection (Lonsdale and Burnham, 2003).

A second question relates to why DHA was active for such a long time. The anticonvulsant effects in our s.c. experiment lasted from 30 to 60 minutes and disappeared between 60 minutes and 120 minutes after injection (Taha et al., 2010). This is in disagreement with the fact that unesterified DHA has a half life of approximately 30 seconds in the plasma (Robinson et al., 1992). If DHA's anticonvulsant effects relate to the unesterified pool in the plasma, then short duration of action should be expected as DHA in the unesterified pool would have been back to baseline levels within 2.5 minutes following injection. If DHA's anticonvulsant effects relate to the unesterified DHA that leaves the plasma and enters the brain phospholipids, then the effects should also be short, since that pool is quickly esterified, and the esterification is complete within 40 seconds (Chen et al., 2009). Finally, if DHA's anticonvulsant effects relate to the DHA that enters the brain and is esterified and released, then they should be much longer, as the half life of esterified DHA in the brain is approximately 33 days (DeMar et al., 2004). Thus, the s.c. experiment raised a question or questions about the time course of DHA's actions.

A third question relates to a pilot s.c. dose-response study that was performed to evaluate which dose to use in future experiments. In this study, which only involved a few rats, it was noticed that DHA appeared to produce an inverted-U shape dose response curve. Maximal effects were seen at $400 \mathrm{mg} / \mathrm{kg}$, but the effects were lost at higher doses (Taha et al., 2010). Inverted-U dose response curves are not common in pharmacology. 
In the final study that preceded this thesis, DHA release from the brain was tested. It has been known for some time that brain insults, including brain hyperactivity, caused the release of lipids from the phospholipid membrane (Bazan, 1970, 1971; Bazan et al., 1981). While AA has been the main focus of these release studies, it has also been noted that DHA is also released from the membrane following brain hyperactivity. Our group, therefore, examined DHA and AA release before and after PTZ seizures. Experimental subjects were pretreated with DHA or AA, while control subjects were not. We hypothesized that pre-treatment with s.c. DHA might cause an increase in release in DHA from the phospholipid pool, either before or during seizures, into the unesterified pool, which might raise seizure thresholds. Contrary to our hypothesis, pre-treatment with an acute injection of DHA did not result in increased release of DHA from the phospholipid membranes either before or after a seizure (Taha, 2009).

\subsection{Unanswered Questions - Goals of This Thesis}

Work from our own and other laboratories suggests that n-3 PUFAs could potentially be used for anticonvulsant therapy. Acute, semi-chronic, and chronic administration of n-3 PUFAs have all been shown to increase seizure thresholds in both PTZ and electrically triggered seizures. On the other hand, n-3 PUFA deprivation, which reduces brain PUFAs, has been shown to reduce seizure threshold (Taha et al. in preparation). In our hands, acute administration of pure DHA has raised seizure thresholds, suggesting that DHA may be a compound of particular use in anticonvulsant therapy. 
The existing data, however - and particularly the data from our laboratory - have raised several questions that need to be answered. These will be addressed in this thesis. They can be divided into questions related to chronic (dietary), sub-chronic and acute studies.

\section{Questions Related to Chronic (Dietary) Studies}

\section{1) Dietary Experiments - Would the oral administration of fish oil have had more rapid effects on the amygdala if a higher dose had been administered from the onset? Why does oral administration of n-3 PUFAs take so long to have an effect?}

Higher Dose: In our first oral administration studies (Taha, 2009) the cortical seizure threshold rose approximately 3 months following the start of fish oil supplementation, but the amygdala threshold did not rise at that time. Subsequently, a higher dose of fish oil was administered, and then the amygdala threshold rose (Taha, 2009). Was the rise in amygdala threshold caused by total duration of treatment ( 5 months) or by the increased dose of fish oil? If a higher dose of fish oil were administered from the start, would amygdala thresholds rise more quickly?

To answer this question, we have therefore applied the higher dose of fish oil from the start in a new group of amygdala implanted subjects. We hypothesized that a long duration of supplementation would be needed to raise amygdala threshold.

\section{Questions Related to Sub-chronic Studies}

\section{2) Can Sub-Chronic Injections of DHA Raise Thresholds?}

Our work in the PUFA field began with an attempt to replicate Yehuda's sub-chronic study involving short-chain PUFAs. This involved administering LA and ALA in a ratio of 4:1 
for 21 days i.p. Yehuda and colleagues had reported dramatic results with this paradigm (Yehuda et al., 1996; Rabinovitz et al., 2004), while our group found only subtle effects which occurred only at higher doses (Yehuda et al., 1994; Rabinovitz et al., 2004; Taha et al., 2009a).

During the course of our work, it was indicated to us that Bruzzese (2005) has claimed dramatic effects - like those claimed by Yehuda - after injecting rats for 14 days i.p. with pure DHA or DHA ethyl ester (Bruzzese 2005).

We therefore attempted to replicate the experiment of Bruzzese. We hypothesized that sub-chronic administration of DHA would result in anticonvulsant effects, like the administration of the SR-3 mixture. However, due to previous attempts to replicate Yehuda and colleagues, we did not believe we would achieve actual seizure suppression as reported by Bruzzese.

\section{Questions Related to Acute Studies}

The majority of our unanswered questions related to the acute effects of DHA:

\section{3) Is the inverted-U dose-response curve real or only an artefact?}

True inverted-U dose response curves are rare in pharmacology. They have been reported in studies with hormones, where hormones act on multiple receptors which can antagonize act each other at high doses (Diamond et al., 1992). With DHA, however, the apparent inverted-U relationship may simply reflect the possibility that DHA may be coming

out of solution at higher doses. Phenytoin, another compound with poor water solubility, crystallizes when injected intramuscularly (i.m.) (Serrano and Wilder, 1974) and also appears to come out of solution when injected i.p. (McNamara et al., 1989). 
We hypothesized that DHA might be coming out of solution at higher doses. We first tried to confirm the original finding of an inverted-U s.c. dose-response curve. A s.c. doseresponse curve with more subjects was constructed using the maximal PTZ model. We then attempted to test the hypothesis that DHA was coming out of solution at higher doses. Blood levels of DHA were measure at lower (effective) and higher (ineffective) doses after s.c. injection. We expected to see higher blood levels at the lower (effective) dose than at the higher (ineffective) dose.

\section{4) Why does acute administration of DHA take 30 minute to produce anticonvulsant effects, and why are the effects lost after 2 hours?}

DHA is a compound with a high partition coefficient, which increases absorption, and a short half life, which should lead to a short duration of therapeutic action (Robinson et al., 1992). These pharmacokinetics are not in agreement with the data previously obtained in our laboratory.

In terms of absorption, other compounds with high partition coefficients have been found to be active in less than 5 minutes after s.c. injection (Lonsdale and Burnham, 2003). We would expect a quick onset of DHA's actions. In our past studies, however, but we have seen no effects before 30 minutes after s.c. (Taha et al., 2010).

In terms of elimination, plasma unesterified DHA has a half-life of approximately 30 seconds (Robinson et al., 1992). Therefore, if the unesterified form of DHA is the active form, 
it would be expected that DHA's effects would disappear shortly after injection. In our hands, however, DHA's effects have been seen to last from 1 to 2 hours (Taha et al., 2010).

DHA may need to be incorporated into the phospholipid membrane in order to have effect. However, the half life of DHA within the phospholipid membrane is 33 days approximately (DeMar et al., 2004). This is not what we found, since DHA's effects disappeared by 2 hours.

We therefore hypothesized that the s.c. administration of DHA acted as a slow release preparation - allowing unesterified DHA to slowly enter the blood stream from the site of injection - and providing a relatively slow onset of action and a relatively long duration of action.

5) If the inverted-U is an artefact, could administering DHA by the i.v. route produce more normal dose-response and time-response curves?

McNamara et al. showed that phenytoin comes out of solution when injected i.p., but is powerfully anticonvulsant when administered i.v. (McNamara et al., 1989). Therefore it seemed possible that DHA might produce more understandable effects if administer via the i.v. route, which has simpler pharmacokinetics.

To test this hypothesis, DHA was given i.v. Dose-response and time-response curves were constructed using this route. 
CHAPTER 2

General Methods 


\subsection{Subjects}

Male Wistar rats served as subjects in all experiments. Rats were obtained from Charles River Canada (La Prairie, Qc) and were received at the animal care facility at approximately 1 month of age, except in the infusion experiments, where rats arrived weighing approximately $350 \mathrm{~g}$.

Rats were individually housed in all experiments in 24 X 24 X 45cm transparent plastic cages with corn cob bedding and environmental enrichment. Water and rat chow (Teklad Global) was available ad libitum. A $12 \mathrm{hr}$ light/dark cycle was maintained in the vivarium (lights on at $7 \mathrm{am}$ ) and a constant temperature of $21^{\circ} \mathrm{C}$.

Subjects were allowed to acclimatise to the vivarium for a minimum of 1 week prior to any testing. They were also handled for a minimum of 5 days during the acclimatisation period to minimize stress.

\subsection{Drugs and Preparation of Drug Solutions}

DHA, DHA ethyl ester (DHA EE), AA, OA were purchased from Nu-Chek Prep (Elysian, MN). The vehicle additive, bovine serum albumin (BSA), was purchased from Sigma Aldridge (St. Louis. MO). Drug solutions were prepared at a ratio of $1.43 \mathrm{mg}, 1.42 \mathrm{mg}, 1.43 \mathrm{mg}$, and $1.38 \mathrm{mg}$ to $1 \mathrm{mg}$ of BSA for DHA, DHA EE, AA, and OA, respectively. All compounds were dissolved in $0.9 \%$ saline. Solutions were kept on ice during injection and kept at $-80^{\circ} \mathrm{C}$ overnight. 
The GABA-A antagonist PTZ was purchased from Sigma Aldridge (St. Louis, MO). It was prepared by adding 50mg of PTZ to each $\mathrm{ml}$ of physiological saline.

\subsection{Procedure for Maximal PTZ Testing}

In Chapters 4,5,7 and 8, animals were seizure tested with PTZ. Prior to testing, a titration was done to determine the dose to administer to ensure all animals seized. Since we had found the response to PTZ was variable, pilot studies were performed on each batch of rats to determine the PTZ dose that would be used in seizure tests. Optimal doses ranged from $100 \mathrm{mg} / \mathrm{kg}$ to $110 \mathrm{mg} / \mathrm{kg}$.

On the day of testing, PTZ was administered via i.p. injection. Once injected, the animal was placed in an open field and observed for a period of $15 \mathrm{~min}$, or until tonic-clonic seizure occurred. Seizure occurrence and latency to tonic forelimb extension were measured.

\subsection{Procedure for Cannulation}

In i.v. experiments (Chapters 6 and 7), subjects were cannulated in one or both tail veins. Subjects were brought to the testing room 1 hour before cannulation to allow for acclimatisation. Their tails were then placed under heat lamp for $20 \mathrm{~min}$ to cause vasodilation of the tail veins. Subjects were then loosely restrained with a handling towel and a 24 gauge angiocath (Becton Dickson, Franklin Lake, NJ) was placed within the tail vein and held in place with surgical tape. 


\subsection{Euthanasia}

Several methods of euthanasia were used in different experiments. In Chapter 6-8, animals were euthanized with an overdose of T-61 $(100 \mathrm{mg} / \mathrm{kg})$ through the angiocath already secured in the tail vein. In Chapter 4, seizing animals were euthanized with an intra-cardiac overdose of T-61 (100mg/kg) while non-seizing animals were euthanized by $\mathrm{CO}_{2}$ asphyxiation. In Chapter 3, animals were euthanized at the end of the study by $\mathrm{CO}_{2}$ asphyxiation. In Chapter 5 , animals were euthanized by an overdose of pentobarbital (Somnitol ${ }^{\circledR} 100 \mathrm{mg} / \mathrm{kg}$ ) delivered i.p.

2.6 Serum Phospholipid, Triglyceride, Cholesteryl Ester and Unesterified Free Fatty Acid Isolation

Total lipids were extracted from $200 \mu \mathrm{l}$ of serum into $6 \mathrm{ml}$ of chloroform / methanol $(2: 1 \mathrm{v} / \mathrm{v})$, using $2 \mathrm{ml}$ of $0.88 \% \mathrm{KCl}$ to separate the aqueous phase, followed by a wash with 4 $\mathrm{ml}$ of chloroform (Folch et al., 1957). The total lipid extract was then dried under nitrogen and reconstituted in $100 \mu \mathrm{l}$ of chloroform.

Thin layer chromatography (TLC) was used to separate phospholipids, FFA, triglycerides and cholesteryl esters from the total lipid extracts. TLC plates (Whatman LK6D plates, precoated with $250 \mu \mathrm{m}$ of Silica Gel 60A) were washed in chloroform and methanol (2:1) and activated by heating at $100^{\circ} \mathrm{C}$ for 1 hour prior to use. The total lipid extract $(100 \mu \mathrm{l})$ was loaded onto the TLC plates and the bands were resolved in heptane: diethyl ether: glacial acetic acid (60:40:2 by volume), alongside authentic standards (Avanti, Alabaster, AL). The 
plates were sprayed with $0.1 \%(\mathrm{w} / \mathrm{v})$ 8-anilino-1-naphthalenesulfonic acid, and the bands corresponding to the phospholipid, FFA, triglyceride and cholesteryl ester standards were identified under ultraviolet light. The bands were scraped off the plates into $15 \mathrm{ml}$ glass screw cap tubes with Teflon lined caps. Non-esterified heptadecanoic acid (Sigma, St. Louis, Mo) in hexane was added as an internal standard to each lipid fraction. The lipid fractions were then methylated in $14 \%$ methanolic $\mathrm{BF}_{3}(2 \mathrm{ml})$ and hexane $(2 \mathrm{ml})$ at $100^{\circ} \mathrm{C}$ for 1 hour. The samples were allowed to cool at room temperature for 10 minutes and centrifuged at $1460 \mathrm{rpm}$ for 10 minutes following the addition of deionized water $(2 \mathrm{ml})$. The upper hexane layer was extracted, dried under nitrogen and reconstituted in 60-100ul hexane for fatty acid methyl ester (FAME) analysis of phospholipids, free fatty acids, triglycerides and cholesteryl esters.

\subsection{Fatty Acid Methyl Ester (FAME) Analysis by Gas-Chromatography}

FAMEs were analyzed on a Varian-430 gas chromatograph (Varian, Lake Forest, CA, USA) equipped with a Varian FactorFour capillary column (VF-23ms; $30 \mathrm{~m}$ x $0.25 \mathrm{~mm}$ i.d. $\mathrm{x}$ $0.25 \mu \mathrm{m}$ film thickness). One $\mu \mathrm{l}$ of FAMEs was injected in splitless mode. The carrier gas was helium, set to a constant flow rate of $0.7 \mathrm{ml} / \mathrm{min}$. The injector and detector ports were set at $250^{\circ} \mathrm{C}$. FAMEs were eluted using a temperature program set initially at $50^{\circ} \mathrm{C}$ for $2 \mathrm{~min}$, followed by a ramp-up at $20^{\circ} \mathrm{C} / \mathrm{min}$ to $170^{\circ} \mathrm{C}$, a hold at $170^{\circ} \mathrm{C}$ for $1 \mathrm{~min}$, and an increase of $3^{\circ} \mathrm{C} / \mathrm{min}$ to $212^{\circ} \mathrm{C}$ and a hold at $212^{\circ} \mathrm{C}$ for $5 \mathrm{~min}$. Peaks were confirmed by identifying the retention times of authentic FAME standards of known composition (Nu-Chek Prep, Elysian, $\mathrm{MN})$. Fatty acid concentrations ( $\mu \mathrm{g} / \mathrm{ml}$ of serum) were calculated by proportional comparisons of the gas chromatography peak areas with that of the heptadecanoic acid internal standard. 


\section{CHAPTER 3}

Anticonvulsant Effects of Chronic Fish Oil Administration in the Electrical Afterdischarge Threshold Model in the Amygdala 


\subsection{Rationale}

Experiment 3 was designed to evaluate the effects of chronic n-3 PUFA administration - in the form of dietary fish oil supplementation - on electrical seizure thresholds in the amygdala.

Previous work in our laboratory had demonstrated that chronic fish oil feeding elevated electrical seizure threshold in both the cortex and the amygdala (Taha, 2009). The amygdala thresholds, however, took 5 months to rise. Moreover, prior to the elevation in the amygdala seizure threshold, the fish oil content was increased from $20 \%$ of total fat to $40 \%$ of total fat in the diet.

This change in dietary fat content raises the question of whether the elevation in electrical thresholds in the amygdala would have appeared more rapidly if a higher fish oil content had been used from the beginning of the study. Experiment 3 was designed to confirm the previous finding that dietary fish oil would raise amygdala seizure thresholds and to address the question of whether these changes would appear more quickly if a higher content of fish oil was used from the start of the diet. A diet enriched with $40 \%$ of fish oil was employed from the start Experiment 3.

\subsection{Methods}

The full experimental paradigm for Experiment 3 is illustrated in figure 3.1. 
Figure 3.1. Study Design

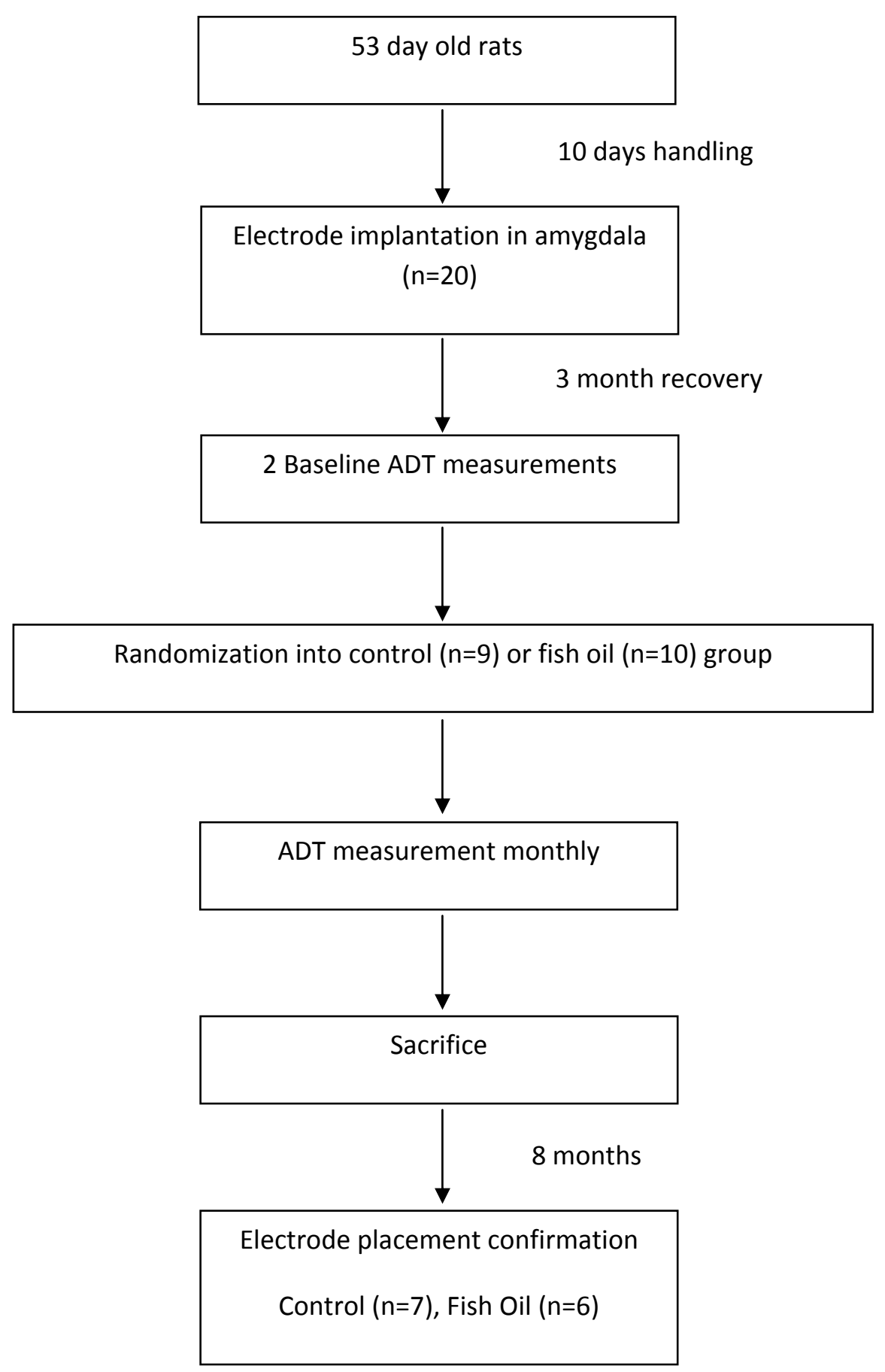




\subsubsection{Subjects}

Male 53 day-old Wistar rats were obtained from Charles River (La Prairie, Qc) and housed as described in the General Methods section. Initially, they were fed a diet of regular rat chow (Teklad Global). The study started with 20 animals. After removing subjects which were not properly implanted in the amygdale at the end of the experiment, the control and fish oil group had 7 and 6 subjects respectively.

\subsubsection{Procedure for Surgery}

Approximately 10 days after arrival at the animal facility, an electrode was implanted in the right amygdala of each subject. $(n=20)$. Anesthesia was induced with $5 \%$ isofluorane prior to surgery and subjects were maintained anesthetized with 2-3\% isofluorane during surgery. Stainless steel bipolar electrodes (MS303/1, Plastics One, Roanoke, VA, USA) were then implanted in the right basolateral amygdala using standard stereotaxic procedure (Pellegrino and Cushman, 1971). The amygdala coordinates were as follows (mm): anteriorposterior, -2.8; medial-lateral, 4.6; and dorsal-ventral, -8.6 (skull surface). The incisor bar was set so that bregma and lambda were in a horizontal plane. The electrodes were fixed to the skull with 3 to 4 stainless steel anchor screws and acrylic, dental cement (Nuweld, LD, Caulk). All subjects received buprenorphine $(0.05 \mathrm{mg} / \mathrm{kg} \mathrm{s.c.})$ as an analgesic and physiological saline (1 ml/kg s.c.) for rehydration immediately after surgery. Subjects were allowed approximately 2-3 months for recovery before baseline afterdischarge threshold (ADT) measurements were done. 


\subsubsection{ADT measurements in the Amygdala}

Approximately 2-3 months following electrode placements, baseline ADTs in the amygdala were measured using the ascending series technique (Pinel et al., 1976). The stimulus consisted of a 1 second train of $60 \mathrm{~Hz}$ biphasic square-wave pulses with a $1 \mathrm{~ms}$ positive and a $1 \mathrm{~ms}$ negative peak. The stimulus was generated by a Grass model S- 88 stimulator (Grass Instruments, Quincy, MA, USA). Electroencephalographic (EEG) activity at the stimulated focus was recorded on a Grass model 6 electroencephalograph (Grass Instruments, Quincy, MA, USA), using a device that switched the electrode circuit to the electroencephalograph immediately following stimulation. Afterdischarges were digitally recorded using an IBM compatible personal computer with a National Instruments digital acquisition board (AT-MIO16E) and the National Instruments Labview 7.0 graphical programming environment. ATD measurement was started at $40 \mu \mathrm{A}$ (peak to peak) and increased in $20 \mu \mathrm{A}$ increments every 5 minutes until an afterdischarge was detected in the EEG. Behavioural seizures (when they occurred) were scored using the Racine scale. The Racine scale is divided into 5 seizure stages: 1) jaw clonus, 2) head nodding (head clonus), 3) forelimb clonus, 4) rearing, and 5) rearing and falling (loss of postural control) (Racine, 1972).

A second baseline measurement was performed two weeks after the first. This was

done because the first baseline measurements tend to be high and thresholds drop following stimulation. The second baseline measurement was used as the baseline reference for all subsequent ADT measurements. 
After the second baseline measurement, subjects were switched from regular rat chow to the experimental diets. Control rats received the AIN-93G control diet ( $\mathrm{n}=9$ at timepoint 0 ) while experimental subjects received an AIN diet enriched in fish oil to $40 \%$ of the fat calories $(n=10$ at timepoint 0$)$. (The contents of the diets are described below) ADTs were then measured once a month for 8 months using the procedure outlined above.

\subsubsection{Diets}

The control and fish oil diets were obtained pre-mixed from Dyets Inc. (Bethlehem, PA, USA). The control diet consisted of the AIN-93G diet. The fat content of the control diet was derived from soybean oil.

The composition of the experimental fish oil diet was similar to that of the control diet, except that $40 \%$ of the soybean oil (i.e. $28 \mathrm{~g} / \mathrm{kg}$ ) was replaced by menhaden fish oil (Dyets Inc., Bethlehem, PA, USA). The fat content of menhaden fish oil is made up of approximately of $15 \%$ EPA and 10\% DHA. Subjects on the fish oil diet were consuming approximately 111 and $74 \mathrm{mg} / \mathrm{kg}$ per day of EPA and DHA respectively.

The compositions of the diets are presented in Table 3.1. Diets were analyzed by GC to confirm their content (below).

Diets were refreshed in the subjects' cages once every week, and were stored at $4{ }^{\circ} \mathrm{C}$ between weekly feedings. 


\subsubsection{Dietary Fatty Acid Analysis}

The fatty acid composition of the control and fish oil diets was determined by GC. The food pellets were first crushed. Analysis was then performed on three separate pellets of each diet. Total lipids were extracted and methylated as described in the General Methods section from approximately $1 \mathrm{~g}$ of diet. The free fatty acid methyl esters were analyzed on the Varian430 gas chromatograph system, as also described in the General Methods section.

Table 3.2 shows the fatty acid compositions of the control and fish oil diet. No EPA and DHA were detected in the control diet, whereas $3.8 \%$ and $2.4 \%$ of the fat composition of the fish oil diet was made up EPA and DHA respectively.

Table 3.1: Composition of the Control and Experimental Diets

\begin{tabular}{|l|c|c|}
\hline Ingredient $(\mathrm{g} / \mathrm{kg})$ & Control (AIN-93G) & Experimental (40\% fish oil) \\
\hline Casein & 200 & 530 \\
Cornstarch & 530 & 100 \\
Sucrose & 100 & 42 \\
Soybean oil & 70 & 28 \\
Fish oil & 0 & 50 \\
Cellulose & 50 & 10 \\
Vitamin mix & 10 & 35 \\
Mineral mix & 35 & 3 \\
L-Cysteine & 3 & 2.5 \\
Choline birtartrate & 2.5 & 0.014 \\
Tertbutyl hydroquinone & 0.014 & \\
\hline
\end{tabular}


Table 3.2: Percent Fatty Acid Composition of Diets

\begin{tabular}{|l|c|c|}
\hline Fatty acid (\%) & Control diet & Fish oil diet \\
\hline $14: 0$ & $0.95 \pm 0.05$ & $5.72 \pm 0.27$ \\
$14: 1 n-5$ & $0.05 \pm 0.05$ & $0.12 \pm 0.03$ \\
$16: 0$ & $15.63 \pm 0.40$ & $18.84 \pm 0.44$ \\
$16: 1 n-7$ & $0.33 \pm 0.03$ & $5.70 \pm 0.25$ \\
$17: 0$ & $0.23 \pm 0.07$ & $0.69 \pm 0.02$ \\
$18: 0$ & $4.31 \pm 0.11$ & $4.14 \pm 0.21$ \\
$18: 1 n-9$ & $18.60 \pm 0.35$ & $15.49 \pm 0.15$ \\
$18: 1 n-7$ & $1.24 \pm 0.05$ & $1.94 \pm 0.10$ \\
LA $(18: 2 n-6)$ & $51.18 \pm 0.45$ & $33.70 \pm 0.23$ \\
ALA $(18: 3 n-3)$ & $7.35 \pm 0.31$ & $5.04 \pm 0.09$ \\
$20: 1 n-9$ & 0 & $1.32 \pm 0.04$ \\
AA $(20: 4 n-6)$ & $0.05 \pm 0.08$ & $0.30 \pm 0.01$ \\
$22: 1 n-9$ & 0 & $0.35 \pm 0.01$ \\
EPA (20:5n-3) & 0 & $3.78 \pm 0.21$ \\
$22: 5 n-3$ & $0.51 \pm 0.01$ \\
DHA (22:6n-3) & $0.56 \pm 0.50$ \\
\hline
\end{tabular}

\subsubsection{Body Weight and Food Intake Measurements}

Body weight and food intake were measured for all subjects on a monthly basis, starting at the time of the first ADT measurement. To estimate food intake, food in the food hopper was weighed and then re-weighed again 3 days later. Food intake per day was estimated by calculating the difference in food over the 3 day interval and dividing by 3 days. 


\subsubsection{Sacrifice and Tissue Fixation}

The subjects were sacrificed after 8 months of threshold measurements by $\mathrm{CO}_{2}$ asphyxiation. After death, a current of $200 \mu \mathrm{A}$ was then passed between the electrode tips for 30 seconds in order to lesion the site of the electrode implant and thus to aid in histological analysis. The brains were then excised and stored in formalin for three weeks (minimum), to ensure complete fixation. The tissues were subsequently dehydrated by replacing the formalin with $20 \%$ sucrose solution, containing $0.1 \%$ sodium azide to prevent bacterial degradation of the brain samples. Samples were then stored at $4^{\circ} \mathrm{C}$.

\subsubsection{Histological Confirmation of Electrode Placement}

The right hemispheres from the implanted animals were used to confirm the position of the electrodes. The hemispheres were chilled in isopentane on dry ice and sectioned using a cryostat (Leica Instruments, Willowdale, Ontario, Canada) maintained at $-25^{\circ} \mathrm{C}$. The samples were allowed to equilibrate to this temperature for 30 minutes before slicing. Coronal sections were obtained at a thickness of $40 \mu \mathrm{m}$ and mounted onto gelatin-coated glass slides and stained with cresyl violet (Wolf, 1971). The position of the end of the tract was confirmed under light microscopy (Research Analysis System Model 421251; Amersham, MI). Only subjects with properly positioned electrodes were included in the subsequent data analysis (control $\mathrm{n}=7$, fish oil $\mathrm{n}=6$ ). 


\subsubsection{Statistical Analysis}

Differences in weight, food intake, change in threshold, afterdischarge duration and seizure severity were measured using two-way repeated measures ANOVA. Scores falling 2+ SDs from the mean were considered outliers and were removed from the analysis for that month only.

\subsection{Results}

Figure 3.2 presents the average weight $( \pm$ SEM) of experimental and control subjects over the 8 month period of the study. Weights in the two groups were similar at the start of the study (about 600g) and continued to be similar over the course of the study, although, both groups gained weight. After 8 months of treatment, subjects weighed $821.3 \pm 52.4 \mathrm{~g}$ and 832.5 $\pm 47.1 \mathrm{~g}$ in the control and fish oil groups respectively. A two-way repeated measures ANOVA found a significant effect of time on weight $(\mathrm{p}<0.05)$, but no significant effect of treatment and no interaction between time and treatment $(\mathrm{p}<0.05)$.

Figure 3.3 presents average food intake $( \pm$ SEM) over the 8 month period of the study. Intake in both groups was similar at the start of the study, being about 25-30g per day, and it remained similar throughout the study. Following 8 months of treatment, subjects consumed $22.5 \pm 4.1 \mathrm{~g}$ and $22.2 \pm 1.0 \mathrm{~g}$ per day of control and fish oil diet respectively. A two-way repeated measures ANOVA found no significant effect of time or treatment, and no interaction between time and treatment $(\mathrm{p}>0.05)$. 


\section{Effect of Chronic Fish Oil Administration on Weight}

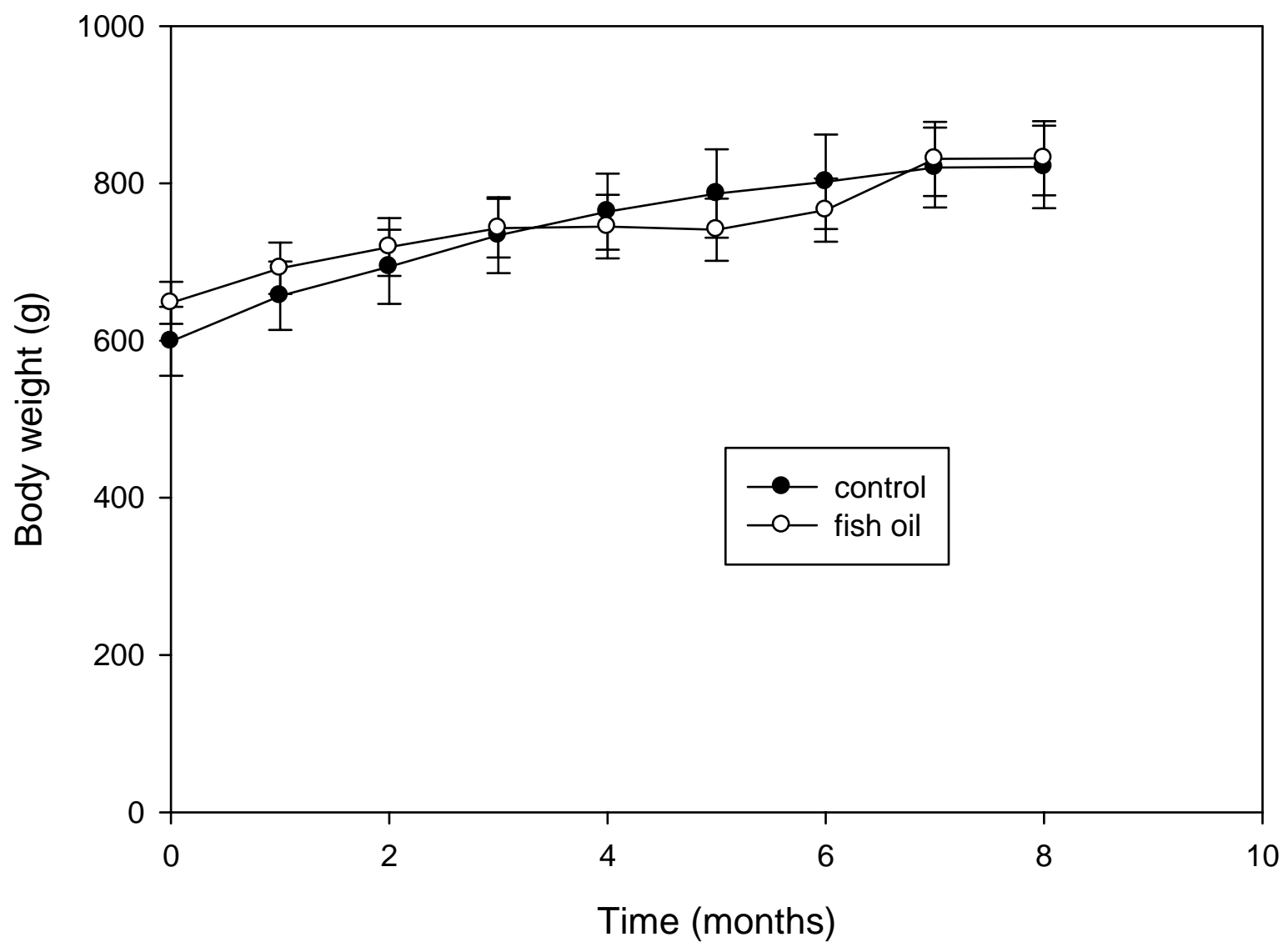

Figure 3.2. Effects of chronic dietary fish oil administration on weight. Means $(n=6-7)$ are presented \pm SEM. Control or fish oil diet was administered for a period of 8 months. Subjects' weight increased over time, regardless of the diet. A two-way repeated measures ANOVA found a significant effect of time $(\mathrm{p}<0.05)$ but not of group. 
Effect of Chronic Fish Oil Administration on Daily Food Consumption

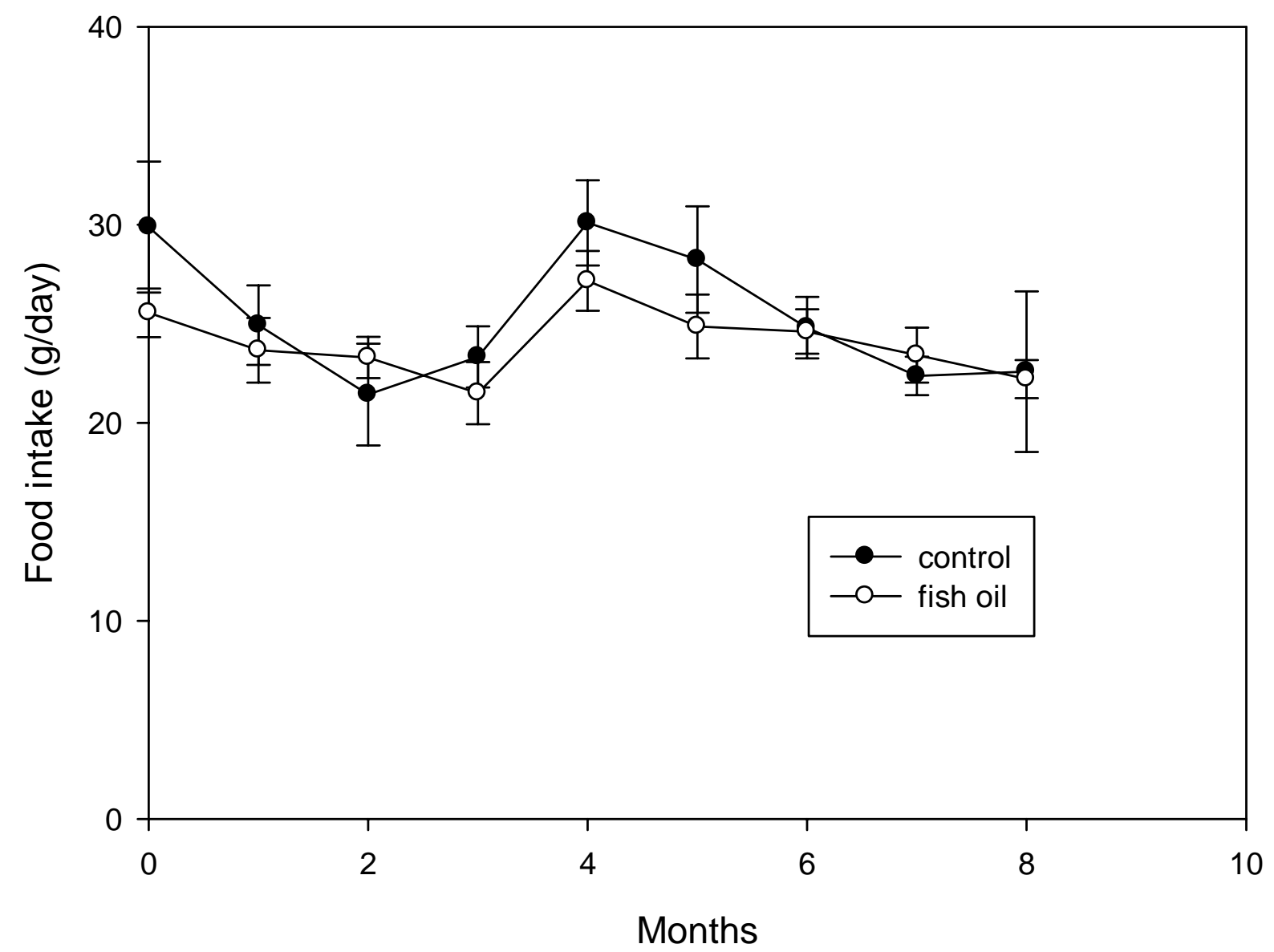

Figure 3.3. Dietary effects on food consumption. Means $(n=6-7)$ are presented \pm SEM. Diet had no effect on the subjects' food intake. Two-way repeated measures ANOVA found no significant effect of treatment or time $(\mathrm{p}>0.05)$. 
Figure 3.4 illustrates the average change in ADT as compared to baseline ADT ( \pm SEM) (One control subject whose ADT was 2+ SD from the mean of month 3 was dropped from analysis for that month). ADT dropped in subjects on the control diet whereas threshold remained stable in subjects on the fish oil diet. After 6 months of treatment, ADT had dropped by $60.0 \pm 25.2 \mu \mathrm{A}$ in the control group, whereas ADT had increased by $13.3 \pm 9.7 \mu \mathrm{A}$ in the fish oil group. Thus ADTs were similar in the two groups at the start of the experiment, but the control ADTs were much lower at the end of the experiment. A two-way repeated measures ANOVA found a significant effect of treatment and an interaction between treatment and time $(p<0.05)$. A trend towards a significant effect of time was also found ( $p=0.067)$. Tukey's post hoc tests revealed a significant difference between control and fish oil diet at months 3, 5, and $7(\mathrm{p}<0.05)$

Figure 3.5 shows the average duration of afterdischarges $( \pm$ SEM) over the 8 month period of the study (One experimental subject had a score 2+ SD from the mean at month 6 and this score was removed from the analysis for that month.). The duration of the afterdischarges increased over time in a similar fashion in both treatment groups. A two-way repeated measures ANOVA found a significant effect of time $(\mathrm{p}<0.05)$, but no effect of treatment $(p>0.05)$. Tukey's post hoc tests found a significant difference in the duration of afterdischarge between month 0 (baseline measurement prior to treatment) and month $7(\mathrm{p}<0.05)$.

Figure 3.6 displays the average behavioural seizure strength $( \pm \mathrm{SEM})$ over the 8 month period of the study. The Racine scale, which ranges from 0 to 5, was used to score the strength of the seizures. The behavioural strength of the seizures did not increase during the 8 month period of the study. Seizures in both groups averaged between 0 and 2. A two-way repeated 
Effects of Chronic Fish Oil Administration on Amygdala ADT

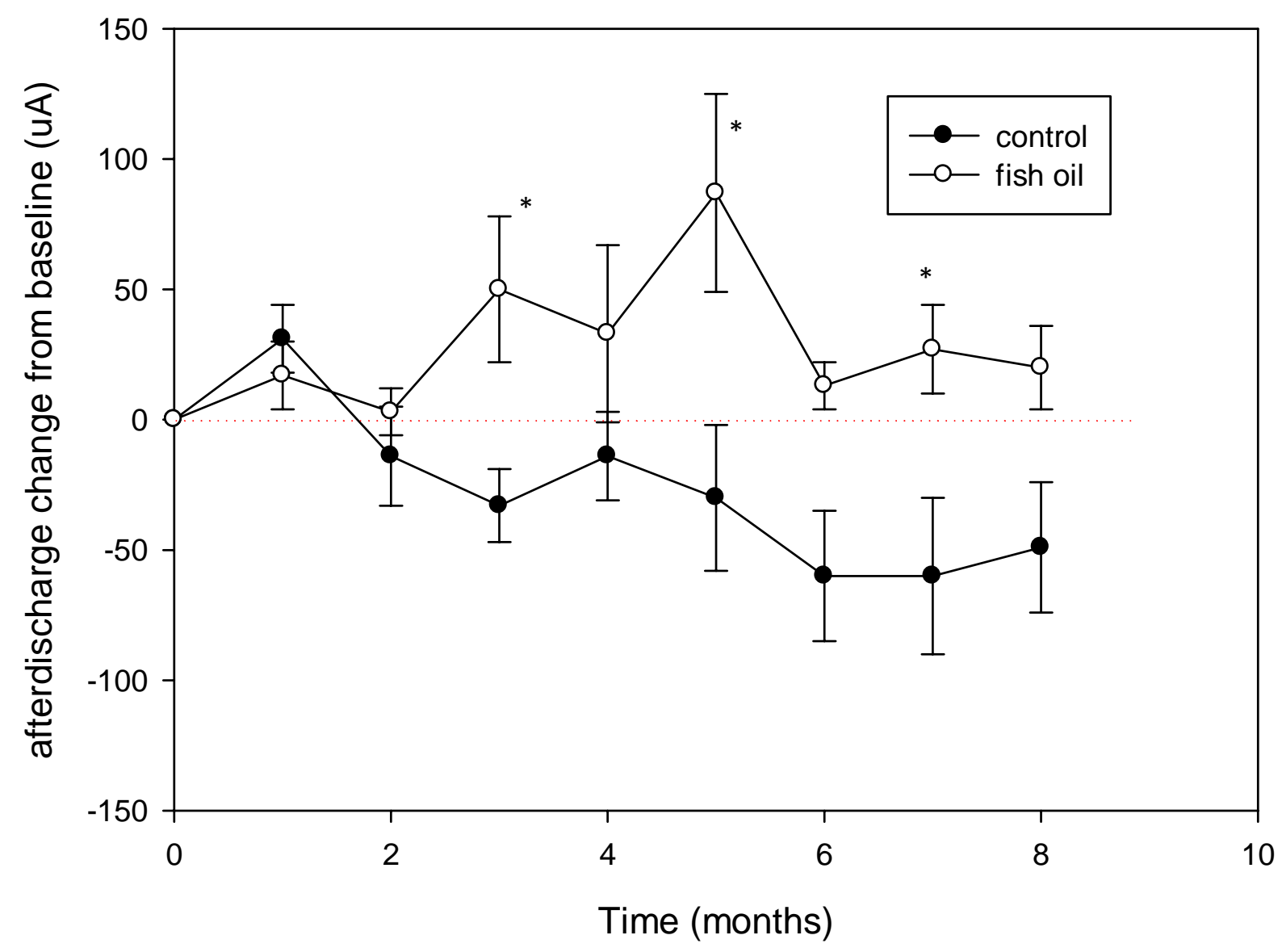

Figure 3.4. Increase in amygdala ADT compared to baseline following chronic fish oil administration. Mean changes from baseline $(n=6-7)$ are presented \pm SEM. Subjects on control diet showed a decrease in ADT, whereas subjects on fish oil diet remained approximately at baseline. A two-way repeated measures ANOVA showed a significant effect of treatment and an interaction between treatment and time

*Significant difference compared to control diet within the same month as determined by Tukey's post hoc test $(\mathrm{p}<0.05)$ 
Effects of Chronic Fish Oil Administration on Duration of Afterdischarge

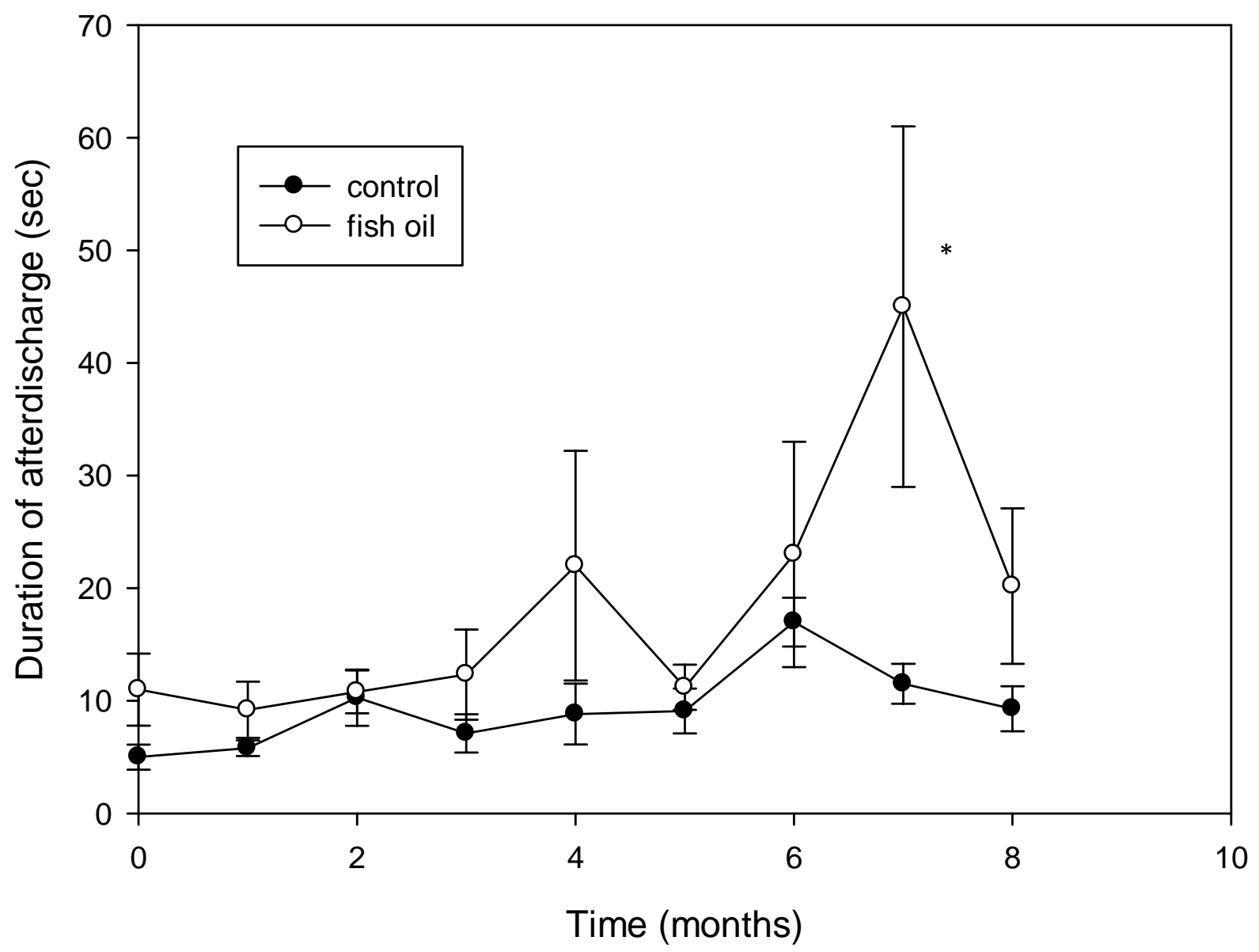

Figure 3.5. Effects of chronic fish oil administration on the duration of afterdischarge. Mean durations $(\mathrm{n}=5-7)$ are presented \pm SEM. Afterdischarge duration increased in both groups over the 8 month period of the study. A two-way repeated measures ANOVA revealed a significant effect of time on the duration of the afterdischarge $(p<0.05)$, but no effect of group membership.

*Significant difference compared to timepoint 0 as determined by Tukey's post hoc test $(\mathrm{p}<0.05)$ 
Effect of Chronic Fish Oil Administration on Behavioural Seizure Severity

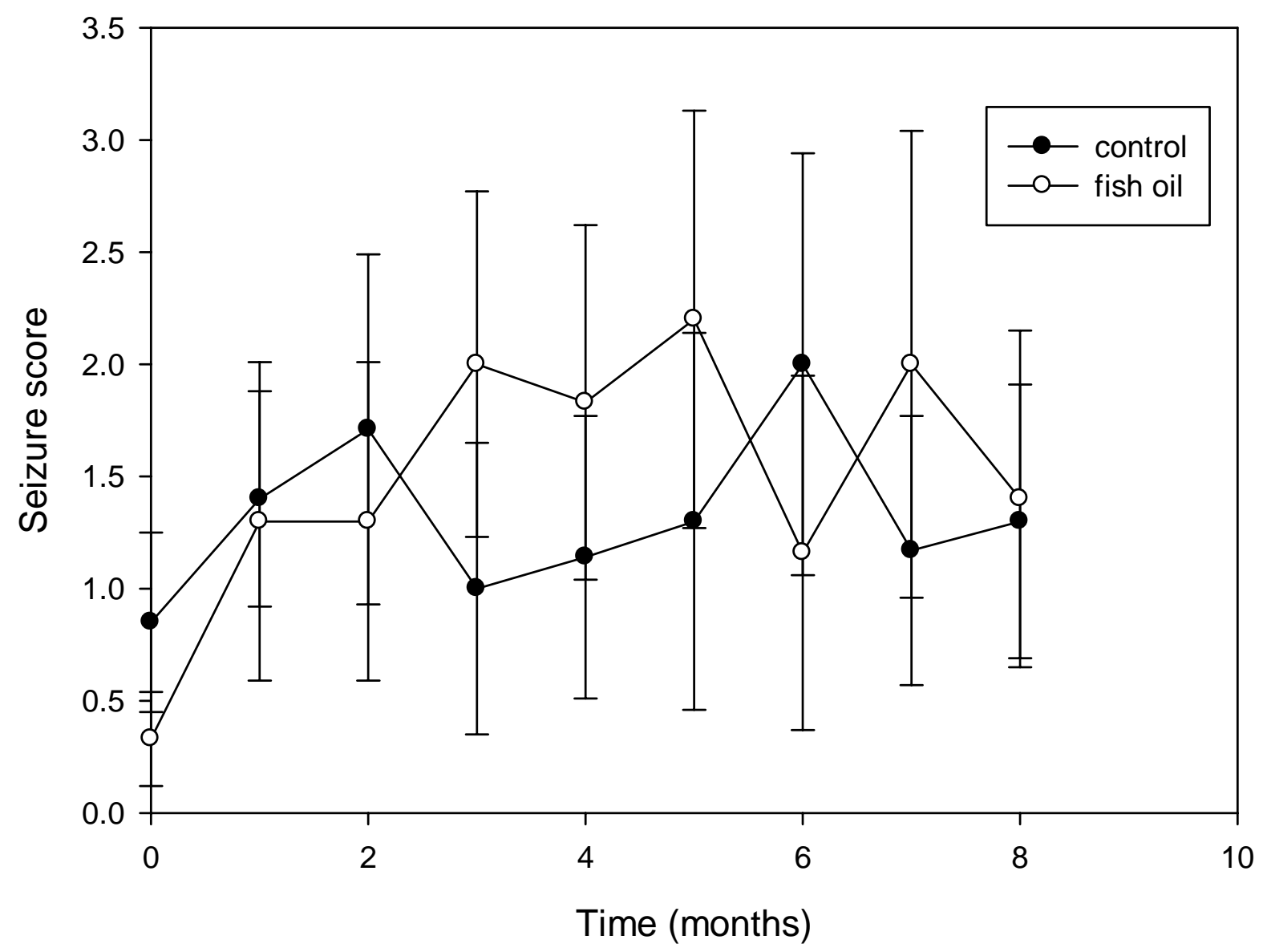

Figure 3.6. Effect of chronic fish oil on behavioural seizure severity. Seizures were scored using the Racine scale, which ranges from 0 to 5 . Mean seizure scores $(n=6-7)$ are presented \pm SEM. Seizure severity did not appear to increase during the 8 months of the study, regardless of diet treatment. A two-way repeated measures ANOVA found no effect of time or treatment $(\mathrm{p}>0.05)$. 
ANOVA found no significant effect of time or treatment on the strength of the behavioural seizures $(\mathrm{p}>0.05)$.

\subsection{Discussion}

The purpose of Experiment 3 was to confirm the anticonvulsant properties of chronic fish oil administration and to evaluate whether higher fish oil content in the diet would result in a more rapid increase in electrical ADT. In previous experiments, our group has found that chronic fish oil administration resulted in an increase in electrical ADT in the experimental group as compared to the control group. This increase, however, was only found 5 months after the start of fish oil administration, and only after the fish oil content had been increased from $20 \%$ of the total fat content to $40 \%$ of the total fat content (Taha, 2009).

In Experiment 3, it was found that chronic feeding of fish oil increased ADT in the experimental group as compared to the control group. This finding confirms the previous report of Taha et al. (2009). Likewise, as in Taha (2009), the threshold elevation was slow to develop, not being seen until after several months of feeding.

In distinction to Taha et al., however, a significant threshold elevation was seen after 3 months of feeding. This suggests that it was the rise in fish oil content which caused the threshold rise in Taha et al., and suggests that amygdala threshold will rise as quickly as cortical thresholds, provided that a sufficient amount of fish oil is used.

This finding is in agreement with a recent study by Gilby et al., which also observed that fish oil increased ADT in the amygdala following 3 months of supplementation (Gilby et 
al., 2009). It is also in general agreement with pharmacological findings that higher doses of anticonvulsant drugs are required to raise amygdala than cortical thresholds (Albright, 1983) .

The finding that it takes several months of chronic fish oil supplementation to raise seizure thresholds, now confirmed in three studies, appears to contradict previous results from our group which have shown that acute administration of n-3 PUFAs results in anticonvulsant effects within minutes (Taha, 2009). We hypothesize that difference between the two sets of results relates to the long time required to elevate unesterified DHA in serum when PUFAs are administered orally. The unesterified pool in blood is the lipid pool available for brain uptake (Hamilton et al., 2001; Ouellet et al., 2009). When injecting unesterified DHA, unesterified DHA in the blood will rise rapidly. When fish oil is given p.o., however, DHA, which comes in triglyceride form, is broken up by lipases and bile salts. Once broken up, fatty acids enter the enterocytes where they are reassembled into triglycerides in the endoplasmic reticulum and packaged into chylomicrons (CMs). The CMs then are released in the lymphatic system and then into the systemic circulation. Triglycerides are cleaved off by lipoprotein lipases, where they are incorporated into adipose tissue (Tso and Balint, 1986; Mayes and Botham, 2003; Mansbach and Siddiqi, 2010).

The half life of unsaturated fatty acids in adipose in rats has been estimated at approximately 20 days (Pihl et al., 1950). Therefore approximately only half of the fatty acids consumed on the first day would have been released from the adipose tissue after 20 days of fish oil consumption. The unesterified pool, therefore, might not increase immediately after fish oil consumption. We hypothesize that unesterified DHA in the blood will not significantly rise until after about 3 months of dietary administration - at which time thresholds should rise. Future experiments should look at unesterified blood and brain DHA concentrations in animals 
on control and experimental diets and attempt to correlate unesterified DHA changes in the experimental animals with ADT changes.

It is interesting that in the present study, the threshold rises that were seen were not accompanied by changes in electrographic or behavioural seizures. Drugs which affect ADT normally also decrease the duration of the afterdischarges and the severity of the behavioural seizures (Wlaz and Loscher, 1997; Borowicz et al., 2003). Curiously, it has been reported that phenytoin, a VDSC stabilizer like n-3 PUFAs, increases ADT and actually increases afterdischarge duration and seizure severity in some models (Ebert et al., 1997). The authors postulates that phenytoin facilitates the seizure spread to brain nuclei which generate clonic seizures (Ebert et al., 1997). It is possible that n-3 PUFAs act in a similar fashion as phenytoin. This could be a topic for future experiments. 


\section{CHAPTER 4}

Anticonvulsant Effects of Sub-Chronic Administration of DHA and DHA EE in the Maximal PTZ Seizure Model 


\subsection{Rationale}

Experiment 4 was designed to explore the anticonvulsant effects of DHA and DHA EE when administered sub-chronically by i.p. injection for a period of 2 weeks. It was an attempt to reproduce the results of Bruzzese (2005)

In our laboratory, work on the n-3 PUFAs had begun with an attempt to replicate the studies of Yehuda who had claimed that the sub-chronic (three weeks) i.p. administration of short-chain n-3 PUFAs (40 mg/kg of the SR-3 mixture) produced dramatic anticonvulsant effects in 4 different seizure models (Yehuda et al., 1994; Rabinovitz et al., 2004). Our group was unable to replicate these dramatic results, although much more modest effects were seen at $200 \mathrm{mg} / \mathrm{kg}$. (Taha et al., 2006). At $200 \mathrm{mg} / \mathrm{kg}$ we saw an increase in latency onset, but none of the seizure suppression previously reported by Yehuda et al. (Yehuda et al., 1994; Rabinovitz et al., 2004; Taha et al., 2009b).

More recently, we discovered that a group in 2005 had published a patent claiming to replicate Yehuda's dramatic results in an experiment that had replaced the SR-3 mixture with sub-chronic (14 days) DHA EE (Bruzzese, 2005). The present experiment was designed to determine whether these dramatic results could be replicated, and also whether the free form of DHA would have the same effect.

\subsection{Methods}

Methods were generally designed to match those of Bruzzese (2005). Certain differences are noted in the Discussion section below. 


\subsubsection{Subjects}

Male 53 day-old Wistar rats were obtained from Charles River (La Prairie, Qc) and housed as described in the General Methods section. Body weight was measured daily for all subjects during the period of injections.

\subsubsection{Drugs}

DHA and DHA EE were obtained as described in the General Methods section. Solutions were prepared by mixing $140 \mu \mathrm{l}$ of DHA or DHA EE with $90 \mathrm{mg}$ of BSA per ml of saline. The final concentration of the stock solution was $128.8 \mathrm{mg} / \mathrm{ml}$ and $127.4 \mathrm{mg} / \mathrm{ml}$ for DHA and DHA EE respectively. A vehicle solution containing 90mg of BSA dissolved in $1 \mathrm{ml}$ of physiological saline was given to control subjects.

\subsubsection{Drug Administration}

Two groups of 10 animals received a dose of $50 \mathrm{mg} / \mathrm{kg}$ i.p. of either DHA or DHA EE for 14 consecutive days. Vehicle solution, volume matched to the DHA group, was administered to a third group of control animals. 


\subsubsection{Seizure Testing}

On day 15 , each animal received $105 \mathrm{mg} / \mathrm{kg}$ i.p. of PTZ (determined to be optimal PTZ dose in a pilot study, $n=10$ ). Latency to tonic forelimb extension was measured, as it had been in the Bruzzese study.

\subsubsection{Statistical Analysis}

Differences in body weights were analyzed using a two-way repeated measures ANOVA. Differences in seizure latencies were not normally distributed and were therefore analyzed using a non-parametric analysis of variance on ranks (Kruskal-Wallis test).

\subsection{Results}

Figure 4.1 presents mean $( \pm \mathrm{SD})$ daily body weights for the different groups of subjects during the period of injections. As indicated, body weight increased over time in all treatment groups. A two-way ANOVA revealed a significant effect of time and a significant interaction between time and treatment $(\mathrm{p}<0.05)$. There was no significant effect of treatment per se. Tukey's post hoc test found no difference in weight between either experimental treatment group and the control treatment group $(\mathrm{p}<0.05)$.

Figure 4.2 shows the average latencies $( \pm \mathrm{SD})$ to tonic-clonic seizures of all treatment groups in the maximal PTZ model. (Four animals were removed from analysis - Saline $\mathrm{n}=2$, 
Effect of Chronic Administration of DHA and DHA EE on Weight of Male Wistar Rats

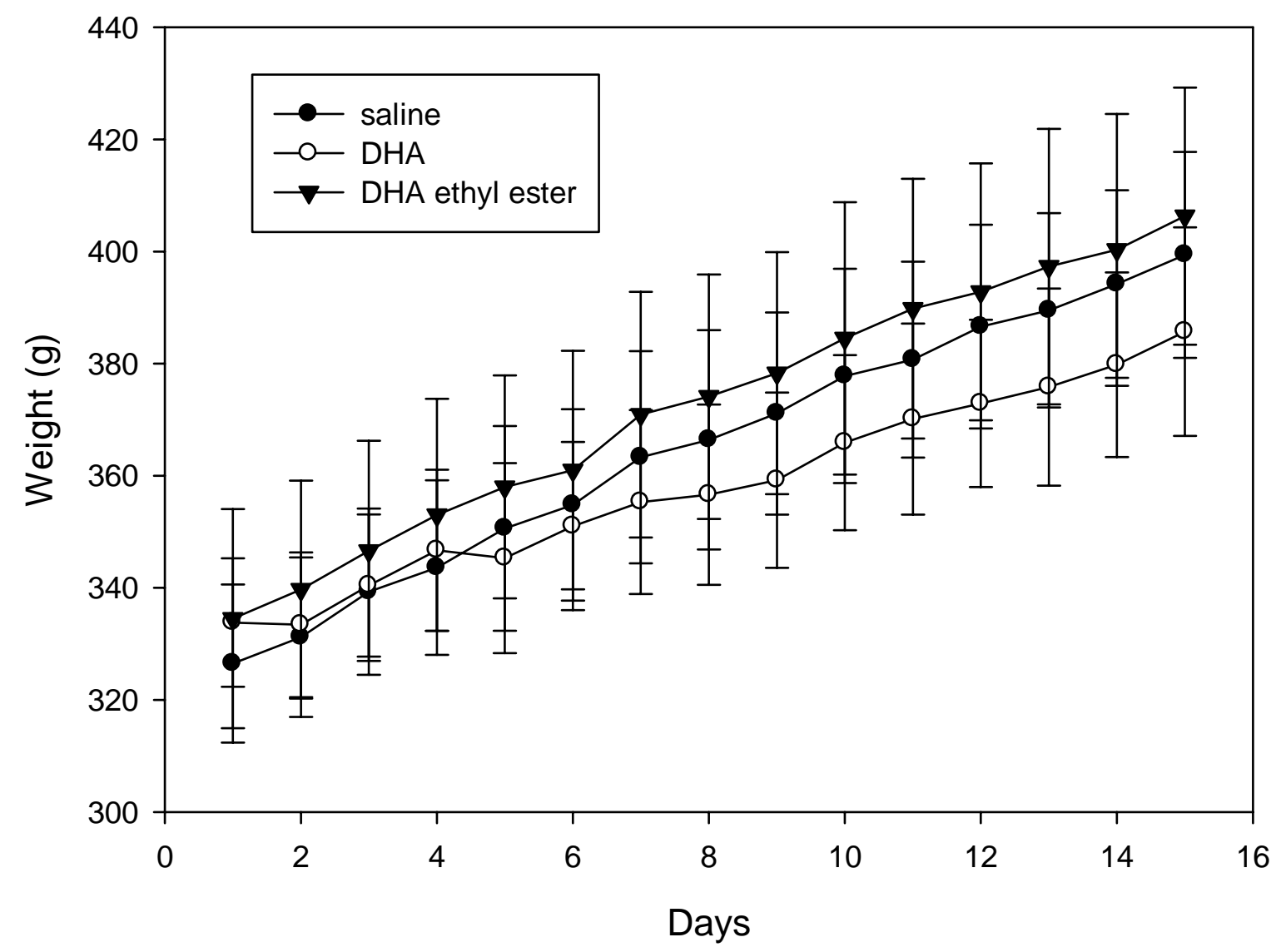

Figure 4.1. Effect of chronic administration of either DHA or DHA EE on weight. Means $(\mathrm{n}=10)$ are presented $\pm \mathrm{SD}$. Weights of rats receiving either DHA or DHA EE did not significantly differ from the saline control group as indicated by two-way repeated measures ANOVA $(p>0.05)$. 
Anticonvulsant Effect of 14 Days Chronic Administration of DHA and DHA EE in the Maximal PTZ seizure Model

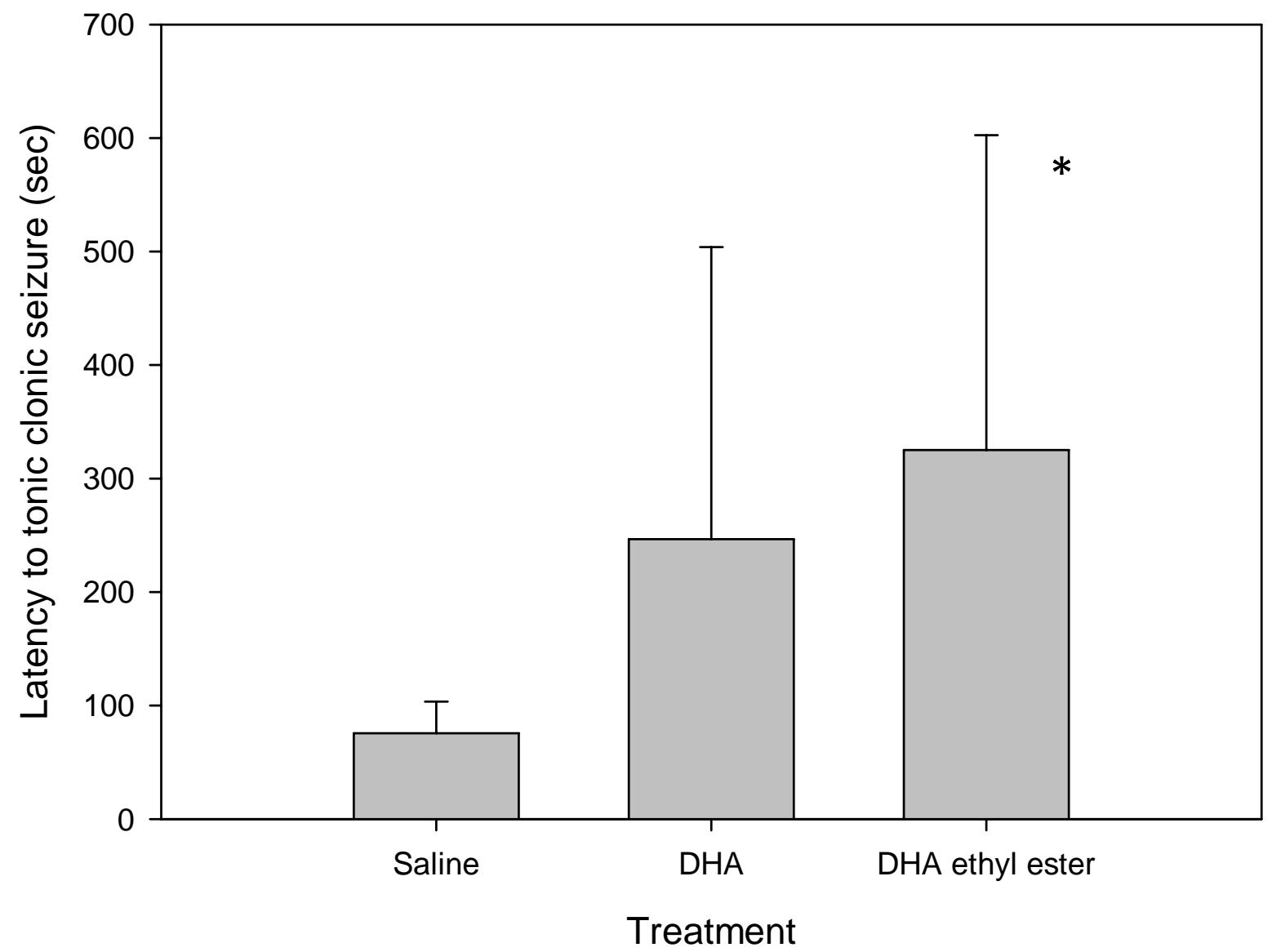

Figure 4.2. Elevation in latency to maximal PTZ seizures following the chronic administration of DHA or DHA ethyl ester for a period of 14 days. DHA and DHA ethyl ester were administered at $50 \mathrm{mg} / \mathrm{kg}$ while PTZ was administered at $105 \mathrm{mg} / \mathrm{kg}$. Means $(\mathrm{n}=8-9)$ are presented $\pm \mathrm{SD}$

*Significant difference as compared to the saline group as determined by Dunn's post hoc test $(\mathrm{p}<0.05)$ 
DHA $n=1$, DHA EE $n=1-$ due to the fact that they did not seize). As illustrated by Figure 4.2, both DHA and DHA ethyl appeared to have anticonvulsant properties, since they had longer latencies than the control group. They did not appear to differ between themselves. The nonparametric Kruskal-Wallis test revealed a significant difference among the 3 groups $(\mathrm{p}=0.041)$. The latency for the DHA EE group was significantly higher than the saline control group (Dunn's post hoc test, $\mathrm{p}<0.05$ ), but the DHA group was not. As the 2 DHA groups are not significantly different from each other (Mann-Whitney $U$ test, $p>0.05$ ), they were combined for statistical purposes. When combined, they were significantly different from the control group, as indicated by the Mann-Whitney $U$ test $(\mathrm{p}=0.013)$.

\subsection{Discussion}

Experiment 4 was designed to test the anticonvulsant properties of DHA and its EE form when given chronically over a 2 week period in the maximal PTZ model. It was found that both DHA and DHA EE increased latency to seizure onset in the PTZ test, and - when the groups were combined - this effect was statistically significant.

Our results differed from those previously reported by Bruzzese (Bruzzese, 2005) in that we did not observe seizure suppression - although we did get an increase in seizure latency. We also saw a good deal more variability in the data than had been evident in Bruzzese's data.

Some differences existed between the two experiments which must be considered. Our group used Wistar rats - to stay consistent with the remainder of our experiments - whereas 
Bruzzese had used Sprague Dawley rats. This may have had an effect on the results, although it is hard to imagine that strain could have such a large effect.

Bruzzese also made use of tocopherol in his experiment, a vitamin E compound which would prevent the oxidation of fatty acids. We did not use tocopherol in our experiment. Our stock solution was stored at -80 , however, to prevent oxidation between injections. Moreover, our group had previously used tocopherol in our attempts to replicate Yehuda et al., but had failed to obtain seizure suppression (Yehuda et al., 1994; Taha et al., 2006; Taha et al., 2009a). We are, therefore, not convinced that the use of tocopherol explains the differences between our results and the results obtained by Bruzzese and his group.

One curiosity in Bruzzese's data is that the latencies to seizure onset were extremely short - far shorter than any we have ever seen in our experiments. Future experiments might involve a strict replication of Bruzzese's study. 


\section{CHAPTER 5}

Replication of the Inverted U Dose Response Curve for Acutely Administered DHA in the Maximal PTZ Seizure Model with Measures of Blood Levels of DHA 


\subsection{Rationale}

The purpose of Experiments 5A and B was to confirm the existence of an inverted Udose-response curve when DHA was administered s.c., and - after it was confirmed - to determine the serum DHA levels associated with medium (effective) and high (ineffective) doses of DHA.

In a previous pilot study in our laboratory $(n=3)$, Taha had found that the s.c. administration of DHA increased latencies to maximal DHA seizures 1 hour after injection, but he had also found that the effects were lost with higher doses of DHA (Taha et al., 2010).

The first experiment in this chapter (Experiment 5A) was designed to confirm this inverted-U dose-response relationship, since true inverted dose-response curves are rare in pharmacology. Larger N's of 7-10 per dose group were used. Based on the results of the pilot study, we hypothesized that an inverted-U shaped dose-response relationship would be found.

In a second experiment (Experiment 5B), we measured serum DHA levels following medium (effective) and high (ineffective) s.c. DHA injections. Our hypothesis was that an elevation in free serum DHA would occur at the DHA dose that caused anticonvulsant effects, but that higher doses would produce lower serum levels - perhaps because DHA might be trapped at the injection site following higher doses. 


\subsection{Methods}

\subsubsection{Subjects}

Male 53 days old Wistar rats (Charles River Canada) served as subjects. Subjects were singly housed, with water and food available ad libitum as described as in the General Methods section.

\subsubsection{Drugs and Seizure Testing - Experiment 5A}

DHA and OA solution were prepared by mixing $140 \mu$ l of DHA or OA with $90 \mathrm{mg}$ of BSA per $\mathrm{ml}$ of saline. The final concentration of the stock solution was $128.8 \mathrm{mg} / \mathrm{ml}$ and $124.6 \mathrm{mg} / \mathrm{ml}$ for DHA and OA respectively. A vehicle solution containing $90 \mathrm{mg}$ of BSA dissolved in $1 \mathrm{ml}$ of physiological saline was administered to the control group.

At the time of seizure testing, experimental animals received one of the following doses of DHA: 100, 200, 400, or $600 \mathrm{mg} / \mathrm{kg}$ s.c. Control animals received $400 \mathrm{mg} / \mathrm{kg}$ of OA s.c., a dose matched to the expected effective dose of DHA. One hour later, $105 \mathrm{mg} / \mathrm{kg}$ (determined by pilot data, $\mathrm{n}=10$ ) of PTZ was administered i.p. as described in the General Methods. Latency to tonic-clonic seizures was measured.

\section{Drugs and Blood Collection - Experiment 5B}

In our second experiment, each animal received one of the following treatments: $400 \mathrm{mg} / \mathrm{kg} \mathrm{OA}$, or $400 \mathrm{mg} / \mathrm{kg}$ DHA or $600 \mathrm{mg} / \mathrm{kg}$ DHA s.c. These doses were chosen because 
the $400 \mathrm{mg} / \mathrm{kg}$ dose appeared to be anticonvulsant in Experiment 5A, but the effects were lost at the higher dose of $600 \mathrm{mg} / \mathrm{kg}$. The OA injection was a control, matched to the effective dose of DHA.

One hour following the s.c injections, the subjects were anesthetized with $50 \mathrm{mg} / \mathrm{kg}$ of ketamine (Bimedia-MCT, Cambridge, ON) and $10 \mathrm{mg} / \mathrm{kg}$ of xylazine (Bayer, Leverkusen, Germany), and subsequently decapitated. Truncal blood was collected and placed on ice. Blood was spun for $20 \mathrm{~min}$ at $5000 \mathrm{RPM}$ and serum was collected and stored at $-80^{\circ} \mathrm{C}$ until analysis.

\subsubsection{Fatty Acid Extraction and Analysis}

Fatty acid analysis was performed as outlined in the General Methods.

\subsubsection{Statistical Analysis}

Differences in latencies to tonic-clonic seizures and DHA concentrations within each of the fatty acid pools were analyzed using one-way ANOVAs. Tukey's post-hoc tests were applied when appropriate.

\subsection{Results}

Figure 5.1 presents the average latencies to tonic-clonic seizures of the different doses 1 hour after s.c. injection. Animal falling 2 standard deviation outside of the average were considered outliers and removed from analysis $(200 \mathrm{mg} / \mathrm{kg} \mathrm{n}=1,400 \mathrm{mg} / \mathrm{kg} \mathrm{n}=1,600 \mathrm{mg} / \mathrm{kg} \mathrm{n}=1$, 
OA $n=1)$. Figure 5.1 illustrates a rise in latency to tonic-clonic seizures with increasing doses. The latency returned to baseline levels, however, at the highest dose. A one-way ANOVA was significant, and post-hoc tests revealed significant differences between the $400 \mathrm{mg} / \mathrm{kg}$ DHA group and both the OA group and the $600 \mathrm{mg} / \mathrm{kg}$ group $(\mathrm{p}<0.05$, Tukey's post hoc test).

The data related to unesterified DHA concentrations 1 hour after s.c. injection are presented in Figure 5.2. As indicated, unesterified serum DHA concentrations were lowest after treatment with OA (control), higher after treatment with $400 \mathrm{mg} / \mathrm{kg}$ of DHA and highest after injection of $600 \mathrm{mg} / \mathrm{kg}$ DHA. A one-way ANOVA indicated that there were significant differences among this group of means $(\mathrm{P}<0.05)$. Tukey's post-hoc tests indicated that the levels of unesterified DHA were significantly elevated after both the $400 \mathrm{mg} / \mathrm{kg}$ and the 600 $\mathrm{mg} / \mathrm{kg}$ injections, as compared to the levels in OA-injected subjects. Levels after the 400 and $600 \mathrm{mg} / \mathrm{kg}$ doses did not differ significantly from each other $(\mathrm{P}>0.05)$.

Figure 5.3 presents mean values for serum phospholipids, cholesteryl esters, and triglycerides 1 hour after s.c. injections of OA or DHA. Values were similar in all groups. A one-way ANOVA revealed no significant differences among the means in any of the three esterified lipid pools. 
s.c. DHA Dose-Response Curve in the Maximal PTZ Model

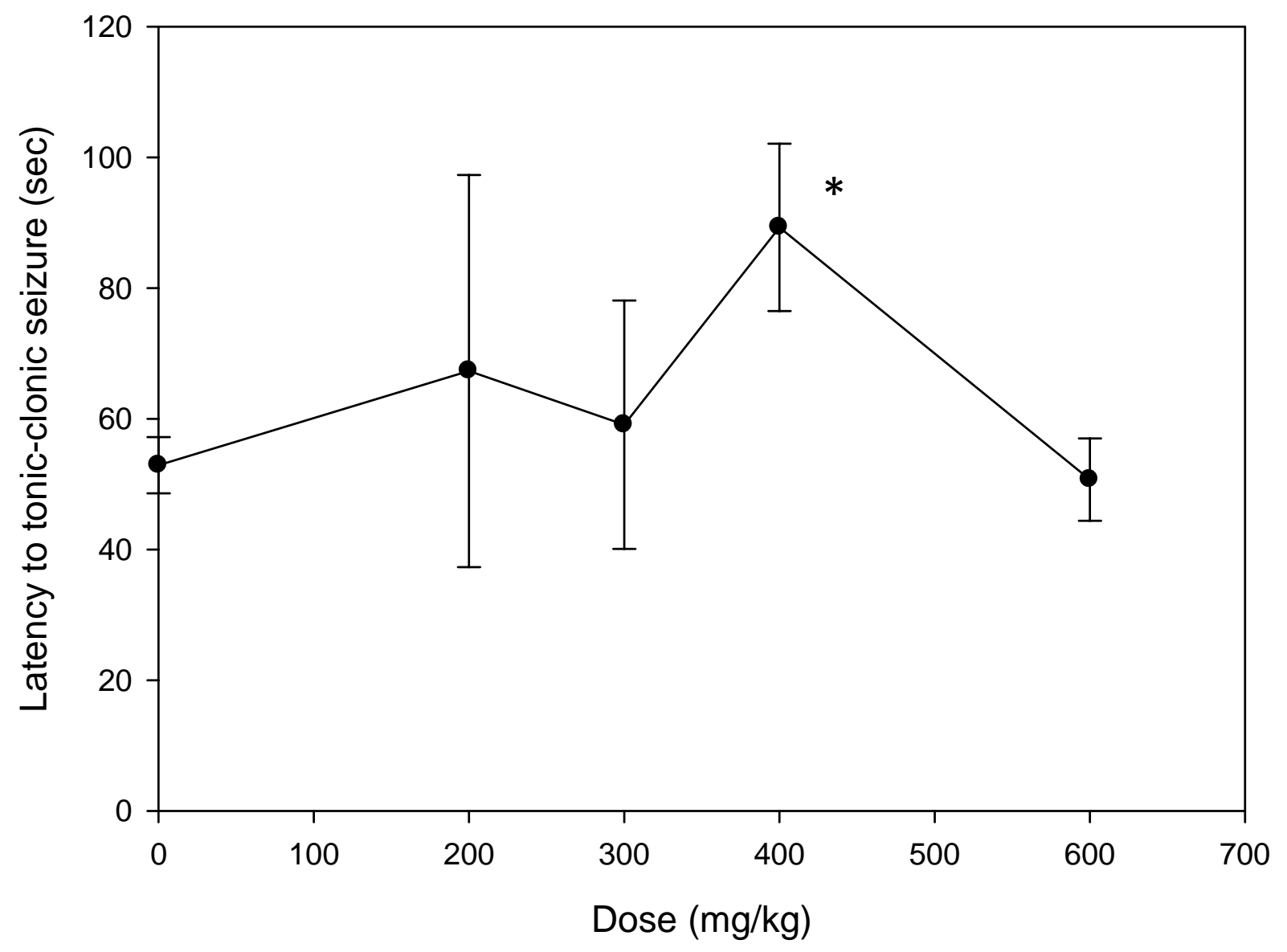

Figure 5.1. Latency to onset of tonic-clonic seizures $1 \mathrm{hr}$ after s.c. of different doses of DHA or $400 \mathrm{mg} / \mathrm{kg}$ of OA $(0 \mathrm{mg} / \mathrm{kg})$. Data are presented in mean $\pm \mathrm{SD}(\mathrm{n}=7-10$ per group $)$.

* Indicates $\mathrm{p}<0.05$ as compared to OA and $600 \mathrm{mg} / \mathrm{kg}$ (one-way ANOVA followed by Tukey's post-hoc tests) 
Unesterified DHA Concentrations in Serum Following s.c. OA or DHA Injections

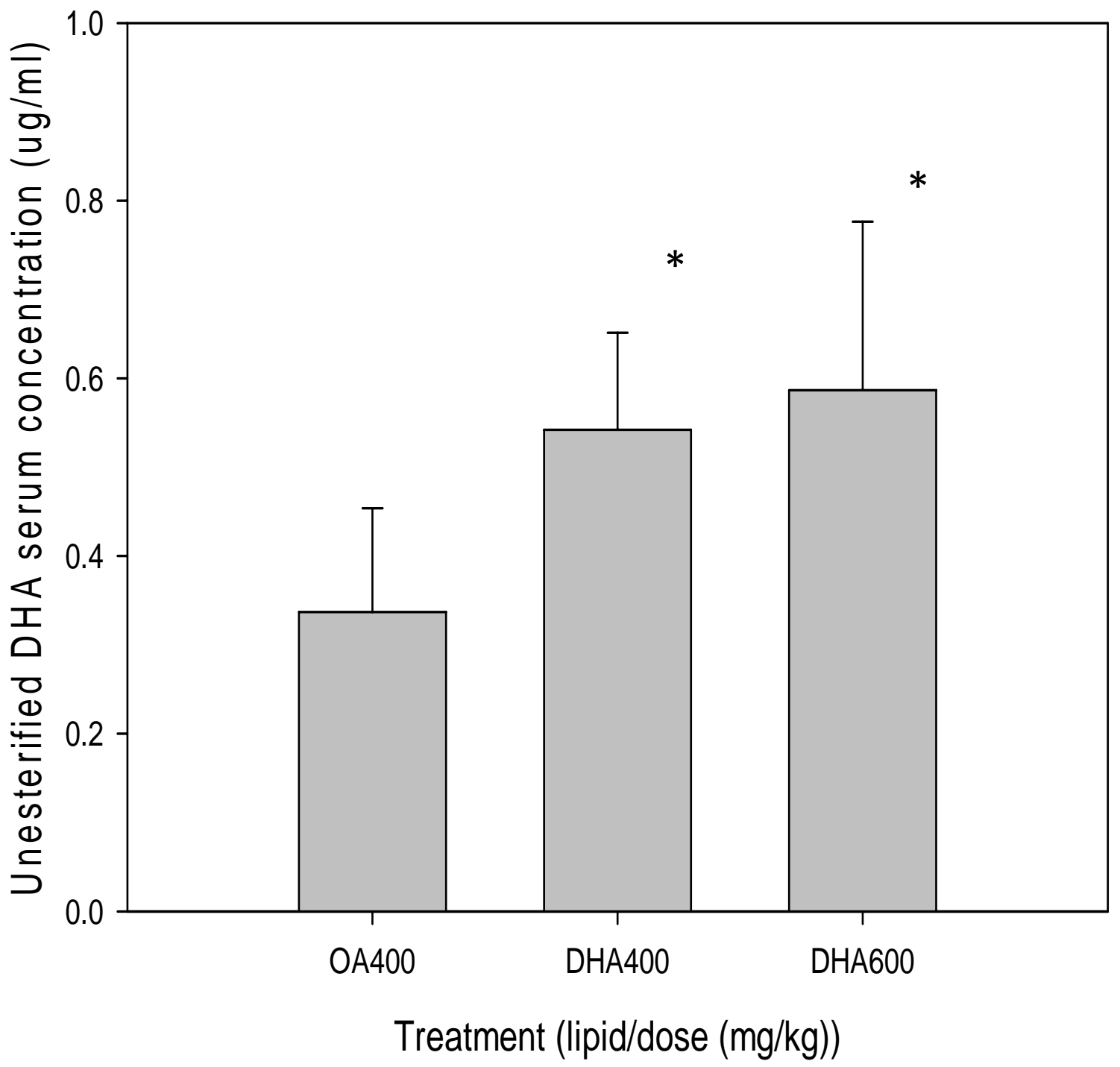

Figure 5.2. Unesterified DHA serum concentrations $(\mu \mathrm{g} / \mathrm{ml}) 1 \mathrm{hr}$ after s.c injections of OA or DHA. Data are presented as means $\pm \mathrm{SD}(\mathrm{n}=8-10$ per group).

* indicates $\mathrm{P}<0.05$ as compared to OA $400 \mathrm{mg} / \mathrm{kg}$ (one-way ANOVA followed by Tukey's post-hoc tests). No difference was found between DHA $400 \mathrm{mg} / \mathrm{kg}$ and DHA $600 \mathrm{mg} / \mathrm{kg}$ $(\mathrm{P}>0.05)$ 
Esterified DHA Concentrations in Serum Following s.c. OA or DHA Injections

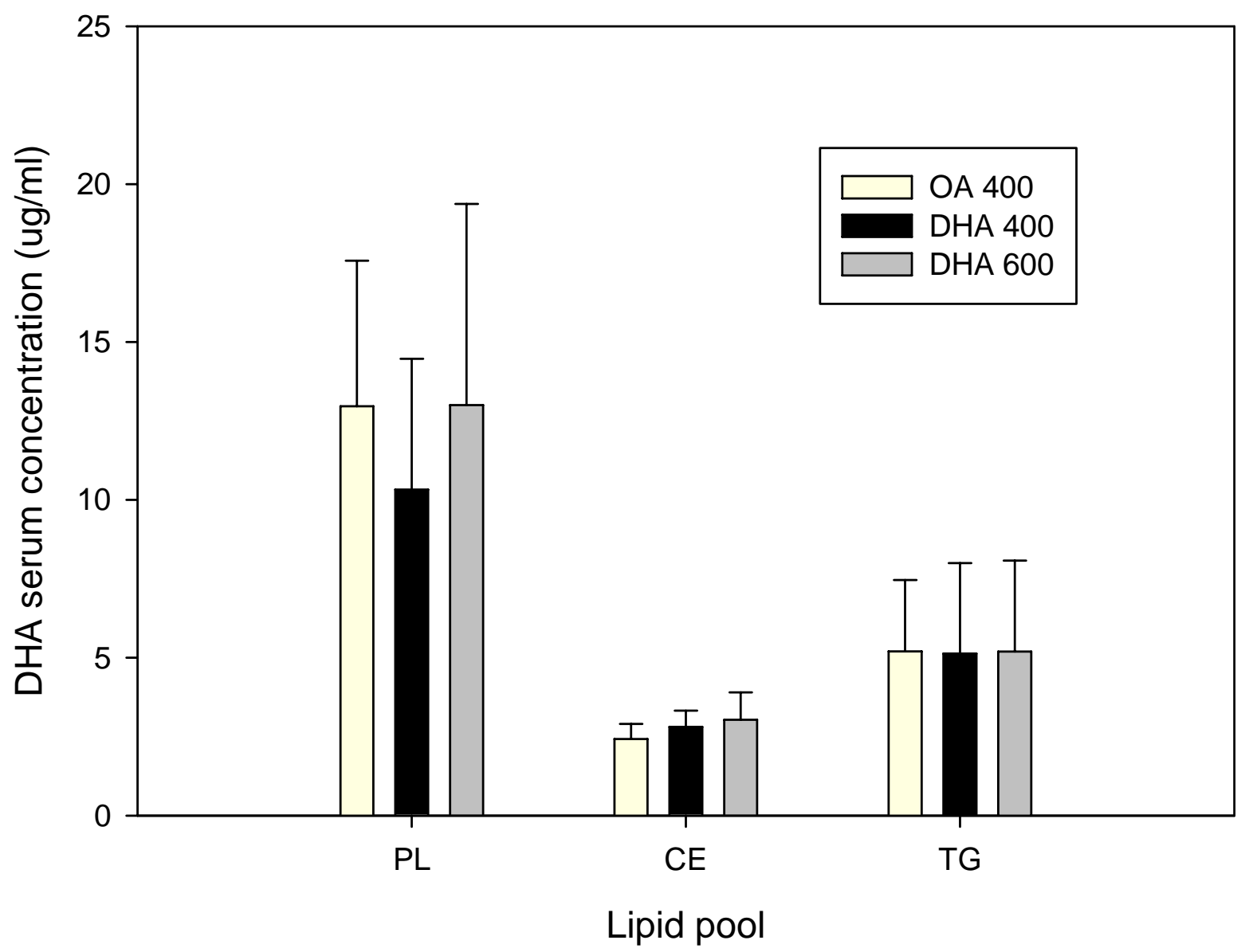

Figure 5.3. Concentrations of unesterified DHA in serum $(\mu \mathrm{g} / \mathrm{ml})$ in various lipid pools $1 \mathrm{hr}$ following s.c. injection of OA or DHA. Data are presented as means \pm SD $(n=8-10$ per group). Lipid pools: $\mathrm{PL}=$ phospholipid, $\mathrm{CE}=$ cholesteryl ester, $\mathrm{TG}=$ triglyceride One-way ANOVA found no significant effects of treatment. 


\subsection{Discussion}

The present experiments were designed to confirm the inverted $U$ dose-response relationship previously seen when DHA was administered s.c., and to measure the serum DHA levels after medium (effective) and high (ineffective) doses.

As expected, in Experiment 5A the administration of DHA s.c. produced an anticonvulsant effect at the $400 \mathrm{mg} / \mathrm{kg}$ dose. These effects were lost, however, at the higher dose of $600 \mathrm{mg} / \mathrm{kg}$. Thus, the present study confirmed both that the s.c. administration of DHA results in an elevation of seizure threshold, and also that the effect on threshold is lost at higher DHA doses, producing an "inverted-U” dose-response curve, as we hypothesized.

In Experiment 5B, the administration of DHA s.c. resulted in an elevation of serum unesterified DHA 1 hour following administration. The $400 \mathrm{mg} / \mathrm{kg}$ dose increased unesterified DHA serum level, which was also the first dose to produce significant anticonvulsant effects. Thus, at $400 \mathrm{mg} / \mathrm{kg}$, at least, our first hypothesis was confirmed in that the elevation in seizure threshold correlated with an elevation in unesterified DHA in the serum. This also appeared to be in agreement with the fact that it is the FFA pool in serum that is available to the brain (Hamilton et al., 2001; Ouellet et al., 2009). At this time, no change was observed in the esterified pool.

Our second hypothesis was that that DHA lost its potency at higher doses because it came out of solution at the point of injection. A similar suggestion has been made for phenytoin when administered intramuscularly and intraperitoneally (Serrano and Wilder, 1974; McNamara et al., 1989). The data from Experiment 5B, however, did not appear to support this hypothesis. The serum levels of unesterified DHA found after the $600 \mathrm{mg} / \mathrm{kg}$ injection were 
just as high as the serum levels after the $400 \mathrm{mg} / \mathrm{kg}$. No large reduction in serum DHA occurred at $600 \mathrm{mg} / \mathrm{kg}$ to explain the loss of DHA's effect on seizure threshold.

It is interesting to note, however, that the $600 \mathrm{mg} / \mathrm{kg}$ injection dose $(50 \%$ higher than $400 \mathrm{mg} / \mathrm{kg}$ ) produced unesterified serum DHA levels that were only slightly (non-significantly) higher than the $400 \mathrm{mg} / \mathrm{kg}$ injection It is possible that micelles may have formed at the site of injection and entered the bloodstream. DHA trapped in micelles might not be available to the brain. It must be noted that our assays would not have distinguished between unesterified DHA bound to albumin in the serum and unesterified DHA that had come out of solution at the site of injection in the form micelles. A formation of micelles at the site of injection could explain why the $600 \mathrm{mg} / \mathrm{kg}$ dose did not result in an increase in serum concentration compared to the $400 \mathrm{mg} / \mathrm{kg}$ dose.

The current hypothesis on brain lipid uptake is that only unesterified lipids bound to albumin are available for brain uptake (Hamilton et al., 2001; Chen et al., 2008b; Ouellet et al., 2009). Perhaps DHA in micelle form may not available for brain uptake. Thus, even though blood concentrations between the $400 \mathrm{mg} / \mathrm{kg}$ and $600 \mathrm{mg} / \mathrm{kg}$ doses were similar, it is possible that brain concentrations may have been different due to micelle formation, explaining the difference in effects. This could be tested in future experiments.

Future studies should therefore involve measurements of radiolabelled unesterified DHA in the serum and brain after effective $(400 \mathrm{mg} / \mathrm{kg})$ and ineffective $(600 \mathrm{mg} / \mathrm{kg})$ injections to elucidate whether or not different amounts of DHA are taken up by the brain between the two concentrations. 
CHAPTER 6:

Dose- and Time-Concentration Curves Following i.v. DHA Infusion 


\subsection{Rationale}

As indicated in Chapter 5, experiments with s.c. injections of DHA produced data inconsistent with what is known about the pharmacokinetics of DHA. We hypothesized that DHA might be trapped and/or coming out solution at the site of injection when injected s.c., and that the injection site might therefore be acting as a "slow release" locus. It was therefore decided to switch to i.v. administration to see whether the pharmacokintetics of DHA would be simpler when it was administered i.v.

Our first studies involved measurements of serum concentrations rather than of seizure suppression, since concentration seemed to be a simpler parameter to work with. Before seizure testing, we wanted to see with i.v. infusions: 1) whether stable levels of DHA could be achieved in the serum, 2) how long they would take to appear, 3) how high they would be, and 4) how long they would last after the infusion had been stopped.

Infusions were done, rather than bolus injections, since early pilot experiments showed that bolus injections could stop the heart. The i.v. injection of a bolus $400 \mathrm{mg} / \mathrm{kg}$ dose, for instance, resulted in 50\% mortality in the group of pilot rats tested. The mortality was most likely due to bradychardia, a common side effect of bolus injection of sodium channel blockers (Jenkins and Spector, 1973). Doses were therefore administered as i.v. infusions over the course of 5 minutes.

The purpose of the first experiment in this chapter (Experiment $6 \mathrm{~A}$ ), therefore, was to evaluate serum concentrations of DHA following the infusion of DHA directly into the systemic circulation via the tail vein. Concentrations related to different doses of DHA and different times after injection were explored. The purpose of the second experiment 
(Experiment 6 B) was to evaluate how long DHA remained within the systemic circulation following the discontinuation of DHA infusion.

\subsection{Methods}

\subsubsection{Subjects}

Male 350g Wistar rats were obtained from Charles River (La Prairie, Qc). The change in weight was necessitated by the fact that larger animals were needed to facilitate angicath (cannula) placement. Animals were singly housed, with water and food available ad libitum, as described in the General Methods section.

\subsubsection{Drug Solutions}

Solutions were prepared by mixing DHA and BSA, in a ratio of $1.43 \mathrm{mg}$ per of DHA to mg of BSA, in physiological saline. The ratio was maintained for all doses by diluting the original stock solution by serial dilution. The concentrations of the stock solutions were 151.8, $75.9,38.0,19.0$, and $9.5 \mathrm{mg} / \mathrm{ml}$ for the $400 \mathrm{mg} / \mathrm{kg}, 200 \mathrm{mg} / \mathrm{kg}, 100 \mathrm{mg} / \mathrm{kg}, 50 \mathrm{mg} / \mathrm{kg}$, and $25 \mathrm{mg} / \mathrm{kg}$ doses respectively. Different concentrations were used in order to maintain constant infusion volume of approximately $1 \mathrm{ml}$ of solution. 


\subsubsection{Procedure for Drug Infusion}

Subjects were placed under a heat lamp for 20 minutes to cause vasodilation. A 24 gauge angiocath was then inserted in both tail veins. The infusion line was connected to one angiocath (left). The second angiocath (right) was used to withdraw blood samples.

For the dose and time-concentration study (Experiment 6A), subjects received 25, 50, 100,200 , or $400 \mathrm{mg} / \mathrm{kg}$ of DHA, infused over 5 minutes, in a volume of approximately $1 \mathrm{ml}$ of solution. (Volume of injection varied slightly depending on weight.) Blood was sampled before and at different times during the infusion (below).

For the time after discontinuation-concentration study (Experiment 6B), subjects were initially infused with either 25 or $100 \mathrm{mg} / \mathrm{kg}$ of DHA infused over 5 minutes, in a volume of approximately $1 \mathrm{ml}$ of solution. Different subjects from the subjects in Experiment $6 \mathrm{~A}$ were

used due to the large volume of blood needed to be collected for the assay (The Division of Comparative Medicine limits the amount of blood that can be extracted from a single subject). The $100 \mathrm{mg} / \mathrm{kg}$ DHA dose was chosen because it was the highest dose that did not generate major side effects in Experiment 6A (Behavioural abnormalities were observed in some subjects at higher doses). The lower dose, $25 \mathrm{mg} / \mathrm{kg}$, was used to determine whether kinetics would vary when the dose was changed. After 5 minutes, the infusion was stopped. Blood was sampled at the start and end of the 5-minute infusion and at varying times after the infusion was stopped. 


\subsubsection{Procedures for Blood Collection}

In the dose-concentration study (Experiment $6 \mathrm{~A}$ ), approximately $0.2 \mathrm{ml}$ of blood was collected from the second angiocath at 6 different times: just before the start of the infusion and at $0.5,1,2.5,4$, and $5 \mathrm{~min}$ after the start of infusion. The infusion was continued throughout the blood sampling. If possible, more blood was collected on the last blood draw (5min), up to $0.5 \mathrm{ml}$.

For the time after discontinuation-concentration study, approximately $0.2 \mathrm{ml}$ of blood was collected before the start of the infusion. Another $0.2 \mathrm{ml}$ of blood was collected after $5 \mathrm{~min}$ of infusion. The infusion was then discontinued and $0.2 \mathrm{ml}$ of blood was collected at $0.5 \mathrm{~min}$, $1 \mathrm{~min}, 2 \mathrm{~min}$, $5 \mathrm{~min}$ and $10 \mathrm{~min}$ following the discontinuation of the infusion. Once again, if possible, more blood was collected on the last blood draw (10min post infusion).

Blood was placed on ice until centrifugation (approximately 20min). Blood was then centrifuged at 5000RPM for $20 \mathrm{~min}$. Serum was collected and stored at $-80^{\circ} \mathrm{C}$ until lipid analysis.

\subsubsection{Lipid Analysis}

Lipid extraction and analysis were performed as described in the General Methods. One minor modification was that $0.2 \mathrm{ml}$ of serum could not always be collected due to the small amount of blood collected. Serum volume varied from 0.01 to $0.2 \mathrm{ml}$. Calculations of serum concentrations were adjusted to take into account the volume of serum involved. 


\subsubsection{Statistical Analysis}

A two-way repeated-measures ANOVA was used to evaluate the differences in blood concentration of DHA during the 5 minutes of infusion of different doses of DHA (Experiment 6A). Another two-way repeated measure was used to evaluate the difference in unesterified DHA serum concentrations following the discontinuation of the infusion of two different doses of DHA (Experiment 6B).

\subsection{Results}

The infusions used in Experiments $6 \mathrm{~A}$ and $6 \mathrm{~B}$ did not result in any mortality. The infusion of $400 \mathrm{mg} / \mathrm{kg}$ in Experiment 6A, however, resulted in the animals burying his head under the corn-cob bedding and producing rhythmic jerking behaviour. This dose was therefore dropped from the experiment.

Figure 6.1 illustrates the serum concentrations of unesterified DHA observed with different doses of DHA at different times after the start of infusion. Data are presented as means $\pm \mathrm{SD}$. As indicated, the infusion of the various doses of DHA resulted in clear-cut

increases in serum unesterified DHA concentrations. The unesterified DHA levels appeared to plateau at approximately 2.5 minutes after the start of the infusion. At the $5 \mathrm{~min}$ time point, the increases in unesterified DHA in serum followed a clear dose-concentration relationship, with higher doses producing higher concentrations. A two-way repeated measures ANOVA found a significant effect of time, dose, and an interaction between dose and time of infusion $(\mathrm{p}<0.05)$.

Figure 6.2 presents the mean $( \pm \mathrm{SD})$ serum concentrations of unesterified DHA before infusion, after 5 minutes of infusion, and at various times after the termination of infusion at 5 
Dose-Concentration Curve of Serum Unesterified DHA Concentration

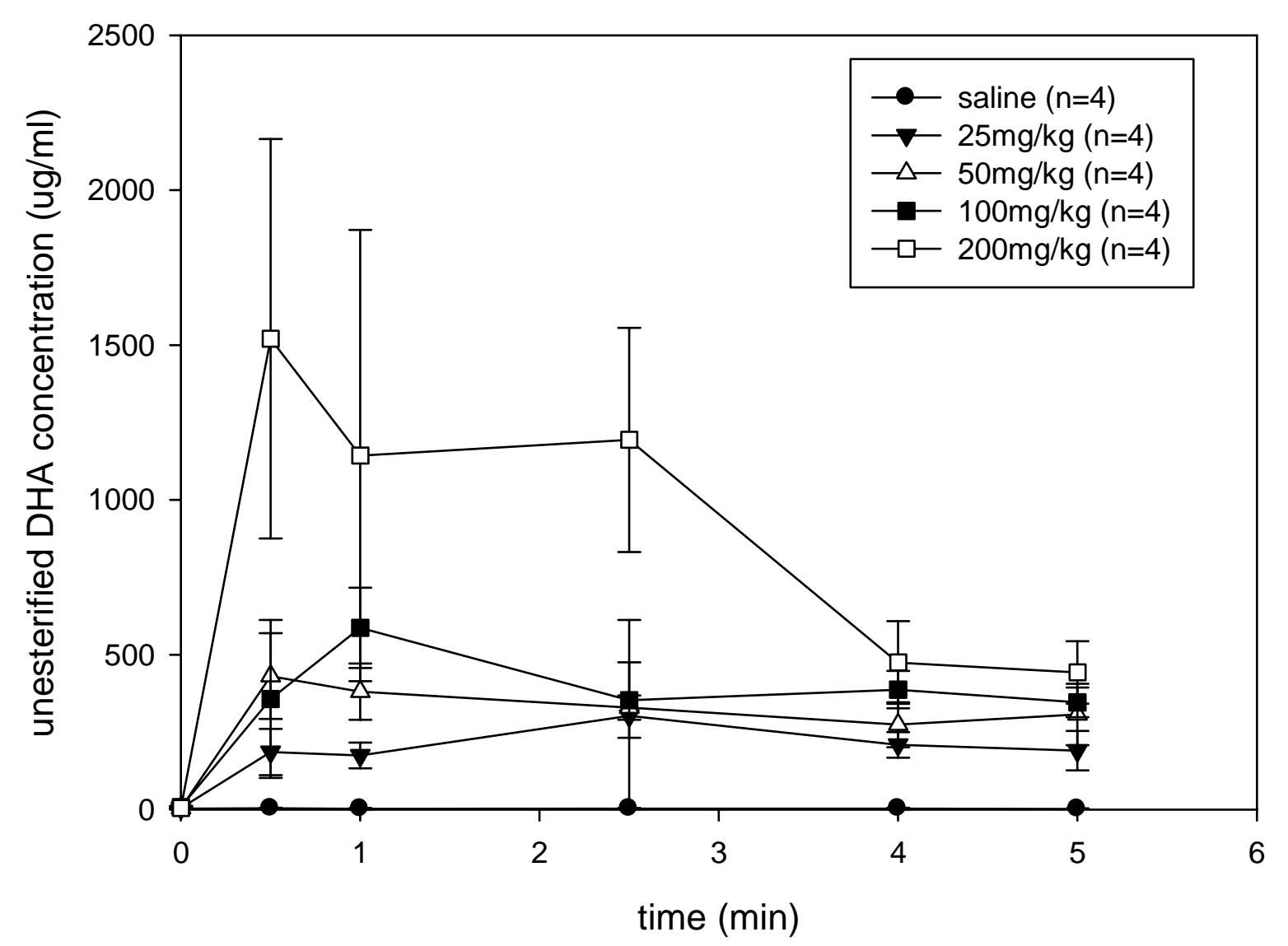

Figure 6.1. The effect of the infusion of various doses of DHA on serum unesterified DHA concentrations. Doses were infused during a period of 5 minutes and blood was collected at different times during infusion. Data are means \pm SD. A two-way repeated measures ANOVA found a significant effect of time, dose and an interaction between dose and time $(p<0.05)$. 
Rapid Disappearance of Unesterified DHA from Serum Following Discontinuation of Infusion

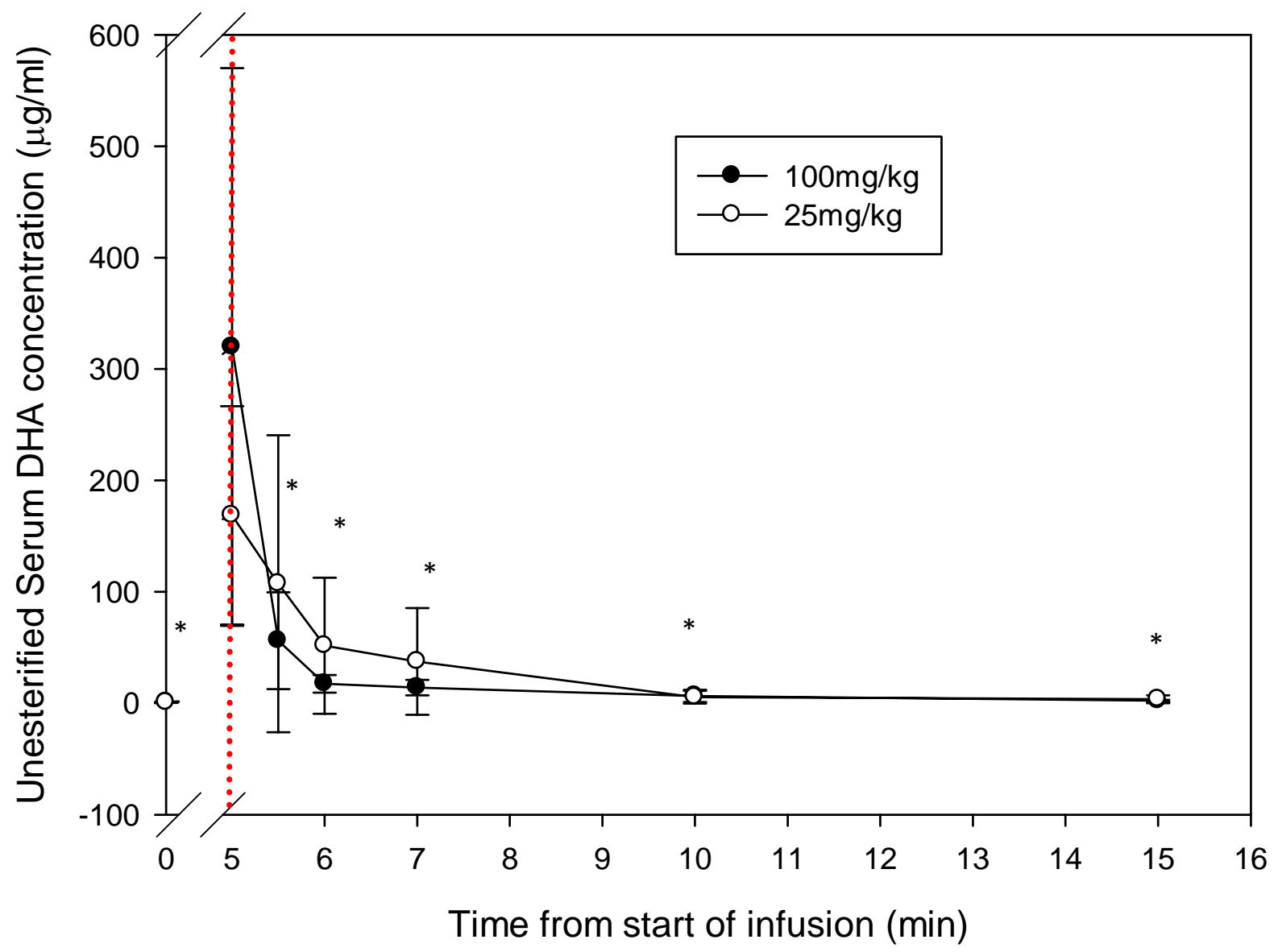

Figure 6.2. The effect of discontinuing infusion on serum unesterified DHA concentration. Averages $(n=3-4)$ are presented \pm SD. The dotted red line indicates that infusion was discontinued at the $5^{\text {th }}$ minute. After discontinuation, DHA rapidly disappeared from serum, with the half life of the 25 and $100 \mathrm{mg} / \mathrm{kg}$ dose being $34 \mathrm{sec}$ and $14 \mathrm{sec}$ respectively. Two-way repeated measures ANOVA found a significant effect of time $(p<0.05)$

*significantly different from the $5 \mathrm{~min}$ time point as indicated by Tukey's post-hoc test 
minutes. As indicated, once the infusion was discontinued, unesterified DHA levels dropped rapidly. The half lives of the 25 and $100 \mathrm{mg} / \mathrm{kg}$ doses were 34 and $14 \mathrm{sec}$ respectively. By 10 minutes after the end of the infusion, concentrations approximated $3 \mu \mathrm{g} / \mathrm{ml}$. A two-way repeated measures ANOVA found a significant effect of time $(\mathrm{p}<0.05)$. Post-hoc tests showed that the concentration of DHA in serum after $5 \mathrm{~min}$ of infusion was significantly higher than the concentration prior to infusion or the concentration at subsequent timepoints following the discontinuation of infusion (Tukey's post hoc, $\mathrm{p}<0.05$ ). It should be noted that, although not significantly elevated, serum concentrations never returned back to baseline levels, and remained approximately 3 fold higher than baseline as long as measurements were taken.

\subsection{Discussion}

DHA was administered by 5-minute infusion in the present experiments rather than by bolus injection which had caused mortality in pilot studies. The slow infusion of various doses of DHA was successfully done without any mortality.

The purpose of Experiment 6A was to evaluate serum concentrations of DHA following the infusion of DHA directly into the systemic circulation via the tail vein. It was found that 5 minutes of continuous infusion resulted in increases in serum concentration that were dose dependent.

These increases were very large, with the $200 \mathrm{mg} / \mathrm{kg}$ dose resulting in approximately a 100 fold increase as compared to baseline levels. These concentrations are extremely high, as compared to the concentrations found after our s.c. injections (Chapter 5). 
These concentrations are also high in comparison to those reported in the literature. The only previous study that measured unesterified DHA levels following i.v. infusion was done by Voskuyl and colleagues. The concentrations found in the present experiment are approximately 95 fold higher than those reported by Voskuyl et al for comparable doses (Voskuyl et al., 1998). The difference between the findings of the two studies is most likely due to the rates of infusion used. In the present experiment, $50 \mathrm{mg} / \mathrm{kg}$ was infused in $5 \mathrm{~min}$ while Voskuyl infused $40 \mathrm{mg} / \mathrm{kg}$ over 30min. Considering the short half life of DHA in serum (below), Voskuyl's slow rate of infusion probably reduced the concentrations he measured.

By measuring serum levels at different times after the start of infusion in Experiment 6A, it was possible to see concentrations rise and plateau. For most of the doses, the unesterified DHA levels appeared to plateau by 2.5 minutes after the start of the infusion. The exception was the $200 \mathrm{mg} / \mathrm{kg}$ dose where concentrations did not plateau until 5 minutes after the start of infusion. The reason for this is not clear.

These i.v. results are considerably different from those obtained by s.c. injection, and are more consistent with expectations based on the known pharmacokinetics of DHA. Increasing doses resulted in a dose-dependent increase in unesterified DHA in serum. When injected s.c., increasing doses of DHA did not result in significantly increasing serum concentrations, supporting the hypothesis that s.c. DHA may have been trapped or slowly released from the site of injection.

The purpose of the Experiment 6B was to evaluate how long DHA remained elevated within the systemic circulation following the discontinuation of DHA infusion. It was found that DHA disappeared rapidly from the serum. The half lives measured were $34 \mathrm{sec}$ and $14 \mathrm{sec}$ 
for the 25 and $100 \mathrm{mg} / \mathrm{kg}$ doses. This is in agreement with the fast half lives of other lipids in serum (Robinson et al., 1992). The fact that DHA concentrations appear to plateau within $2.5 \mathrm{~min}$ after the start of infusion is in agreement with the fast half life of $30 \mathrm{sec}$ or less, following the rule of thumb that plateau occurs at about 5 times the half life.

This is in disagreement, however, with Voskuyl's results. Voskuyl found that DHA disappeared slowly from the serum. Approximately half of the unesterified DHA was still present in the serum 30min after the discontinuation of the infusion (Voskuyl et al., 1998), which is in disagreement with known kinetics of unesterified lipids (Robinson et al., 1992). This fact could raise questions about the validity of the results reported by Voskyul.

In summary, Experiments $6 \mathrm{~A}$ and $6 \mathrm{~B}$ provided data consistent with the known kinetics of DHA and other fatty acids. They provided a solid basis for subsequent dose- and timeresponse studies (Chapter 7). 


\section{CHAPTER 7}

Dose- and Time-Response Relationship of the Anticonvulsant Effects of Infused DHA in the Maximal PTZ Seizure Model 


\subsection{Rationale}

The purpose of Experiment 7 was to explore the anticonvulsant effects of slow (5minute) infusions of various doses of DHA and to measure the disappearance of anticonvulsant effects at various times after infusion in the maximal PTZ seizure model.

Previously, in Experiment 6A, it had been shown that 5-minute infusions of DHA increased unesterified DHA in the serum in a dose-dependent manner. Experiment 6B, however, showed that this increase in serum rapidly disappeared following the discontinuation of the infusion.

Experiment 7, therefore, was designed to determine whether the dose-response and time-response relationships of DHA in the maximal PTZ seizure model paralleled the time course of the serum concentrations.

We hypothesized that a dose-response relationship would be observed, and that the anticonvulsant effects would rapidly disappear once the infusion was discontinued.

\subsection{Methods}

\subsubsection{Subjects}

Male 350g Wistar rats, similar in body weight to those in Experiment 6A, were obtained from Charles River, and were singly housed, with water and food available ad libitum as described in the General Methods section. 


\subsubsection{Drug Solutions}

DHA solution was prepared by mixing DHA and BSA in a ratio of $1.43 \mathrm{mg}$ of DHA to each mg of BSA in physiological saline.

In the dose-response experiment (Experiment 7A), the ratio was maintained for all doses by diluting the original stock solution by serial dilution to obtained the different doses in approximately $1 \mathrm{ml}$ of solution. A vehicle solution only containing BSA and saline was used in control subjects. Physiological saline only was used as a second control.

Base on results from Experiment $7 \mathrm{~A}$, the $100 \mathrm{mg} / \mathrm{kg}$ dose was used for the timeresponse experiment (Experiment 7B). A stock solution was prepared by mixing $42.4 \mu \mathrm{L}$ of DHA and $27.2 \mathrm{mg}$ of BSA per $\mathrm{ml}$ of physiological saline in order to obtain a $100 \mathrm{mg} / \mathrm{kg}$ concentration in approximately $1 \mathrm{ml}$ of volume. A vehicle solution containing only $27.2 \mathrm{mg}$ of BSA and saline was used as control. Solutions were stored as previously described.

\subsubsection{Drug Infusions}

Subjects were placed under a heat lamp for 20 minutes to cause vasodilation. A single 24 gauge angiocath was placed in the right tail vein, and an infusion line was connected to the angiocath.

In Experiment 7A, each subject received either 0 (saline or saline with BSA), 12.5, 25, 50,100 , or $200 \mathrm{mg} / \mathrm{kg}$ of DHA over 5 minutes in approximately $1 \mathrm{ml}$ of solution (Volume of injection varied slightly depending on weight). 
In Experiment $7 \mathrm{~B}$, each animal received either $0(\mathrm{BSA})$ or $100 \mathrm{mg} / \mathrm{kg}$ of DHA in approximately $1.3 \mathrm{ml}$ of solution over the course of 5 minutes. (The volume of injection varied slightly depending on weight.) The $100 \mathrm{mg} / \mathrm{kg}$ dose was chosen because more subjects showed seizure suppression at this dose in Experiment 7A.

\subsubsection{Seizure Testing}

In Experiment 7A, following 5 minutes of infusion, subjects received $100 \mathrm{mg} / \mathrm{kg}$ of PTZ i.p. (dose determined by pilot data in this group of subjects). The DHA infusion was continued for an additional 5 minutes after the PTZ injection (total of 10 minutes of infusion) in order to maintain a steady concentration of unesterified DHA in serum during the period of seizure testing. Latency to tonic-clonic seizures was measured.

In Experiment 7B, subjects received $110 \mathrm{mg} / \mathrm{kg}$ of PTZ i.p. (dose determined by pilot data) at varying times before and after the end of the DHA infusion. Times - as measured from the start of infusion - were: $4 \mathrm{~min}$ (during the infusion), 5min (right at the end of the infusion), 7.5 (2.5min post infusion), 10min (5min post infusion, and $15 \mathrm{~min}$ (10min post infusion). Latency to the onset of tonic-clonic seizures was measured.

\subsubsection{Statistical Analysis}

In Experiment 7A, outlier analysis was done using chi square analysis. Chi Square analysis revealed a trend $(\mathrm{p}<0.06)$ towards a difference between groups for outliers, but the difference was not significant. Outliers were therefore kept in the analysis. 
A one-way ANOVA was used to analyze the differences in seizure latency between the different doses of DHA. Due to the great variability, a non-parametric Chi square test was also performed. Subjects that did not seize within 5 minutes after the PTZ injection $(25 \mathrm{mg} / \mathrm{kg} \mathrm{n}=2$, $100 \mathrm{mg} / \mathrm{kg} \mathrm{n}=3,200 \mathrm{mg} / \mathrm{kg} \mathrm{n}=2$ ) were assigned an arbitrary time score of $300 \mathrm{sec}$ - since this was the time at which the DHA infusion was terminated.

In Experiment 7B, outlier analysis was done using chi square analysis. Outlier analysis was not significant, therefore outliers were removed from analysis (saline $n=1,4 \mathrm{~min} n=2,5 \mathrm{~min}$ $\mathrm{n}=1,15 \mathrm{~min}=1$ ). A Chi square analysis was performed to analyze the differences between groups, since the violated the assumptions for parametric tests.

\subsection{Results}

Figure 7.1 presents the mean $( \pm \mathrm{SD})$ latencies to tonic-clonic seizures for the infusion of the different doses of DHA (Experiment 7A). Figure 7.2 illustrates the great variability of the latencies to tonic-clonic seizures of individual subjects in a scatter plot. The slow infusion of DHA was anticonvulsant against tonic-clonic seizures in the maximal PTZ model. Saline and BSA were not statistically different from one another $(\mathrm{p}>0.05)$, however, and were therefore combined. A significant overall effect of DHA infusion was found as indicated by one-way ANOVA $(\mathrm{p}<0.05)$. Tukey's post-hoc tests did not reveal significant difference between any two pairs of means. The highest latency to tonic-clonic seizure for the control group was 66sec. A secondary Chi square analysis also found a significant difference between the groups for animals seizing before or after $66 \mathrm{sec}$, the longest latency found in the control group $(\mathrm{p}<0.05)$. 
Slow DHA Infusion Dose-Response Curve in the Maximal PTZ Model

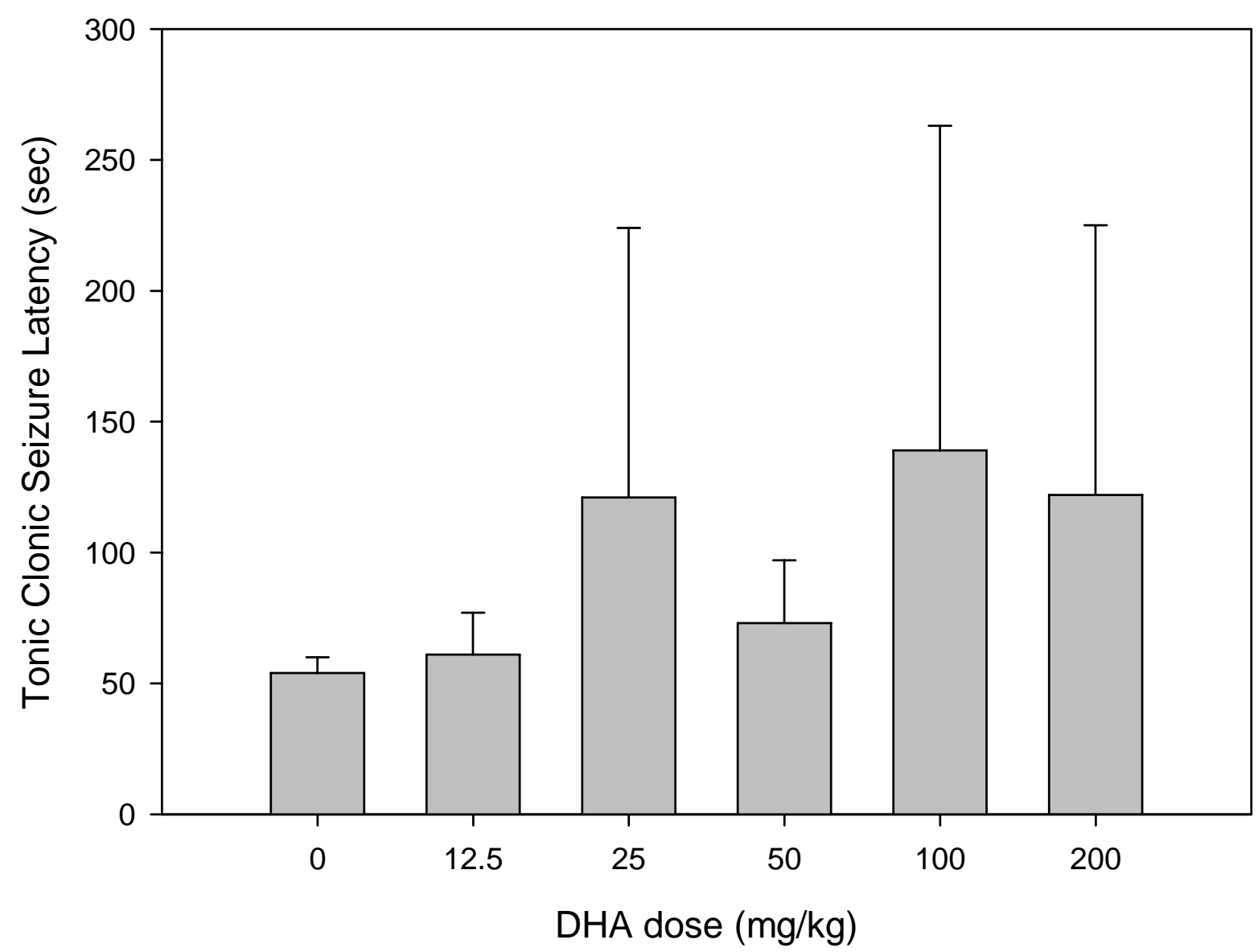

Figure 7.1. Latency to onset of PTZ-induced tonic-clonic seizures following 5min of i.v. infusion of different doses of DHA. Data is presented as mean $\pm \mathrm{SD}(\mathrm{n}=8-14$ per group). A one-way ANOVA found an overall significant effect of DHA infusion. 
Slow DHA Infusion Dose-Response Curve in the Maximal PTZ Model (Scatter Plot)

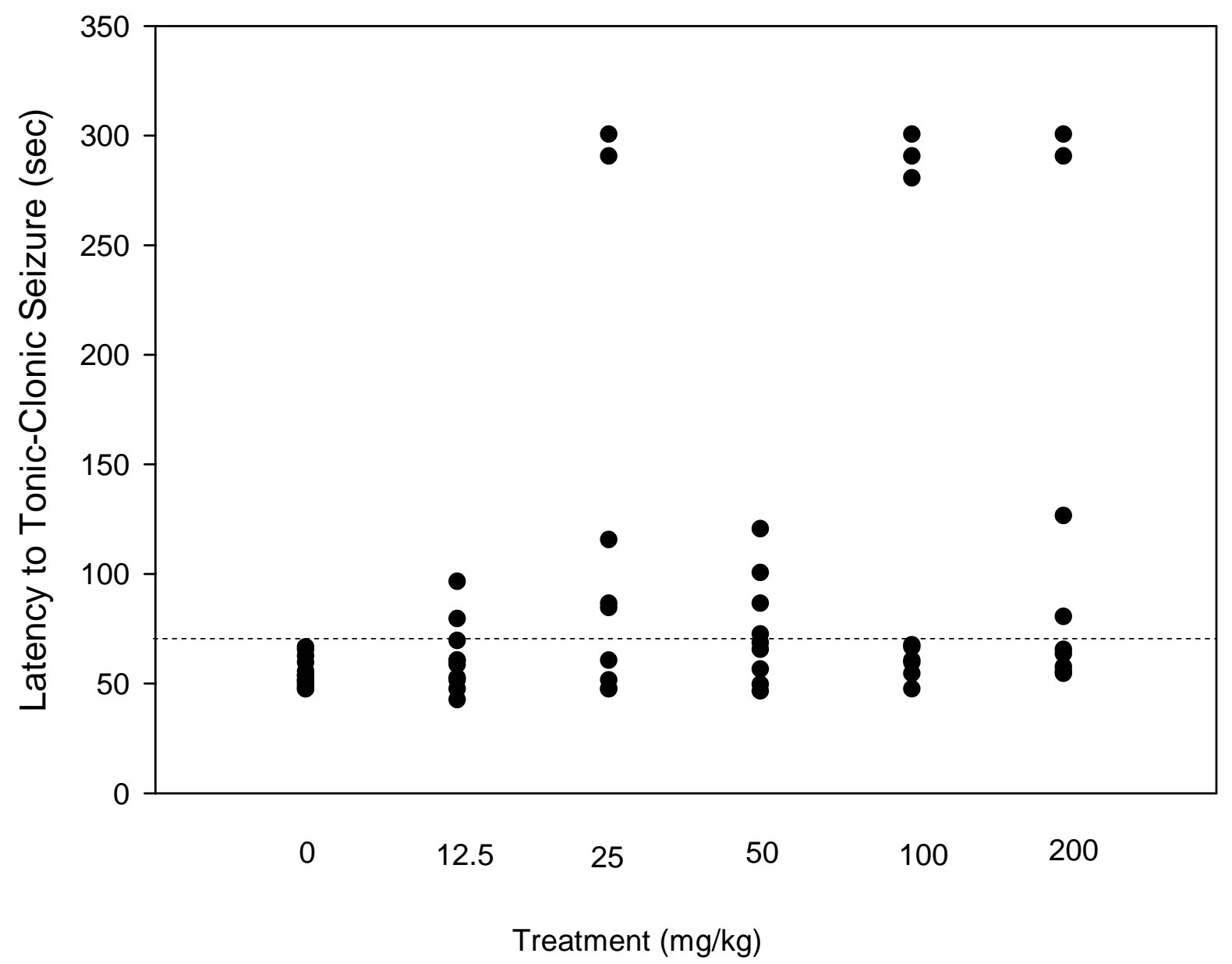

Figure 7.2. Latency to onset of PTZ-induced tonic-clonic seizures following $5 \mathrm{~min}$ of i.v. infusion of different doses of DHA presented as a scatter plot. The dotted line represents the $66 \mathrm{sec}$ timepoint, the longest latency in the control group. Each DHA group had several subject over this threshold (12.5n=3, $25 n=5,50 n=5,100 n=4,200 n=4)$ 
Figure 7.3 presents the mean latencies (+/- SD) to tonic-clonic seizures for the different treatment groups after discontinuation of infusion. As shown in Figure 7.3, latencies in the DHA groups were increased as compared to the saline-albumin control, and the effects appeared to be long lasting, with seizure threshold still elevated $15 \mathrm{~min}$ after the start of infusion. The longest latency to tonic-clonic seizure in the control group was 60sec. Chi square analysis revealed a significant difference between groups for animals seizing before and after $60 \sec (\mathrm{p}<0.05)$. There did not appear to be any difference between DHA groups.

Figure 7.4 presents the latencies of individual rats in a scatter plot. As indicated by Figure 7.4, there was a great deal of variability in the treatment groups, whereas the saline control group had relatively low variability.

\subsection{Discussion}

In Chapter 6, it was found that unesterified DHA increased in a dose-dependent manner. It was also found that this increase in serum DHA disappeared rapidly once infusion was discontinued. Experiment 7A, therefore, was designed to evaluate whether the dosedependent increase in unesterified DHA would correlate with a dose-dependent increase in seizure protection. Experiment 7B was designed to evaluate whether the rapid disappearance of serum DHA would correlate with a disappearance of anticonvulsant effects.

In Experiment 7A, it was found that slow (5-minute) infusions of DHA increased seizure protection in the maximal PTZ model. This protection appeared at a dose of $25 \mathrm{mg} / \mathrm{kg}$, and did not increase at higher doses, since subjects in the $25 \mathrm{mg} / \mathrm{kg}$, the $100 \mathrm{mg} / \mathrm{kg}$ and the $200 \mathrm{mg} / \mathrm{kg}$ groups all had similar mean latencies. 
Time-Response Curve for DHA Infusion in the Maximal PTZ Model

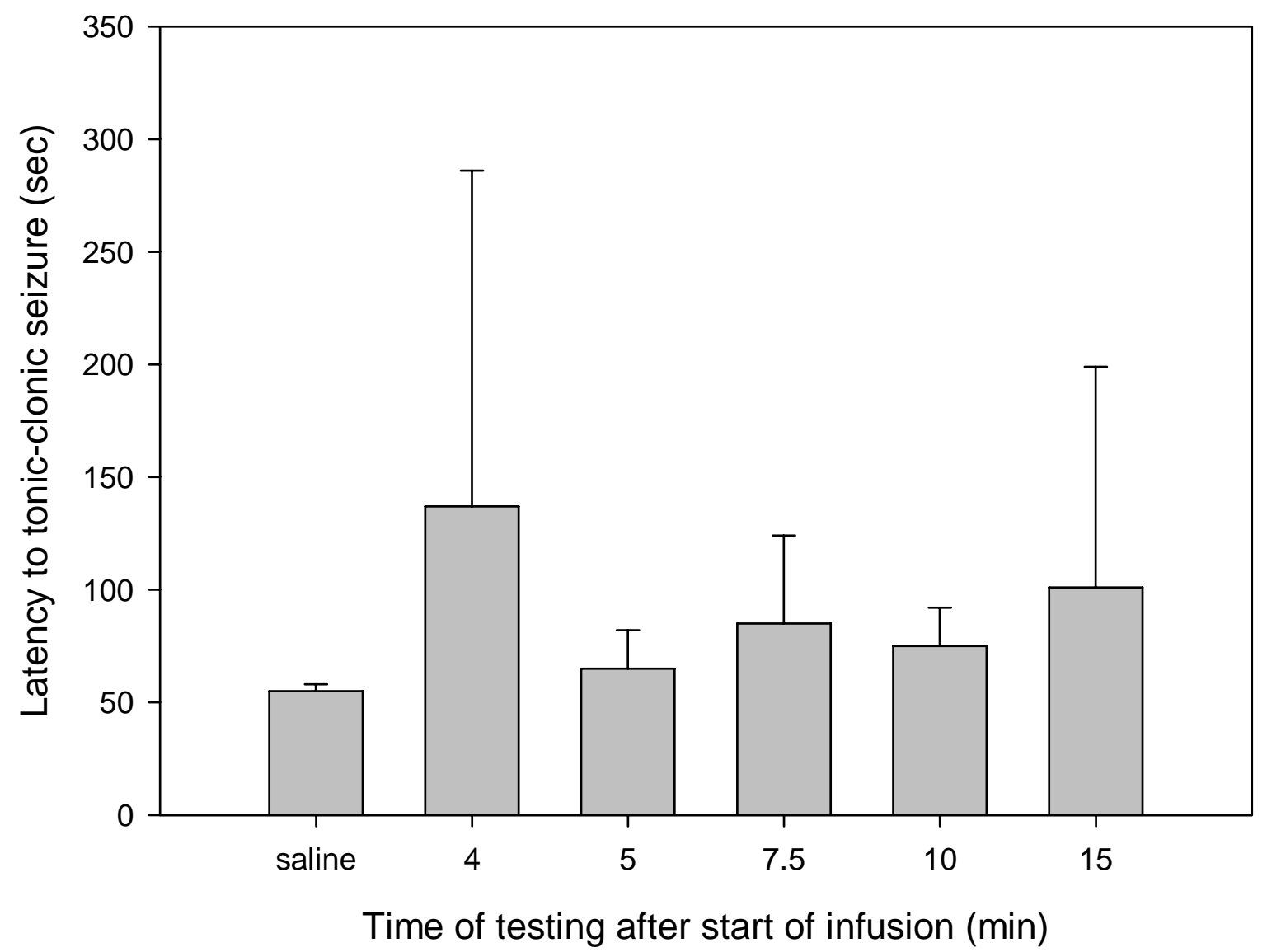

Figure 7.3. Effect of time on latency to the onset of tonic-clonic seizures. Infusion was discontinued after $5 \mathrm{~min}$ of infusion. Averages $(\mathrm{n}=7-9)$ are presented $\pm \mathrm{SD}$. Chi square analysis reveals a significant difference between groups for animals seizing before and after 60sec post PTZ injection. 
Time-Response Curve for DHA Infusion in the Maximal PTZ Model (Scatter Plot)

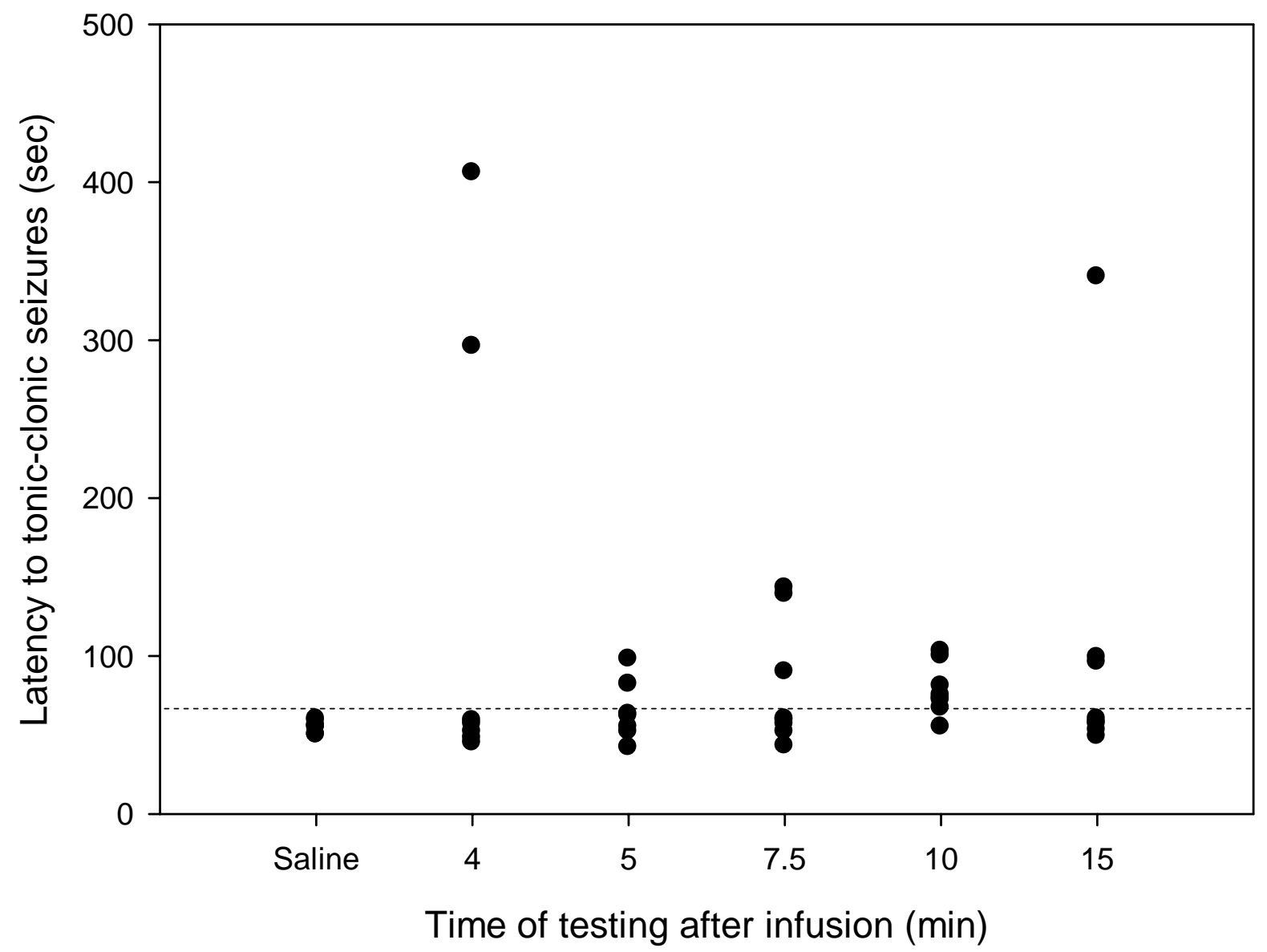

Figure 7.4. Effect of time on latency to tonic-clonic seizures (scatter plot). Infusion was discontinued after $5 \mathrm{~min}$ of infusion. The dotted line indicates the $60 \mathrm{sec}$ timepoint, the longest latency in the control group. Each experimental group had several subject seize past this timepoint $(4 \min n=2,5 \min n=4,7.5 \min n=3,10 \min n=7,15 \min n=3)$ 
Actual seizure suppression was observed for the first time in Experiment 7A, since in some subjects no seizure activity was seen within 5 minutes after the PTZ injection (at which time the DHA infusion was stopped). Once again, there did not seem to be an increase in effect with doses above $25 \mathrm{mg} / \mathrm{kg}$. Seizure suppression was seen in 2 animals in the $25 \mathrm{mg} / \mathrm{kg}$ group and also in 2 animals in the $200 \mathrm{mg} / \mathrm{kg}$ group.

It is possible that seizure suppression was seen in the present study because DHA levels in the blood were higher than they had been in our s.c. studies. As indicated in Experiment 6A, with i.v. infusions we were able to increase serum levels to $50-500 \mu \mathrm{g} / \mathrm{ml}$ or approximately 400 fold of baseline. The highest concentrations achieved in our s.c. studies had been $0.6 \mu \mathrm{g} / \mathrm{ml}$. It is possible that these high levels also explain the fact that there was no increase in effect at the higher doses of DHA. We may have reached a "ceiling" at $25 \mathrm{mg} / \mathrm{kg}$.

Although some seizure suppression was seen in Experiment 7, our results still stand in apparent contrast to the previous results reported by Voskuyl et al. (1998). Voskuyl reported a great increase in electrical threshold at serum levels of approximately $3.2 \mu \mathrm{g} / \mathrm{ml}$. Although he didn't actually measure seizure suppression, one would expect that this great increase in threshold would have resulted in seizure suppression in most subjects. This differs from our results, where the most suppression we saw was approximately $40 \%$ despite the fact that our serum levels were far higher than Voskuyl's.

It is possible that this difference in results could be due to the difference in models. The maximal PTZ model measures seizure suppression, whereas the cortical stimulation model used by Voskuyl measures seizure threshold. It is possible that the stimulus in our model may have been stronger than Voskuyl, and therefore that we may have failed to detect subtle effects. 
Voskuyl made use of a model that generated multiple afterdischarges per day. It is known that afterdischarges causes inhibition and results in an increase in ADT in subsequent stimulations (Mucha and Pinel, 1977). It is therefore possible that his increase in seizure threshold is an artefact caused by his model. It should be noted that Voskuyl also observed increases in seizure threshold with the treatment of OA. In our hands, and in vitro, OA has yet to be shown to have any anticonvulsant effects. This may be a result of multiple afterdischarges increasing ADT. It should be noted, however, that Voskuyl did not report any increase in threshold in their vehicle group.

It is possible, however, that our infusions may not have lasted long enough to be fully effective. It is interesting to note in Figure 7.2 (the scatter plot) that while some subjects showed complete seizure suppression at the higher doses, other subjects appeared to show no effect at all. This might indicate that our infusions were long enough to affect some subjects, but not all subjects. Voskuyl et al found their effect following 30 minutes of infusion (Voskuyl et al., 1998) (Voskuyl's dose, delivered over 30 minutes, was 40mg/kg which was similar to our $50 \mathrm{mg} / \mathrm{kg}$ dose given in only 5 minutes). We cannot rule out the possibility that longer exposure times might be more effective. This could be a topic for future experiments.

The variability in seizure latency could also be explained by the fasting status of the animals. It is known that fasting prior to the start of the ketogenic diet may result in a reduction in seizure in kids (Zupec-Kania and Spellman, 2008). It is therefore possible that rats may have higher seizure threshold depending on the time meal. If this was the case, however, all groups including control animals, would have been affected, which was not observed. Control animals showed very little variation. Moreover, animals had access to food right until seizure testing. 
In one respect, however, our results are in clear disagreement with the previous findings of Voskuyl et al. Voskuyl et al. saw no anticonvulsant effects until they had infused DHA for 30 minutes. In contrast, we saw clear effects after 5 minutes of infusion, even with doses lower than the $40 \mathrm{mg} / \mathrm{kg}$ that Voskuyl used. The reasons for this difference are not clear, although different seizure models were used. It should be noted, however, that past studies have shown that fatty acids rapidly (within seconds) enter the brain (Chen et al., 2009; Ouellet et al., 2009). It might therefore be expected DHA would enter the brain and have antiepileptic effects within the 5 minutes of infusion.

In Experiment 6B, we found that DHA was rapidly cleared from serum and completely disappeared within 5min of the discontinuation of the DHA infusion. One might expect a similar rapid decrease in anticonvulsant effects would occur following the discontinuation of a DHA infusion. We hypothesized that they would. Voskuyl et al. (1998), however, had reported that their maximal anticonvulsant effects were seen 6.5 hours after the end of their infusions, when serum unesterified DHA levels had gone back to baseline levels.

In Experiment 7B, contrary to our hypothesis, it was found that anticonvulsant effects appeared to continue even after serum levels of unesterified DHA must have dropped to baseline. Even 10 minutes after the discontinuation of the infusions, latency to tonic-clonic seizure appeared to be elevated. The results are in general agreement with Voskuyl et al., who found that seizure threshold were still elevated hours following the discontinuation of the infusion (Voskuyl et al., 1998).

Voskuyl et al. suggested that these long-lasting anticonvulsant effects might be mediated by the esterified forms of DHA, which could still be elevated after serum unesterified 
DHA levels had dropped back to baseline. This suggestion, however, is not in agreement with the electrophysiological studies that show that it is the unesterified form of DHA that has antiarrhythmic/anticonvulsant activity (Kang and Leaf, 1994, 1996; Xiao and Li, 1999).

The results of Experiment $6 \mathrm{~B}$ suggest another possibility. In Experiment 6B, it was shown that unesterified DHA levels dropped to near baseline levels within 30 seconds. Though levels dropped to near baseline, however, they never actually returned to baseline. Although not significantly elevated, unesterified DHA concentrations were still approximately 3 fold higher than baseline 10 minutes after the discontinuation of DHA infusion. This small increase in serum unesterified DHA concentrations will have to be verified in future experiments using larger numbers of subjects. If it can be verified, however, it could possibly explain why anticonvulsant effects were still present 10 minutes after the discontinuation of the infusion.

If it is in fact true that DHA remains elevated after the discontinuation of 5 minutes of DHA infusion, it is possible that this could be due to a shift in equilibrium. When in serum, DHA rapidly disappears to enter other tissues, including adipose, heart, muscle and brain. DHA is then esterified within the tissue. Other DHA molecules, however, are released through lipolysis and can leak back into the circulation, maintaining an equilibrium between tissue and circulation (Mayes and Botham, 2003). It is possible that our infusion could have entered the tissue and then been sequestered into the phospholipid membranes, which would explain the rapid disappearance in the blood. It is also conceivable, however, that due to the high amount of DHA infused, the equilibrium between the tissue and the circulation may have been shifted, providing a possible explanation for the unesterified DHA never returning to baseline levels and remaining slightly elevated after our infusions. 


\section{CHAPTER 8}

General Discussion 


\subsection{Major Findings}

The purpose of the present research was to test the anticonvulsant effects of the n-3 PUFAs, and more specifically DHA, in vivo. The idea that the n-3 PUFAs might be anticonvulsant was based on their antiarrhythmic effects, which occur due to their modulation of the VDSC. Compounds that are antiarrhythmics may also be anticonvulsant.

The idea that n-3 PUFAs might be anticonvulsant had been tested by previous work by our group (Taha, 2009). The results of these previous studies were promising; however, they raised multiple unsolved questions. The present thesis attempted to address these questions in the hopes of providing further evidence of the n-3 PUFAs' anticonvulsant properties.

In general, the present research has suggested that the n-3 PUFAs, and more specifically DHA, have anticonvulsant properties. The results from individual experiments are summarized in table 8.1. Administration of n-3 PUFAs - and specifically DHA - resulted in an increase in threshold in rats, whether the PUFAs were given chronically, sub-chronically or acutely.

\subsubsection{Chronic Administration}

Experiment 3, for instance, investigated the effects of the chronic administration of fish oil, a rich source of n-3 PUFAs, on amygdala ADTs in an electrical stimulation model. Chronic fish oil administration raised amygdala ADTs, although this increase did not appear until the third month of administration (Discussion of the time course appears below). 


\subsubsection{Sub-Chronic Administration}

In Experiment 4, the anticonvulsant properties of sub-chronic administration of DHA over 14 days were evaluated. This study attempted to replicate a previous study that had used DHA, the most abundant n-3 PUFA in the brain, so that DHA rather than fish oil was administered. I.p. injections of DHA and ethyl ester DHA over a 14 day period resulted in an increase in seizure protection in the maximal PTZ model, although we did not see the dramatic seizure suppression previously reported with this technique (Bruzzese, 2005).

\subsubsection{Acute Administration}

Experiments 5-7 involved acute administration of DHA. Previously, in a small pilot study, our group had found that acute s.c. administration of DHA resulted in an inverted-U dose-response curve. Inverted-U curves are rarely seen in pharmacology. Experiment 5, therefore, was designed to confirm this inverted-U dose-response curve. In Experiment 5, as found in our previous work, acute s.c. injections of DHA were protective in the maximal PTZ model. This effect, however, was seen at lower doses and lost with higher doses, confirming the inverted-U dose previously reported (Taha, 2009).

We hypothesized that this inverted-U dose-response curve might be due to the fact that DHA was being injected via the s.c. route. We hypothesized that DHA might be coming out of solution at the site of injection, with larger concentrations showing this effect more strongly than lower concentrations. We therefore tried administering DHA via i.v. injections through 
the tail vein. Since pilot studies had showed that bolus injections could be lethal, we slowly infused intravenously over a period of 5 minutes.

Our first i.v. study was a dose-serum concentration study. In Experiment 6, therefore, unesterified DHA concentrations were measured following slow DHA infusion. Serum concentration increased rapidly in a dose-dependent manner, reaching plateau approximately 2.5 minutes after the start of infusion. Concentrations dropped to near baseline within a minute following the discontinuation of DHA infusion.

In Experiment 7, following the success of Experiment 6, we performed dose- and timeresponse studies in the PTZ model. As predicted by our dose-serum concentration studies, we found anticonvulsant effects within the first few minutes of administration, and these appeared to be dose-related. For the first time, we achieved seizure suppression in a small percentage of animals. Surprisingly, and contrary to expectations, the anticonvulsant effects of i.v. infusions tended to persist even after DHA levels had dropped in the serum.

These curious time-response data might be the subject of future experiments. Both our dose-response and our time-response data showed a great deal of variability, however, and firm conclusions should not be drawn until the experimental results have been replicated. The variability itself should be the topic of further investigations.

The data from the present experiments have solved some of the questions raised by the previous work in our laboratory. We have shown, for instance, that chronic administration does take months to take effects and that the increase in amygdala threshold occurs as quickly as the increase in the cortex when an adequate dose is administered. We have also demonstrated that s.c. injection of DHA does result in an inverted $U$ dose response curve, but 
that a normal dose-response curve is observed when DHA is given i.v., suggesting that the inverted $U$ dose- response curve observed with s.c. injections is due to pharmacokinetic issues rather than pharmacodynamic issues.

Table 8.1: List of Experiments and Results

\begin{tabular}{|c|c|}
\hline Experiment 3 & $\begin{array}{l}\text { - Chronic fish oil administration increased amygdala ADT } \\
\text { - Effects only appeared following } 3 \text { months of supplementation }\end{array}$ \\
\hline Experiment 4 & $\begin{array}{l}\text { - Sub-Chronic administration of DHA for a } 14 \text { day period } \\
\text { increased seizure protection in the maximal PTZ model }\end{array}$ \\
\hline Experiment 5 & $\begin{array}{l}\text { - Acute s.c. DHA administration was protective in the maximal } \\
\text { PTZ model } \\
\text { - Effects were lost at higher doses } \\
\text { - } \begin{array}{l}\text { s.c. DHA increases unesterified DHA in serum. Ineffective } \\
\text { dose also increased unesterified DHA concentrations in serum }\end{array}\end{array}$ \\
\hline Experiment 6 & $\begin{array}{l}\text { - } \begin{array}{l}\text { Dose-dependent increase in unesterified DHA in serum during } \\
\text { slow i.v, DHA infusion }\end{array} \\
\text { - Serum concentration reached a plateau after approximately } \\
2.5 \text { min of constant infusion } \\
\text { - Serum concentration dropped rapidly following } \\
\text { discontinuation of infusion }\end{array}$ \\
\hline Experiment 7 & $\begin{array}{l}\text { - Slow DHA i.v. infusion resulted in seizure protection in the } \\
\text { maximal PTZ model } \\
\text { - Higher doses appeared to be more protective } \\
\text { - Effects appeared to be long lasting }\end{array}$ \\
\hline
\end{tabular}

\subsection{Theoretical Model}

The present work has also raised some new questions. We confirmed that chronic oral feeding of fish oil resulted in an increase in seizure threshold after months of administration. 
When given acutely, however, we observed anticonvulsant effects within 4 minutes of administration. This raises the question why such a difference in the onset of anticonvulsant action exists between chronic oral feeding and acute systemic injections of n-3 PUFAs.

This question can best be discussed in the context of a full understanding of how PUFAs enter the body and proceed to the brain. Figure 8.1 illustrates the progress of n-3 PUFAs from the stomach to the liver and adipose until its incorporation into the brain. It is in the brain where n-3 PUFAs most probably act to modulate ion currents to increase seizure threshold. The figure assumes that the PUFAs have been ingested orally.

\subsubsection{FFAs in the Body}

Once fats - triglycerides - are ingested, they are broken down in the intestine into monoglycerides and FFAs by bile salts and lipases. Monoglycerides and FFAS are then absorbed into the enterocytes - the cells of the intestinal wall. In the enterocytes, the monoglycerides and FFAs are packaged into CM (chylomicrons) - mainly esterified triglycerides associated with lipoproteins (Mansbach and Siddiqi, 2010).

Once packaged, the CM passes through the internal wall of the enterocyte and enters the lymphatic system. From there it drains into the brachiocephalic vein, passes through the heart, and enters the systemic circulation.

In the systemic circulation, lipoprotein lipases located on the endothelial wall cleave

FFAs off the CM (Mead, 1963; Karpe et al., 2007). Most of the FFAs released from the original $\mathrm{CM}$ are incorporated into peripheral tissue, mainly the adipose, muscle, and heart 
(Bragdon and Gordon, 1958; Rodbell, 1960; Mayes and Botham, 2003). Some FFAs that are released into circulation are bound to albumin, however, and therefore can be incorporated into the brain.

The remaining CMs, also known as the "CM remnant", go on to the liver. The liver repackages the CM remnant into very low density lipoprotein (VLDL). Lipoprotein lipases then cleave off some FFAs which enter the extrahepatic tissue (Garfinkel et al., 1967). The remaining VLDL becomes a low density lipoprotein (LDL). Approximately $30 \%$ of LDL is taken up into tissue through the LDL receptor. The remaining $70 \%$ of LDL is taken up by are liver (Mayes and Botham, 2003).

Thus, the majority of ingested lipids end up in esterified form, either in the blood as lipoproteins or in adipose tissue. The adipose tissue, in particular, is the biggest lipid reservoir. The adipose reservoir is in equilibrium with a small pool of unesterified fatty acids in the blood which are bound to albumin (As discussed below, only unesterified FFAs can enter the brain).

In the adipose tissue, lypolysis and esterification are continuously ongoing in order to maintain a balance of free and esterified lipid in the adipose tissue, and indirectly blood levels (Mayes and Botham, 2003). Thus, the adipose tissue - the largest reservoir of lipids - is also thought to be the biggest source of FFAs for plasma (Fredrickson and Gordon, 1958).

Relevant to the present thesis, if chronic feeding of fish oil increases esterified n-3 PUFAs in adipose tissue, a secondary increase in serum unesterified n-3 PUFAs should also occur. This increase, however, might take some time to be achieved, as the half life of lipids in adipose tissue is long. The half life of unsaturated fatty acids in adipose tissue in rats, for instance, is approximately 20 days (Pihl et al., 1950). This would mean that half of the fatty 
acids taken up into adipose tissue on day 1 of chronic feeding would still be present in the adipose tissue 20 days later. Thus, there would be a quick incorporation of ingested lipids into the adipose tissue, and, following that, a slow release of FFAs into the blood.

The half life in human adipose tissue has been estimated at approximately 300 days (Hirsch et al., 1960). Therefore, if anticonvulsant effects depend on FFAs in the blood, one could assume that anticonvulsant effects would take even longer to appear in humans than in rats.

\subsubsection{FFAs in the Brain}

The increase in FFAs in the blood is of importance, since - as noted above - the FFA pool is thought to be the only lipid pool available to the brain (Hamilton et al., 2001). FFAs are able to detach themselves from albumin and passively diffuse into the brain (Ouellet et al., 2009). Lipoprotein receptors/transporters do exist in the blood-brain barrier, and these can actively transport FFAs. Knocking out the brain lipoprotein receptors, however, does affect fatty acid concentration in the brain (Song et al., 2010). Transport of FFAs seems to be a passive process.

Although all PUFAs can passively diffuse into the brain, incorporation into brain tissue is quite selective. DHA and AA are abundantly found in the brain, whereas EPA, LA and ALA are found in negligible amounts. This can be explained by the different rates of beta-oxidation of PUFAs in the brain. EPA, ALA and LA are selectively beta-oxidized (Demar et al., 2005; 
DeMar et al., 2006a; Chen et al., 2009), whereas DHA and AA are selectively incorporated into the phospholipid pool(Chen et al., 2009).

Once taken up into the brain, n-3 PUFAs can have functional effects. They modulate ion channels and currents, and can reduce both sodium and calcium currents in the CA1 region of the hippocampus (Vreugdenhil et al., 1996). More recently, it has been reported that n-3 PUFA also have the ability to modulate the Shaker $\mathrm{K}$ channel, increasing the potassium conductance (Xu et al., 2008). They also increase the stimulation threshold required to initiate an action potential (Xiao and $\mathrm{Li}, 1999$ ), and are therefore able to decrease frequency of neuronal firing (Young et al., 2000).

Since only DHA and AA enter the brain without being beta oxidized upon entry (Demar et al., 2005; Chen et al., 2009), it is hypothesized that it is DHA which raises seizure thresholds. Theoretically, it might be free or phospholipid DHA which has these effects.

Most studies suggest, however, that it is the free form of DHA that is functionally active and which increases seizure threshold. While n-3 PUFAs are able to modulate sodium currents in both cardiomyocytes and neurons, only the unesterified form, however, has the ability to directly bind to VDSC in cardiomyocytes.

\subsubsection{Chronic Oral Administration vs. Acute Systemic Administration}

From this theoretical model, the question related to the difference in time to onset of action between chronic oral and acute systemic administration can be answered. 
When taken orally, fats are rearranged into CM in the enterocytes. From there, they are either taken up by the tissues, mostly adipose tissue, or go to the liver to be rearranged into other VLDL. Only FFAs bound to albumin, however, can be utilized for brain uptake. An increase in serum FFAs is not seen immediately as lipids are transferred from CM and VLDL to adipose. The half life of fatty acids in adipose tissue is approximately 20 days. Therefore, only half of the ingested fat on the first day of treatment would have left the adipose tissue, as FFAs. An increase in serum FFAs, and more importantly DHA, would take months to occur following the start of oral intake. This would suggest that a long period of time would be required to increase the amount of free DHA able to reach the brain.

When administered acutely, either s.c. or i.v., however, FFAs are not repackaged into $\mathrm{CM}$ as they do not pass through the stomach's enterocytes. Therefore, DHA enters the systemic circulation as FFAs, and is immediately available for brain uptake. As it can enter the brain immediately, injected DHA can have effects within minutes.

\subsection{Clinical Relevance}

The clinical relevance of the present research is that it suggests that n-3 PUFAs might be used clinically to treat people with seizure disorders.

The current AED medication comes at a high cost - particularly if the drugs are newly released - and with multiple side effects. n-3 PUFAs could potentially offer a cheap therapy alternative, with minimal side effects. 
Figure 8.1: Fate of PUFAs Following Oral Intake
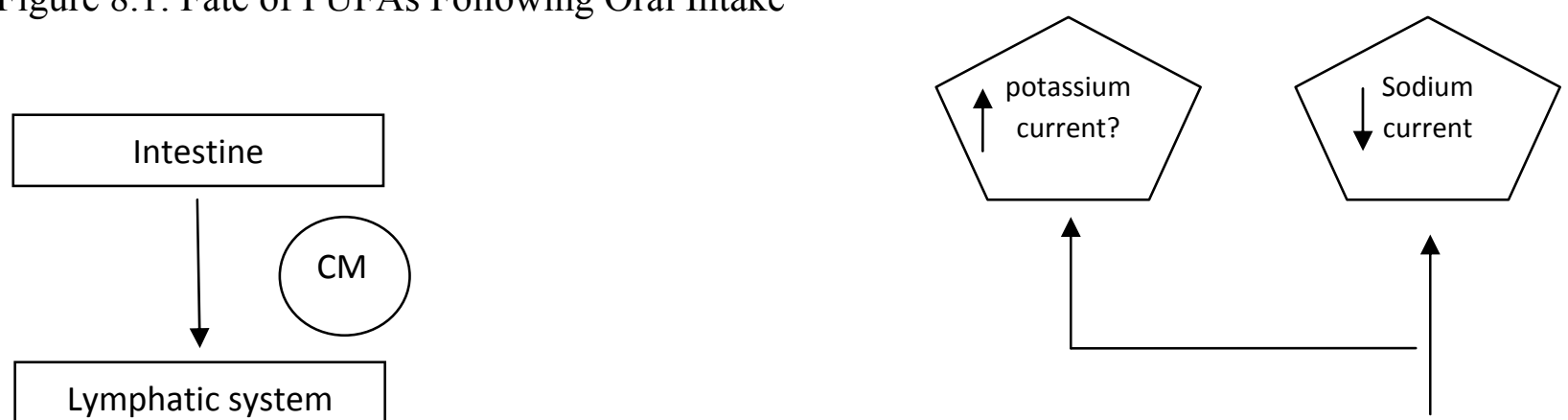

Lymphatic system

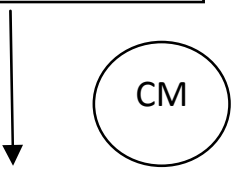

Systemic circulation

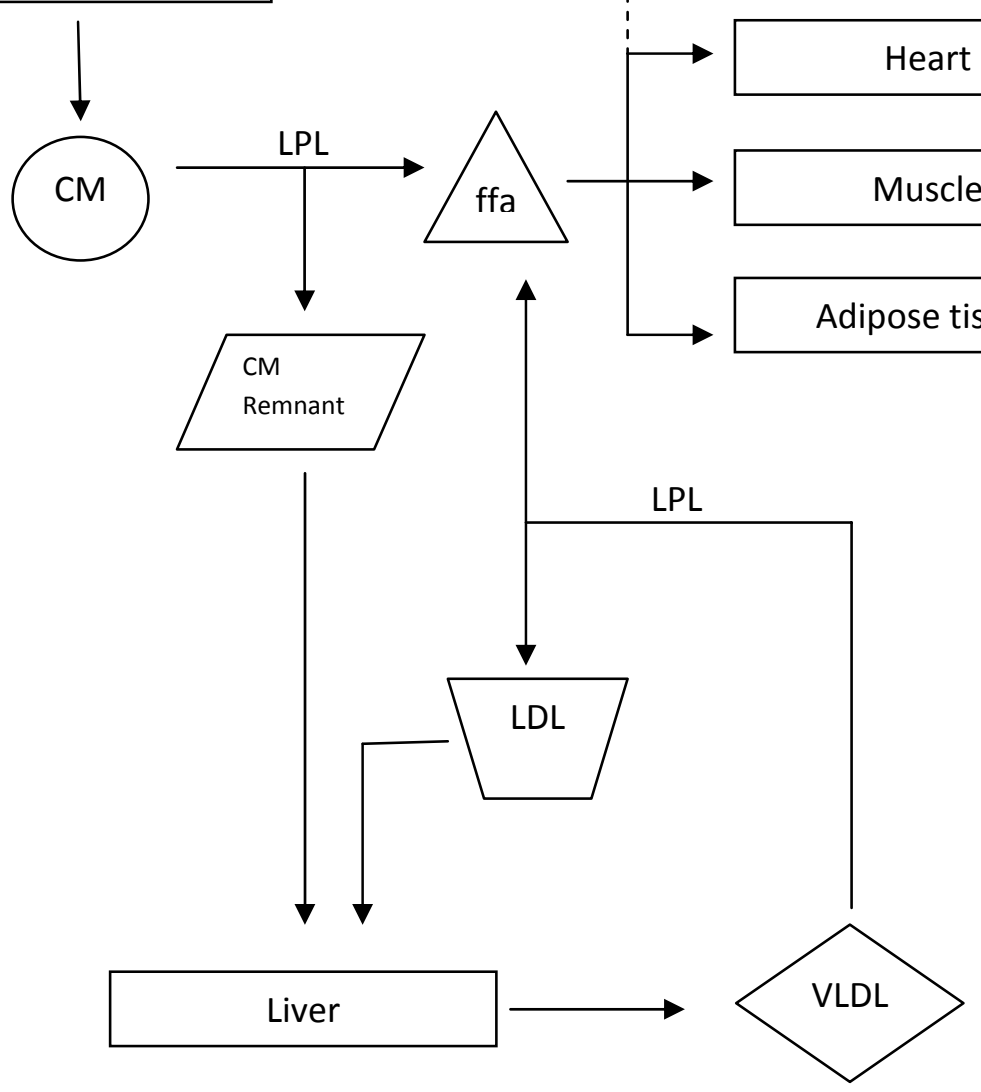

\section{Indicates minor pathway}


Further clinical trials, however, would be needed to test the n-3 PUFAs as a possible therapy. The trials performed to date have given very mixed results (Table 1.2). The results of the present work may help to explain why the past clinical studies have been inconclusive.

Of the 5 clinical trials that have been published (Table 1.2), only 2 found significant improvement in seizure control (Schlanger et al., 2002; Al Khayat et al., 2010). The other studies reported transient or negative results.

Significantly, the 2 studies that found improvements in seizure control were the two studies that had a longer duration of treatment ( 6 months). The 3 negative studies made use of a shorter 3 month trial period. It appears that a long period of exposure is required in humans to raise seizure threshold.

This would be in agreement with our animal studies, in combination with the known turnover rate of DHA in humans. In our animal studies, increases in ADT were only seen following 3 months of supplementation, which was also reported by Gilby et al. (2009). A priori, this might suggest that seizure protection should be seen in humans after 3 months. As noted above, however, the half life of fatty acids in adipose tissue is longer in humans compared to rats (Pihl et al., 1950; Hirsch et al., 1960). Thus, it might be expected that a clinical exposure of up to 6 months might be necessary to produce significant improvement.

Further to clinical studies, the studies that produced significant anti-seizures effects used higher doses as well as longer periods of exposure. Schlanger and colleagues, for instance, administered approximately $3.25 \mathrm{~g}$ of n-3 PUFAs per day to his patients, as compared to approximately $1-2 \mathrm{~g}$ per day in the negative studies. (Al Khayat et al. administered about a gram a day, but their subjects were young children.) Moreover, both Schlanger and Al Khayat 
administered more DHA than EPA, whereas the other studies gave their patients more EPA than DHA. DHA is readily incorporated into the brain, whereas EPA is almost all betaoxidized after entry into the brain (Chen et al., 2009). Although both molecules have anticonvulsant properties in vitro, it is unlikely that EPA has anticonvulsant properties in vivo due to its beta oxidation in the brain (although some EPA may be converted to DHA in the periphery). Therefore, clinical trials should use DHA, and should use a higher dose of 4-6g. Doses this high should not be toxic, since they would be within the limits of consumption in populations which consume a lot of seafood (Nobmann et al., 2005; Johnson et al., 2009).

n-3 PUFAs could also have clinical relevance in the treatment of status epilepticus. Chapter 7 demonstrated that n-3 PUFAs have anticonvulsant effects when administered i.v. During status epilepticus, drugs are delivered i.v. in order to stop the seizures. If a first drug fails, a second drug is tried. If the second drug fails, more drugs are tried until anesthesia is induced (Shorvon, 2011). n-3 PUFAs could potentially be used prior to induction of anesthesia in an attempt to suppress the seizures.

\subsection{PUFA and the Ketogenic Diet}

Is it possible that the anticonvulsant effects of the n-3 PUFAs contribute to the effects of the ketogenic diet?

The ketogenic diet was developed in the 1920s and is still used today to treat intractable seizures in children. The mechanism of action of this diet has yet to be solved, although many 
possible mechanisms of action have been suggested (Cunnane et al., 2002; Hartman et al., 2007).

One possible mechanism of action that has been suggested involves PUFAs (Cunnane et al., 2002). Children on the ketogenic diet have been reported to have increased circulating blood levels of the PUFAs. DHA, EPA, ALA, and AA have all been reported to be elevated $50-400 \%$ in both the phospholipid and unesterified pools of children on the diet (Fraser et al., 2003; Dahlin et al., 2007). In animals, the KD has also been shown to have a $15 \%$ increase in n-3 PUFAs in the brain (Taha et al., 2005).

Further, it is known that n-3 PUFAs modulate potassium conductances (Xu et al., 2008). Cerebrospinal fluid (CFS) taken from children on the KD has also been shown to increase potassium conductances when applied to Shaker K channel transfected oocytes, whereas CFS from the same children pre-KD had had no effect (Xu et al., 2008). This channel was also modulated by the application of n-3 PUFAs, but not by the application of the ketone bodies. $\mathrm{Xu}$ et al. therefore suggest that the effect of the CFS was due to PUFA modulation of the Shaker K channel (Xu et al., 2008).

It is therefore possible that the ketogenic diet works through elevating n-3 PUFAs in plasma and ultimately raising n-3 PUFAs in the brain. A correlate of this is that it might be useful to incorporate more PUFAs into the ketogenic diet. The ketogenic diet has classically been based on saturated fats (Hartman et al., 2007). Switching to unsaturated fats in the diet might possibly raise the levels of n-3 PUFAs in the brain even more, and possibly produce stronger anticonvulsant effects. Only one animal study has looked at a flaxseed based KD diet 
in the PTZ infusion model. The flaxseed KD provided the highest percentage of protected animals, although this difference was not statistically significant (Likhodii et al., 2000).

One question that needs to be discussed related to PUFAs and the ketogenic diet correlates to onset of anticonvulsant effects. The ketogenic diet is effective within days (Freeman et al., 2007), whereas PUFA supplementation takes months to raise thresholds. Even fasting in preparation for the ketogenic diet raises thresholds. Why is there such a difference in latency between the two treatments?

The answer probably relates to how quickly levels of unesterified PUFAs rise in the

blood. Fasting rapidly increases the unesterified lipid pool (Reichl, 1972). The unesterified fatty acid pool is the lipid pool available to the brain (Hamilton et al., 2001). It is possible that fasting could cause a significant increase in unesterified DHA in blood to increase brain DHA, which could convey anticonvulsant properties. The time course of the effects of PUFA supplementation on unesterified PUFAs in the blood has not yet been measured. It is hypothesized, however, that unesterified PUFA levels take months to rise with dietary supplementation, due to the incorporation of PUFAs into chylomicrons. (An experiment to test this hypothesis is proposed in the Future Experiment section. See below).

\subsection{Future Studies}

The present thesis has shown that n-3 PUFAs, and more specifically DHA, have anticonvulsant effects in vivo when given chronically, sub-chronically and acutely. It leaves a 
number of unresolved questions, however. The following section will further discuss the future experiments that might be completed to answer these questions.

\section{1) Measuring DHA profile in blood and brain during chronic n-3 PUFA supplementation}

Experiment 3 demonstrated that the effects of chronic fish oil administration take a long time to appear. This is in agreement with our own previous work and with the work of Gilby et al. (2009).

This long latency to effect is odd, as we also have found that acute administration of n3 PUFAs raises thresholds within minutes. We hypothesize that acute administration raises unesterified DHA levels rapidly, whereas dietary supplementation takes months to raise unesterified DHA levels.

To test this hypothesis, we suggest repeating the chronic fish oil supplementation study with regular measurements of unesterified serum PUFA levels. Along with the implanted animals used for ADT measurements, sham animals on the diet will be sacrificed at regular intervals (monthly) to collect blood and brain samples. Using these samples, the DHA profile in serum and brain will be measured each month. We hypothesize that anticonvulsant effects (elevations in ADT) will correlate with an increase in unesterified serum DHA, which in turn will be associated with an increase in unesterified brain DHA.

\section{2) DHA incorporation in the brain following s.c. injection}

Experiment 5 confirmed the existence of an inverted $U$ dose-response relationship when DHA is administered s.c. Contrary to our hypothesis, however, the serum unesterified 
DHA concentrations were similar in animals that received the lower (effective) dose and in animals that received the higher (ineffective) dose.

We hypothesize that this paradox occurs because - at the higher dose - DHA is coming out of solution and forming micelles. The DHA in micelles would still appear as unesterified DHA in out serum assays, but would be unavailable to the brain.

We propose to test this hypothesis but testing blood and brain levels of unesterified DHA at effective and (higher) ineffective s.c. doses. Due to the small concentrations of unesterified DHA in the brain, we propose to use radiolabelled DHA in this experiment since radiolabelled DHA can be measured even if concentrations are low. We hypothesize that the effective dose will produce more radioactivity in brain assays than the higher, ineffective dose.

\section{3) Effect of duration of DHA infusion on seizure protection}

In Experiment 7, we tested the effects of dose and time after infusion on the anticonvulsant effects of i.v. infusions of DHA. We did not, however, test the effects of the duration of the infusion on the anticonvulsant effects of DHA, since all subjects were infused for 5 minutes. Voskuyl and colleagues (1998) infused for a period of 30 minutes and reported more dramatic effects than the effects we observed (Voskuyl et al., 1998). It is possible that stronger effects would be seen if longer durations of infusion were used.

We suggest a "duration of infusion - response" study to analyse the effects of varying the periods of infusion on anticonvulsant response. 


\section{4) Direct vs. Systemic effect of DHA on seizure protection}

In all of the experiments in this thesis, DHA has been administered systemically through either p.o., s.c., i.p., or i.v. administration. We have assumed that DHA works by affecting function in the brain. This has never actually been demonstrated, however, and experiments with systemic administration of DHA cannot rule out the possibility that DHA conveys its anticonvulsant effects through some sort of peripheral modulation that indirectly affects the brain.

To eliminate the possibility that DHA conveys its effects through systemic modulation, we propose that the anticonvulsant effects of DHA should be tested after intracerebroventricular infusion.

\section{5) Modulation of sodium and calcium currents in amygdala and cortex}

This research doesn't propose any mechanism of action. From previous experiment in cardiomyocytes, n-3 PUFAs affect sodium and calcium currents (Kang and Leaf, 1994; Kang et al., 1995; Xiao et al., 1995; Xiao et al., 1998). In the hippocampus, application of DHA and EPA decreased sodium and calcium currents, with a reduction in action potential frequency (Vreugdenhil et al., 1996; Xiao and Li, 1999). Our work has focused on afterdischarge threshold in both the amygdala and cortex.

We therefore plan to perform electrophysiological studies in order to evaluate the effect of n-3 PUFAs on sodium currents and action potentials in cortical and amygdala slices, regions where we found an increase in ADT following n-3 PUFA supplementation. 


\section{6) Direct binding of n-3 PUFAs to the VDSC}

The experiments in the present thesis have been behavioural rather than mechanistic. Previous work, however suggests the hypothesis that DHA works by modulation of the VDSC in the brain. Previous work in cardiomyocytes further suggests that unesterified n-3 PUFAs bind to the VDSC, whereas esterified n-3 PUFAs do not (Kang and Leaf, 1996).

Future work might verify that n-3 PUFAs, and more specifically DHA, bind to the VDSC in brain tissue. Using brain homogenates and radiolabelled phenytoin, a known antagonist of the VDSC in brain tissue (Francis and Burnham, 1992), competition assays using various concentrations of "cold" n-3 PUFAs - could be used to correlate VDSC binding affinities to anticonvulsant effects as measured in vivo.

\section{7) Evaluation of the anticonvulsant effects of DHA in other seizure models}

The present research made use of only 2 seizure models, the maximal PTZ model and the electrical afterdischarge threshold model. These were used in order to remain consistent with our group's and the field's previous work.

The drug industry, however, employs at least 3 seizure models to screen for anticonvulsant potency. These 3 are the: the MES model, the amygdala kindling model, and the s.c. PTZ model. The MES model is the typical test used to screen for molecules which work against tonic-clonic seizures, whereas the amygdala model is a model of temporal lobe epilepsy - the hardest type of epilepsy to treat - and the s.c. PTZ model is a model of absence seizures (Fisher, 1989). 
Future work could look at the anticonvulsant effects of DHA in these 3 models. This would provide information on which human seizure types n-3 PUFAs might be effective against.

\section{8) Additive effects of traditional anticonvulsants with DHA}

Animal studies have found that administration of sub-therapeutic doses of n-3 PUFAs along with sub-therapeutic doses of antidepressants combined to have additive effects and decreased the latency to onset of action (Carlezon et al., 2005; Venna et al., 2009)

Clinically, epileptic patients would take n-3 PUFAs orally along with their AEDs. The present thesis has demonstrated that chronic oral administration of n-3 PUFAs resulted in an increase in seizure threshold compared to the control diet. It would be interesting, however, to test whether chronic n-3 PUFA administration along with AED medication combine to have an additive effect. Moreover, it would be interesting to see whether the latency of onset to anticonvulsant actions is shortened with the addition of AEDs.

\section{9) Replication of Bruzzese}

The present thesis attempted to replicate Bruzzese with some minor modification to the experiment. More specifically, we used a different strain of rats and did not make use of tocopherol, an anti-oxidant, to stay consistent with the remainder of our work.

Both groups found anticonvulsant effects, however, Bruzzese found dramatic effects whereas our results were more subtle. Future work should attempt to exactly replicate Bruzzese in order to see whether the dramatic effects seen by Bruzzese can be achieved. 


\subsection{Conclusion}

The present research suggests that n-3 PUFAs, and DHA more specifically, have anticonvulsant properties in vivo. n-3 PUFAs were found to be anticonvulsant in the 2 seizure models used in this research, the electrical threshold model and the maximal PTZ model. Moreover, n-3 PUFAs were anticonvulsant when administered chronically, sub-chronically and acutely.

These anticonvulsant effects may be mediated through an increase of the unesterified DHA pool in serum, which in turn increases brain unesterified DHA. Increased brain unesterified DHA could increase modulation of the VDSC, stabilizing the channel in its in inactive phase and retarding AP firing.

More research needs to be conducted in order to confirm the anticonvulsant properties of DHA in other seizure models. In vitro studies also need to be performed to determine a mechanism of action for the anticonvulsant properties of DHA.

Although it is too early to make any conclusive statement, the present research presents promising data suggesting that one day n-3 PUFAs could potentially be used clinically in the treatment of epilepsy. 


\section{REFERENCES}

Aicardi J (1988) Epileptic syndromes in childhood. Epilepsia 29 Suppl 3:S1-5.

Al Khayat HA, Awadalla MM, Al Wakad A, Marzook ZA (2010) Polyunsaturated fatty acids in children with idiopathic intractable epilepsy: Serum levels and therapeutic response. J Pediatr Neurol 8:175185.

Albright PS (1983) Effects of carbamazepine, clonazepam, and phenytoin on seizure threshold in amygdala and cortex. Exp Neurol 79:11-17.

Astorg P, Arnault N, Czernichow S, Noisette N, Galan P, Hercberg S (2004) Dietary intakes and food sources of n-6 and n-3 PUFA in French adult men and women. Lipids 39:527-535.

Balabanov PP, Zahariev ZI, Mateva NG (2008) Evaluation of the factors affecting the quality of life and total costs in epilepsy patients on monotherapy with carbamazepine and valproate. Folia Med (Plovdiv) 50:18-23.

Banerjee PN, Hauser WA (2008) Incidence and prevalence. In: Epilepsy: A comprehensive textbook, 2nd Edition (Engle J, Pedley TA, eds). Philadelphia: Lippincott Williams \& Wilkins.

Bang HO, Dyerberg J, Nielsen AB (1971) Plasma lipid and lipoprotein pattern in Greenlandic Westcoast Eskimos. Lancet 1:1143-1145.

Bang HO, Dyerberg J, Sinclair HM (1980) The composition of the Eskimo food in north western Greenland. Am J Clin Nutr 33:2657-2661.

Barcelo-Coblijn G, Kitajka K, Puskas LG, Hogyes E, Zvara A, Hackler L, Jr., Farkas T (2003) Gene expression and molecular composition of phospholipids in rat brain in relation to dietary $n-6$ to $n-3$ fatty acid ratio. Biochim Biophys Acta 1632:72-79.

Barnes J, Scahill RI, Schott JM, Frost C, Rossor MN, Fox NC (2005) Does Alzheimer's disease affect hippocampal asymmetry? Evidence from a cross-sectional and longitudinal volumetric MRI study. Dement Geriatr Cogn Disord 19:338-344.

Bazan NG, Aveldano de Caldironi MI, Rodriguez de Turco EB (1981) Rapid release of free arachidonic acid in the central nervous system due to stimulation. Prog Lipid Res 20:523-529.

Bazan NG, Jr. (1970) Effects of ischemia and electroconvulsive shock on free fatty acid pool in the brain. Biochim Biophys Acta 218:1-10.

Bazan NG, Jr. (1971) Changes in free fatty acids of brain by drug-induced convulsions, electroshock and anaesthesia. J Neurochem 18:1379-1385.

Belayev L, Marcheselli VL, Khoutorova L, Rodriguez de Turco EB, Busto R, Ginsberg MD, Bazan NG (2005) Docosahexaenoic acid complexed to albumin elicits high-grade ischemic neuroprotection. Stroke 36:118-123. 
Belayev L, Khoutorova L, Atkins KD, Bazan NG (2009) Robust docosahexaenoic acid-mediated neuroprotection in a rat model of transient, focal cerebral ischemia. Stroke 40:3121-3126.

Ben-Menachem E, Manon-Espaillat R, Ristanovic R, Wilder BJ, Stefan H, Mirza W, Tarver WB, Wernicke JF (1994) Vagus nerve stimulation for treatment of partial seizures: 1 . A controlled study of effect on seizures. First International Vagus Nerve Stimulation Study Group. Epilepsia 35:616-626.

Ben-Menachem E (2002) Vagus-nerve stimulation for the treatment of epilepsy. Lancet Neurol 1:477482.

Billman GE, Hallaq H, Leaf A (1994) Prevention of ischemia-induced ventricular fibrillation by omega 3 fatty acids. Proc Natl Acad Sci U S A 91:4427-4430.

Borowicz KK, Luszczki JJ, Duda AM, Czuczwar SJ (2003) Effect of topiramate on the anticonvulsant activity of conventional antiepileptic drugs in two models of experimental epilepsy. Epilepsia 44:640646.

Bough KJ, Chen RS, Eagles DA (1999) Path analysis shows that increasing ketogenic ratio, but not betahydroxybutyrate, elevates seizure threshold in the Rat. Dev Neurosci 21:400-406.

Bousquet M, Gibrat C, Saint-Pierre M, Julien C, Calon F, Cicchetti F (2009) Modulation of brain-derived neurotrophic factor as a potential neuroprotective mechanism of action of omega-3 fatty acids in a parkinsonian animal model. Prog Neuropsychopharmacol Biol Psychiatry 33:1401-1408.

Bragdon JH, Gordon RS, Jr. (1958) Tissue distribution of C14 after the intravenous injection of labeled chylomicrons and unesterified fatty acids in the rat. J Clin Invest 37:574-578.

Brenna JT, Salem N, Jr., Sinclair AJ, Cunnane SC (2009) alpha-Linolenic acid supplementation and conversion to $n-3$ long-chain polyunsaturated fatty acids in humans. Prostaglandins Leukot Essent Fatty Acids 80:85-91.

Bromfield E, Dworetzky B, Hurwitz S, Eluri Z, Lane L, Replansky S, Mostofsky D (2008) A randomized trial of polyunsaturated fatty acids for refractory epilepsy. Epilepsy Behav 12:187-190.

Bruzzese T (2005) Use of highly concentrated compositions of selected n-3 fatty acids for the treatment of central nervous system disturbances. 2005/070411A1

Burdge GC, Jones AE, Wootton SA (2002) Eicosapentaenoic and docosapentaenoic acids are the principal products of alpha-linolenic acid metabolism in young men*. Br J Nutr 88:355-363.

Burdge GC, Wootton SA (2002) Conversion of alpha-linolenic acid to eicosapentaenoic, docosapentaenoic and docosahexaenoic acids in young women. Br J Nutr 88:411-420.

Burdge GC, Finnegan YE, Minihane AM, Williams CM, Wootton SA (2003) Effect of altered dietary n-3 fatty acid intake upon plasma lipid fatty acid composition, conversion of [13C]alpha-linolenic acid to longer-chain fatty acids and partitioning towards beta-oxidation in older men. Br J Nutr 90:311-321. 
Burnham WM (2007) Antiseizure drugs In: Principles of Medical Pharmacology, 7th Edition (Kalant $\mathrm{H}$, Grant, D.M. and Mitchell, J., ed), pp 223-235. Toronto: Saunders.

Calder PC (2009) Polyunsaturated fatty acids and inflammatory processes: New twists in an old tale. Biochimie 91:791-795.

Calon F, Lim GP, Yang F, Morihara T, Teter B, Ubeda O, Rostaing P, Triller A, Salem N, Jr., Ashe KH, Frautschy SA, Cole GM (2004) Docosahexaenoic acid protects from dendritic pathology in an Alzheimer's disease mouse model. Neuron 43:633-645.

Camfield P, Camfield C (2010) Idiopathic generalized epilepsy with generalized tonic-clonic seizures (IGE-GTC): a population-based cohort with >20 year follow up for medical and social outcome. Epilepsy Behav 18:61-63.

Carlezon WA, Jr., Mague SD, Parow AM, Stoll AL, Cohen BM, Renshaw PF (2005) Antidepressant-like effects of uridine and omega-3 fatty acids are potentiated by combined treatment in rats. Biol Psychiatry 57:343-350.

Carrie I, Clement M, de Javel D, Frances H, Bourre JM (2000) Phospholipid supplementation reverses behavioral and biochemical alterations induced by $n-3$ polyunsaturated fatty acid deficiency in mice. J Lipid Res 41:473-480.

Catterall WA, Kalume F, Oakley JC (2010) NaV1.1 channels and epilepsy. J Physiol 588:1849-1859.

Chen CT, Green JT, Orr SK, Bazinet RP (2008a) Regulation of brain polyunsaturated fatty acid uptake and turnover. Prostaglandins Leukot Essent Fatty Acids 79:85-91.

Chen CT, Ma DW, Kim JH, Mount HT, Bazinet RP (2008b) The low density lipoprotein receptor is not necessary for maintaining mouse brain polyunsaturated fatty acid concentrations. J Lipid Res 49:147152.

Chen CT, Liu Z, Ouellet M, Calon F, Bazinet RP (2009) Rapid beta-oxidation of eicosapentaenoic acid in mouse brain: an in situ study. Prostaglandins Leukot Essent Fatty Acids 80:157-163.

Chong DJ, Bazil CW (2010) Update on anticonvulsant drugs. Curr Neurol Neurosci Rep 10:308-318.

Chung WL, Chen JJ, Su HM (2008) Fish oil supplementation of control and (n-3) fatty acid-deficient male rats enhances reference and working memory performance and increases brain regional docosahexaenoic acid levels. J Nutr 138:1165-1171.

Clark KB, Naritoku DK, Smith DC, Browning RA, Jensen RA (1999) Enhanced recognition memory following vagus nerve stimulation in human subjects. Nat Neurosci 2:94-98.

Climax J, Sewell RD (1981) Modification of convulsive behaviour and body temperature in mice by intracerebroventricular administration of prostaglandins, arachidonic acid and the soluble acetylsalicylic acid salt lysine acetylsalicylate. Arch Int Pharmacodyn Ther 250:254-265. 
Conklin SM, Gianaros PJ, Brown SM, Yao JK, Hariri AR, Manuck SB, Muldoon MF (2007) Long-chain omega-3 fatty acid intake is associated positively with corticolimbic gray matter volume in healthy adults. Neurosci Lett 421:209-212.

Cunnane SC, Anderson MJ (1997) The majority of dietary linoleate in growing rats is beta-oxidized or stored in visceral fat. J Nutr 127:146-152.

Cunnane SC, Musa K, Ryan MA, Whiting S, Fraser DD (2002) Potential role of polyunsaturates in seizure protection achieved with the ketogenic diet. Prostaglandins Leukot Essent Fatty Acids 67:131135.

Dahlin M, Hjelte L, Nilsson S, Amark P (2007) Plasma phospholipid fatty acids are influenced by a ketogenic diet enriched with n-3 fatty acids in children with epilepsy. Epilepsy Res 73:199-207.

DeGiorgio CM, Miller P, Meymandi S, Gornbein JA (2008) n-3 fatty acids (fish oil) for epilepsy, cardiac risk factors, and risk of SUDEP: clues from a pilot, double-blind, exploratory study. Epilepsy Behav 13:681-684.

DeMar JC, Jr., Ma K, Bell JM, Rapoport SI (2004) Half-lives of docosahexaenoic acid in rat brain phospholipids are prolonged by 15 weeks of nutritional deprivation of $n-3$ polyunsaturated fatty acids. J Neurochem 91:1125-1137.

Demar JC, Jr., Ma K, Chang L, Bell JM, Rapoport SI (2005) alpha-Linolenic acid does not contribute appreciably to docosahexaenoic acid within brain phospholipids of adult rats fed a diet enriched in docosahexaenoic acid. J Neurochem 94:1063-1076.

DeMar JC, Jr., Lee HJ, Ma K, Chang L, Bell JM, Rapoport SI, Bazinet RP (2006a) Brain elongation of linoleic acid is a negligible source of the arachidonate in brain phospholipids of adult rats. Biochim Biophys Acta 1761:1050-1059.

DeMar JC, Jr., Ma K, Bell JM, Igarashi M, Greenstein D, Rapoport SI (2006b) One generation of n-3 polyunsaturated fatty acid deprivation increases depression and aggression test scores in rats. J Lipid Res 47:172-180.

Denomme J, Stark KD, Holub BJ (2005) Directly quantitated dietary (n-3) fatty acid intakes of pregnant Canadian women are lower than current dietary recommendations. J Nutr 135:206-211.

Devinsky O, Barr WB, Vickrey BG, Berg AT, Bazil CW, Pacia SV, Langfitt JT, Walczak TS, Sperling MR, Shinnar S, Spencer SS (2005) Changes in depression and anxiety after resective surgery for epilepsy. Neurology 65:1744-1749.

Diamond DM, Bennett MC, Fleshner M, Rose GM (1992) Inverted-U relationship between the level of peripheral corticosterone and the magnitude of hippocampal primed burst potentiation. Hippocampus 2:421-430.

Duncan JS (2007) Epilepsy surgery. Clin Med 7:137-142. 
Dyerberg J, Bang HO, Hjorne N (1975) Fatty acid composition of the plasma lipids in Greenland Eskimos. Am J Clin Nutr 28:958-966.

Ebert U, Cramer S, Loscher W (1997) Phenytoin's effect on the spread of seizure activity in the amygdala kindling model. Naunyn Schmiedebergs Arch Pharmacol 356:341-347.

Edmond J (2001) Essential polyunsaturated fatty acids and the barrier to the brain: the components of a model for transport. J Mol Neurosci 16:181-193; discussion 215-121.

Edwards R, Peet M, Shay J, Horrobin D (1998) Omega-3 polyunsaturated fatty acid levels in the diet and in red blood cell membranes of depressed patients. J Affect Disord 48:149-155.

Elger G, Hoppe C, Falkai P, Rush AJ, Elger CE (2000) Vagus nerve stimulation is associated with mood improvements in epilepsy patients. Epilepsy Res 42:203-210.

Engel J, Williamson PD (2008) Limbic Seizures. In: Epilepsy: A Comprehensive Textbook, second Edition (Engel J, Pedley TA, eds). Philadelphia: Lippincott Williams \& Wilkins.

Engel J, Jr. (1998) Classifications of the International League Against Epilepsy: time for reappraisal. Epilepsia 39:1014-1017.

Engel J, Jr., Williamson PD, Berg AT, Wolf P (2008) Classification of epileptic seizures. In: Epilepsy: A comprehensive textbook, 2nd Edition (Engel J, Jr., Pedley TA, eds). Philadelphia: Lippincott, Williams \& Wilkins.

Faught E, Glauser TA (2008) Topiramate. In: Epilepsy: A Comprehensive Textbook, 2nd Edition (Engel J, Pedley TA, eds). Philadelphia.

Fedorova I, Salem N, Jr. (2006) Omega-3 fatty acids and rodent behavior. Prostaglandins Leukot Essent Fatty Acids 75:271-289.

Ferraz AC, Delattre AM, Almendra RG, Sonagli M, Borges C, Araujo P, Andersen ML, Tufik S, Lima MM (2010) Chronic omega-3 fatty acids supplementation promotes beneficial effects on anxiety, cognitive and depressive-like behaviors in rats subjected to a restraint stress protocol. Behav Brain Res.

Fisher RS (1989) Animal models of the epilepsies. Brain Res Brain Res Rev 14:245-278.

Flood VM, Webb KL, Rochtchina E, Kelly B, Mitchell P (2007) Fatty acid intakes and food sources in a population of older Australians. Asia Pac J Clin Nutr 16:322-330.

Folch J, Lees M, Sloane Stanley GH (1957) A simple method for the isolation and purification of total lipides from animal tissues. J Biol Chem 226:497-509.

Frances H, Monier C, Bourre JM (1995) Effects of dietary alpha-linolenic acid deficiency on neuromuscular and cognitive functions in mice. Life Sci 57:1935-1947. 
Francis J, Burnham WM (1992) [3H]Phenytoin identifies a novel anticonvulsant-binding domain on voltage-dependent sodium channels. Mol Pharmacol 42:1097-1103.

Fraser DD, Hoehn K, Weiss S, MacVicar BA (1993) Arachidonic acid inhibits sodium currents and synaptic transmission in cultured striatal neurons. Neuron 11:633-644.

Fraser DD, Whiting S, Andrew RD, Macdonald EA, Musa-Veloso K, Cunnane SC (2003) Elevated polyunsaturated fatty acids in blood serum obtained from children on the ketogenic diet. Neurology 60:1026-1029.

Fredrickson DS, Gordon RS, Jr. (1958) Transport of fatty acids. Physiol Rev 38:585-630.

Freeman JM, Kossoff EH (2010) Ketosis and the ketogenic diet, 2010: advances in treating epilepsy and other disorders. Adv Pediatr 57:315-329.

Freeman MP, Hibbeln JR, Wisner KI, Davis JM, Mischoulon D, Peet M, Keck PE, Marangell LB, Richardson AJ, Lake J, Stoll AL (2006) Omega-3 fatty acids: evidence basis for treatment and future research in psychiatry. J Clin Psychiatry 68:1954 - 1967.

Furth SL, Casey JC, Pyzik PL, Neu AM, Docimo SG, Vining EP, Freeman JM, Fivush BA (2000) Risk factors for urolithiasis in children on the ketogenic diet. Pediatr Nephrol 15:125-128.

Galli C, Trzeciak HI, Paoletti R (1971) Effects of dietary fatty acids on the fatty acid composition of brain ethanolamine phosphoglyceride: Reciprocal replacement of $n-6$ and $n-3$ polyunsaturated fatty acids. Biochimica et Biophysica Acta (BBA) - Lipids and Lipid Metabolism 248:449-454.

Gao F, Kiesewetter D, Chang L, Ma K, Bell JM, Rapoport SI, Igarashi M (2009) Whole-body synthesissecretion rates of long-chain n-3 PUFAs from circulating unesterified alpha-linolenic acid in unanesthetized rats. J Lipid Res 50:749-758.

Gao F, Kiesewetter D, Chang L, Rapoport SI, Igarashi M (2010) Quantifying conversion of linoleic to arachidonic and other n-6 polyunsaturated fatty acids in unanesthetized rats. J Lipid Res 51:29402946.

Garfinkel AS, Baker N, Schotz MC (1967) Relationship of lipoprotein lipase activity to triglyceride uptake in adipose tissue. J Lipid Res 8:274-280.

Gastaut H, Gastaut JL, Goncalves e Silva GE, Fernandez Sanchez GR (1975) Relative frequency of different types of epilepsy: a study employing the classification of the International League Against Epilepsy. Epilepsia 16:457-461.

Gilby KL, Jans J, McIntyre DC (2009) Chronic omega-3 supplementation in seizure-prone versus seizure-resistant rat strains: a cautionary tale. Neuroscience 163:750-758.

Guberman A, Bruni J (1999) Essentials of Clinical Epilepsy, Second Edition. Boston: ButterworthHeinemann. 
Guimaraes J, Ribeiro JA (2010) Pharmacology of antiepileptic drugs in clinical practice. Neurologist 16:353-357.

Hahn A, Neubauer BA (2009) Sodium and potassium channel dysfunctions in rare and common idiopathic epilepsy syndromes. Brain Dev 31:515-520.

Hamilton JA, Johnson RA, Corkey B, Kamp F (2001) Fatty acid transport: the diffusion mechanism in model and biological membranes. J Mol Neurosci 16:99-108; discussion 151-107.

Handforth A, DeGiorgio CM, Schachter SC, Uthman BM, Naritoku DK, Tecoma ES, Henry TR, Collins SD, Vaughn BV, Gilmartin RC, Labar DR, Morris GL, 3rd, Salinsky MC, Osorio I, Ristanovic RK, Labiner DM, Jones JC, Murphy JV, Ney GC, Wheless JW (1998) Vagus nerve stimulation therapy for partial-onset seizures: a randomized active-control trial. Neurology 51:48-55.

Harkin LA, Bowser DN, Dibbens LM, Singh R, Phillips F, Wallace RH, Richards MC, Williams DA, Mulley JC, Berkovic SF, Scheffer IE, Petrou S (2002) Truncation of the GABA(A)-receptor gamma2 subunit in a family with generalized epilepsy with febrile seizures plus. Am J Hum Genet 70:530-536.

Harris WS, Mozaffarian D, Lefevre M, Toner CD, Colombo J, Cunnane SC, Holden JM, Klurfeld DM, Morris MC, Whelan J (2009) Towards establishing dietary reference intakes for eicosapentaenoic and docosahexaenoic acids. J Nutr 139:804S-819S.

Hartman AL, Gasior M, Vining EP, Rogawski MA (2007) The neuropharmacology of the ketogenic diet. Pediatr Neurol 36:281-292.

Hashimoto M, Tanabe Y, Fujii Y, Kikuta T, Shibata H, Shido O (2005) Chronic administration of docosahexaenoic acid ameliorates the impairment of spatial cognition learning ability in amyloid betainfused rats. J Nutr 135:549-555.

He C, Qu X, Cui L, Wang J, Kang JX (2009) Improved spatial learning performance of fat-1 mice is associated with enhanced neurogenesis and neuritogenesis by docosahexaenoic acid. Proc Natl Acad Sci U S A 106:11370-11375.

Hirsch J, Farquhar JW, Ahrens EH, Jr., Peterson ML, Stoffel W (1960) Studies of adipose tissue in man. A microtechnic for sampling and analysis. Am J Clin Nutr 8:499-511.

Hong S, Gronert K, Devchand PR, Moussignac RL, Serhan CN (2003) Novel docosatrienes and 17Sresolvins generated from docosahexaenoic acid in murine brain, human blood, and glial cells. Autacoids in anti-inflammation. J Biol Chem 278:14677-14687.

Huang SY, Yang HT, Chiu CC, Pariante CM, Su KP (2008) Omega-3 fatty acids on the forced-swimming test. J Psychiatr Res 42:58-63.

Igarashi M, DeMar JC, Jr., Ma K, Chang L, Bell JM, Rapoport SI (2007) Docosahexaenoic acid synthesis from alpha-linolenic acid by rat brain is unaffected by dietary n-3 PUFA deprivation. $J$ Lipid Res 48:1150-1158. 
ILAE (1981) Proposal for revised clinical and electroencephalographic classification of epileptic seizures. From the Commission on Classification and Terminology of the International League Against Epilepsy. Epilepsia 22:489-501.

Jenkins D, Spector RG (1973) The actions of folate and phenytoin on the rat heart in vivo and in vitro. Biochem Pharmacol 22:1813-1816.

Jennum P, Gyllenborg J, Kjellberg J (2011) The social and economic consequences of epilepsy: A controlled national study. Epilepsia.

Jicha GA, Markesbery WR (2010) Omega-3 fatty acids: potential role in the management of early Alzheimer's disease. Clin Interv Aging 5:45-61.

Joensen P (1986) Prevalence, incidence, and classification of epilepsy in the Faroes. Acta Neurol Scand 74:150-155.

Johnson JS, Nobmann ED, Asay E, Lanier AP (2009) Dietary intake of Alaska Native people in two regions and implications for health: the Alaska Native Dietary and Subsistence Food Assessment Project. Int J Circumpolar Health 68:109-122.

Kang JX, Leaf A (1994) Effects of long-chain polyunsaturated fatty acids on the contraction of neonatal rat cardiac myocytes. Proc Natl Acad Sci U S A 91:9886-9890.

Kang JX, Xiao YF, Leaf A (1995) Free, long-chain, polyunsaturated fatty acids reduce membrane electrical excitability in neonatal rat cardiac myocytes. Proc Natl Acad Sci U S A 92:3997-4001.

Kang JX, Leaf A (1996) Evidence that free polyunsaturated fatty acids modify Na+ channels by directly binding to the channel proteins. Proc Natl Acad Sci U S A 93:3542-3546.

Karpe F, Bickerton AS, Hodson L, Fielding BA, Tan GD, Frayn KN (2007) Removal of triacylglycerols from chylomicrons and VLDL by capillary beds: the basis of lipoprotein remnant formation. Biochem Soc Trans 35:472-476.

Keene DL (2006) A systematic review of the use of the ketogenic diet in childhood epilepsy. Pediatr Neurol 35:1-5.

Kitajka K, Puskas LG, Zvara A, Hackler L, Jr., Barcelo-Coblijn G, Yeo YK, Farkas T (2002) The role of n-3 polyunsaturated fatty acids in brain: modulation of rat brain gene expression by dietary $n-3$ fatty acids. Proc Natl Acad Sci U S A 99:2619-2624.

Komprda T, Zelenka J, Fajmonova E, Fialova M, Kladroba D (2005) Arachidonic acid and long-chain n-3 polyunsaturated fatty acid contents in meat of selected poultry and fish species in relation to dietary fat sources. J Agric Food Chem 53:6804-6812.

Kossoff EH, Zupec-Kania BA, Rho JM (2009) Ketogenic diets: an update for child neurologists. J Child Neurol 24:979-988. 
Krahl SE, Clark KB, Smith DC, Browning RA (1998) Locus coeruleus lesions suppress the seizureattenuating effects of vagus nerve stimulation. Epilepsia 39:709-714.

Kris-Etherton PM, Grieger JA, Etherton TD (2009) Dietary reference intakes for DHA and EPA. Prostaglandins Leukot Essent Fatty Acids 81:99-104.

Krumholz A (2009) Driving issues in epilepsy: past, present, and future. Epilepsy Curr 9:31-35.

Lakhwani L, Tongia SK, Pal VS, Agrawal RP, Nyati P, Phadnis P (2007) Omega-3 fatty acids have antidepressant activity in forced swimming test in Wistar rats. Acta Pol Pharm 64:271-276.

Lefevre F, Aronson N (2000) Ketogenic diet for the treatment of refractory epilepsy in children: A systematic review of efficacy. Pediatrics 105:E46.

Levant B, Ozias MK, Davis PF, Winter M, Russell KL, Carlson SE, Reed GA, McCarson KE (2008) Decreased brain docosahexaenoic acid content produces neurobiological effects associated with depression: Interactions with reproductive status in female rats. Psychoneuroendocrinology 33:12791292.

Likhodii SS, Musa K, Mendonca A, Dell C, Burnham WM, Cunnane SC (2000) Dietary fat, ketosis, and seizure resistance in rats on the ketogenic diet. Epilepsia 41:1400-1410.

Likhodii SS, Burnham WM (2002) Ketogenic diet: does acetone stop seizures? Med Sci Monit 8:HY1924.

Likhodii SS, Musa K, Cunnane SC (2002) Breath acetone as a measure of systemic ketosis assessed in a rat model of the ketogenic diet. Clin Chem 48:115-120.

Likhodii SS, Serbanescu I, Cortez MA, Murphy P, Snead OC, 3rd, Burnham WM (2003) Anticonvulsant properties of acetone, a brain ketone elevated by the ketogenic diet. Ann Neurol 54:219-226.

Lim SY, Hoshiba J, Salem N, Jr. (2005) An extraordinary degree of structural specificity is required in neural phospholipids for optimal brain function: $n-6$ docosapentaenoic acid substitution for docosahexaenoic acid leads to a loss in spatial task performance. J Neurochem 95:848-857.

Lin PY, Huang SY, Su KP (2010) A meta-analytic review of polyunsaturated fatty acid compositions in patients with depression. Biol Psychiatry 68:140-147.

Lonsdale D, Burnham WM (2003) The anticonvulsant effects of progesterone and 5alphadihydroprogesterone on amygdala-kindled seizures in rats. Epilepsia 44:1494-1499.

Lucas M, Asselin G, Plourde M, Cunnane SC, Dewailly E, Dodin S (2010) n-3 Fatty acid intake from marine food products among Quebecers: comparison to worldwide recommendations. Public Health Nutr 13:63-70. 
Macdonald RL, Rogawski MA (2008) Cellular effects of antiepileptic drugs. In: Epilepsy: A comprehensive textbook, Second Edition (Engel J, Pedley TA, eds). Philadelphia: Lippincott Williams \& Wilkins.

Macdonald RL, Kang JQ, Gallagher MJ (2010) Mutations in GABAA receptor subunits associated with genetic epilepsies. J Physiol 588:1861-1869.

Mansbach CM, Siddiqi SA (2010) The biogenesis of chylomicrons. Annu Rev Physiol 72:315-333.

Marangell LB, Martinez JM, Zboyan HA, Kertz B, Kim HF, Puryear LJ (2003) A double-blind, placebocontrolled study of the omega-3 fatty acid docosahexaenoic acid in the treatment of major depression. Am J Psychiatry 160:996-998.

Marcheselli VL, Hong S, Lukiw WJ, Tian XH, Gronert K, Musto A, Hardy M, Gimenez JM, Chiang N, Serhan CN, Bazan NG (2003) Novel docosanoids inhibit brain ischemia-reperfusion-mediated leukocyte infiltration and pro-inflammatory gene expression. J Biol Chem 278:43807-43817.

Marini C, Harkin LA, Wallace RH, Mulley JC, Scheffer IE, Berkovic SF (2003) Childhood absence epilepsy and febrile seizures: a family with a GABA(A) receptor mutation. Brain 126:230-240.

Martins JG (2009) EPA but not DHA appears to be responsible for the efficacy of omega-3 long chain polyunsaturated fatty acid supplementation in depression: evidence from a meta-analysis of randomized controlled trials. J Am Coll Nutr 28:525-542.

Matthews H, Granger N, Wood J, Skelly B (2011) Effects of essential fatty acid supplementation in dogs with idiopathic epilepsy: A clinical trial. Vet J.

Mayes PA, Botham KM (2003) Lipid Transport \& Storage. In: Harper's Illustrated Biochemistry, 26th Edition (Murray RK, Granner DK, Mayes PA, Rodwell VW, eds). New York: McGraw-Hill.

McNamara JO, Rigsbee LC, Butler LS, Shin C (1989) Intravenous phenytoin is an effective anticonvulsant in the kindling model. Ann Neurol 26:675-678.

Mead JF (1963) LIPID METABOLISM. Annu Rev Biochem 32:241-268.

Meisler MH, Kearney JA (2005) Sodium channel mutations in epilepsy and other neurological disorders. J Clin Invest 115:2010-2017.

Meldrum BS (2008) Molecular targets for novel antiepileptic drugs. In: Epilepsy: A comprehensive textbook, Second Edition (Engel J, Pedley TA, eds). Philadelphia: Lippincott Williams \& Wilkins.

Meyer BJ, Mann NJ, Lewis JL, Milligan GC, Sinclair AJ, Howe PR (2003) Dietary intakes and food sources of omega- 6 and omega-3 polyunsaturated fatty acids. Lipids 38:391-398.

Moriguchi T, Greiner RS, Salem N, Jr. (2000) Behavioral deficits associated with dietary induction of decreased brain docosahexaenoic acid concentration. J Neurochem 75:2563-2573. 
Moriguchi T, Salem N, Jr. (2003) Recovery of brain docosahexaenoate leads to recovery of spatial task performance. J Neurochem 87:297-309.

Morris GL, 3rd, Mueller WM (1999) Long-term treatment with vagus nerve stimulation in patients with refractory epilepsy. The Vagus Nerve Stimulation Study Group E01-E05. Neurology 53:1731-1735.

Morris R (1984) Developments of a water-maze procedure for studying spatial learning in the rat. J Neurosci Methods 11:47-60.

Moshé SL, Pedley TA (2008) The history and physical examination. In: Epilepsy: A Comprehensive Look, Second Edition (Engel J, Pedley TA, eds). Philadelphia: Lippincott, Williams \& Wilkins.

Mucha RF, Pinel PJ (1977) Postseizure inhibition of kindled seizures. Exp Neurol 54:266-282.

Musto AE, Gjorstrup P, Bazan NG (2011) The omega-3 fatty acid-derived neuroprotectin D1 limits hippocampal hyperexcitability and seizure susceptibility in kindling epileptogenesis. Epilepsia.

Muthugovindan D, Hartman AL (2010) Pediatric epilepsy syndromes. Neurologist 16:223-237.

Neal EG, Chaffe H, Schwartz RH, Lawson MS, Edwards N, Fitzsimmons G, Whitney A, Cross JH (2008) The ketogenic diet for the treatment of childhood epilepsy: a randomised controlled trial. Lancet Neurol 7:500-506.

Neal EG, Chaffe H, Schwartz RH, Lawson MS, Edwards N, Fitzsimmons G, Whitney A, Cross JH (2009) A randomized trial of classical and medium-chain triglyceride ketogenic diets in the treatment of childhood epilepsy. Epilepsia 50:1109-1117.

Nemets B, Stahl Z, Belmaker RH (2002) Addition of omega-3 fatty acid to maintenance medication treatment for recurrent unipolar depressive disorder. Am J Psychiatry 159:477-479.

Neubauer BA, Waldegger S, Heinzinger J, Hahn A, Kurlemann G, Fiedler B, Eberhard F, Muhle H, Stephani U, Garkisch S, Eeg-Olofsson O, Muller U, Sander T (2008) KCNQ2 and KCNQ3 mutations contribute to different idiopathic epilepsy syndromes. Neurology 71:177-183.

Neuman RS (1986) Suppression of penicillin-induced focal epileptiform activity by locus ceruleus stimulation: mediation by an alpha 1-adrenoceptor. Epilepsia 27:359-366.

Nobmann ED, Ponce R, Mattil C, Devereux R, Dyke B, Ebbesson SO, Laston S, MacCluer J, Robbins D, Romenesko T, Ruotolo G, Wenger CR, Howard BV (2005) Dietary intakes vary with age among Eskimo adults of Northwest Alaska in the GOCADAN study, 2000-2003. J Nutr 135:856-862.

O'Sullivan TA, Ambrosini G, Beilin LJ, Mori TA, Oddy WH (2011) Dietary intake and food sources of fatty acids in Australian adolescents. Nutrition 27:153-159.

Oksman M, livonen H, Hogyes E, Amtul Z, Penke B, Leenders I, Broersen L, Lutjohann D, Hartmann T, Tanila $H$ (2006) Impact of different saturated fatty acid, polyunsaturated fatty acid and cholesterol containing diets on beta-amyloid accumulation in APP/PS1 transgenic mice. Neurobiol Dis 23:563-572. 
Ottman R, Winawer MR (2008) Genetic epidemiology. In: Epilepsy: A comprehensive textbook, 2nd Edition (Engle J, Pedley TA, eds). Philadelphia: Lippincott Williams \& Wilkins.

Ouellet M, Emond V, Chen CT, Julien C, Bourasset F, Oddo S, LaFerla F, Bazinet RP, Calon F (2009) Diffusion of docosahexaenoic and eicosapentaenoic acids through the blood-brain barrier: An in situ cerebral perfusion study. Neurochem Int 55:476-482.

Pages N, Maurois P, Delplanque B, Bac P, Martin JC, Du Q, Rapoport SI, Vamecq J (2011) Brain protection by rapeseed oil in magnesium-deficient mice. Prostaglandins Leukot Essent Fatty Acids 85:53-60.

Pawlosky R, Hibbeln J, Lin Y, Salem N, Jr. (2003) n-3 fatty acid metabolism in women. Br J Nutr 90:993994; discussion 994-995.

Peet M, Horrobin DF (2002) A dose-ranging study of the effects of ethyl-eicosapentaenoate in patients with ongoing depression despite apparently adequate treatment with standard drugs. Arch Gen Psychiatry 59:913-919.

Pellegrino LJ, Cushman AJ (1971) Use of Stereotaxic Technique. In: Methods in Psychobiology (Myers RD, ed). London: Academic Press inc.

Pellow S, Chopin P, File SE, Briley M (1985) Validation of open:closed arm entries in an elevated plusmaze as a measure of anxiety in the rat. J Neurosci Methods 14:149-167.

Penfield W, Jasper H (1954) Epilepsy and the Functional Anatomy of the Human Brain. Boston: Little, Brown and Company.

Pihl A, Block K, Anker HS (1950) The rates of synthesis of fatty acids and cholesterol in the adult rat studied with the aid of labeled acetic acid. J Biol Chem 183:10.

Pinel JP, Skelton R, Mucha RF (1976) Kindling-related changes in afterdischarge "thresholds". Epilepsia 17:197-206.

Plourde M, Cunnane SC (2007) Extremely limited synthesis of long chain polyunsaturates in adults: implications for their dietary essentiality and use as supplements. Appl Physiol Nutr Metab 32:619634.

Porsolt RD, Bertin A, Jalfre M (1977) Behavioral despair in mice: a primary screening test for antidepressants. Arch Int Pharmacodyn Ther 229:327-336.

Porta N, Bourgois B, Galabert C, Lecointe C, Cappy P, Bordet R, Vallee L, Auvin S (2009) Anticonvulsant effects of linolenic acid are unrelated to brain phospholipid cell membrane compositions. Epilepsia 50:65-71. 
Rabinovitz S, Mostofsky DI, Yehuda S (2004) Anticonvulsant efficiency, behavioral performance and cortisol levels: a comparison of carbamazepine (CBZ) and a fatty acid compound (SR-3).

Psychoneuroendocrinology 29:113-124.

Racine RJ (1972) Modification of seizure activity by electrical stimulation. II. Motor seizure. Electroencephalogr Clin Neurophysiol 32:281-294.

Ramani R (2008) Vagus nerve stimulation therapy for seizures. J Neurosurg Anesthesiol 20:29-35.

Ramsay RE, Uthman BM, Augustinsson LE, Upton AR, Naritoku D, Willis J, Treig T, Barolat G, Wernicke JF (1994) Vagus nerve stimulation for treatment of partial seizures: 2 . Safety, side effects, and tolerability. First International Vagus Nerve Stimulation Study Group. Epilepsia 35:627-636.

Rao JS, Ertley RN, DeMar JC, Jr., Rapoport SI, Bazinet RP, Lee HJ (2007a) Dietary n-3 PUFA deprivation alters expression of enzymes of the arachidonic and docosahexaenoic acid cascades in rat frontal cortex. Mol Psychiatry 12:151-157.

Rao JS, Ertley RN, Lee HJ, DeMar JC, Jr., Arnold JT, Rapoport SI, Bazinet RP (2007b) n-3 polyunsaturated fatty acid deprivation in rats decreases frontal cortex BDNF via a p38 MAPK-dependent mechanism. Mol Psychiatry 12:36-46.

Rapoport SI, Igarashi M, Gao F (2010) Quantitative contributions of diet and liver synthesis to docosahexaenoic acid homeostasis. Prostaglandins Leukot Essent Fatty Acids 82:273-276.

Reichl D (1972) Lipoprotein lipase activity in the adipose tissue of rats adapted to controlled feeding schedules. Biochem J 128:79-87.

Rho JM, Anderson GD, Donevan SD, White HS (2002) Acetoacetate, acetone, and dibenzylamine (a contaminant in I-(+)-beta-hydroxybutyrate) exhibit direct anticonvulsant actions in vivo. Epilepsia 43:358-361.

Robinson PJ, Noronha J, DeGeorge JJ, Freed LM, Nariai T, Rapoport SI (1992) A quantitative method for measuring regional in vivo fatty-acid incorporation into and turnover within brain phospholipids: review and critical analysis. Brain Res Brain Res Rev 17:187-214.

Rodbell M (1960) The removal and metabolism of chylomicrons by adipose tissue in vitro. J Biol Chem 235:1613-1620.

Rogawski MA, Loscher W (2004) The neurobiology of antiepileptic drugs. Nat Rev Neurosci 5:553-564.

Rogers PJ, Appleton KM, Kessler D, Peters TJ, Gunnell D, Hayward RC, Heatherley SV, Christian LM, McNaughton SA, Ness AR (2008) No effect of $n-3$ long-chain polyunsaturated fatty acid (EPA and DHA) supplementation on depressed mood and cognitive function: a randomised controlled trial. Br J Nutr 99:421-431.

Russo GL (2009) Dietary n-6 and n-3 polyunsaturated fatty acids: from biochemistry to clinical implications in cardiovascular prevention. Biochem Pharmacol 77:937-946. 
Salem N, Jr., Litman B, Kim HY, Gawrisch K (2001) Mechanisms of action of docosahexaenoic acid in the nervous system. Lipids 36:945-959.

Sanchez-Chapula J, Josephson IR (1983) Effect of phenytoin on the sodium current in isolated rat ventricular cells. J Mol Cell Cardiol 15:515-522.

Schlanger S, Shinitzky M, Yam D (2002) Diet enriched with omega-3 fatty acids alleviates convulsion symptoms in epilepsy patients. Epilepsia 43:103-104.

Schwab JM, Chiang N, Arita M, Serhan CN (2007) Resolvin E1 and protectin D1 activate inflammationresolution programmes. Nature 447:869-874.

Serhan CN, Clish CB, Brannon J, Colgan SP, Chiang N, Gronert K (2000) Novel functional sets of lipidderived mediators with antiinflammatory actions generated from omega-3 fatty acids via cyclooxygenase 2-nonsteroidal antiinflammatory drugs and transcellular processing. J Exp Med 192:1197-1204.

Serhan CN, Hong S, Gronert K, Colgan SP, Devchand PR, Mirick G, Moussignac RL (2002) Resolvins: a family of bioactive products of omega-3 fatty acid transformation circuits initiated by aspirin treatment that counter proinflammation signals. J Exp Med 196:1025-1037.

Serhan CN, Yacoubian S, Yang R (2008) Anti-inflammatory and proresolving lipid mediators. Annu Rev Pathol 3:279-312.

Serrano EE, Wilder BJ (1974) Intramuscular administration of diphenylhydantoin. Histologic follow-up studies. Arch Neurol 31:276-278.

Shafique S, Dalsing MC (2006) Vagus nerve stimulation therapy for treatment of drug-resistant epilepsy and depression. Perspect Vasc Surg Endovasc Ther 18:323-327.

Shorvon S (2011) The treatment of status epilepticus. Curr Opin Neurol 24:165-170.

Simopoulos AP (2000) Human requirement for N-3 polyunsaturated fatty acids. Poult Sci 79:961-970.

Sioen I, Devroe J, Inghels D, Terwecoren R, De Henauw S (2010) The influence of n-3 PUFA supplements and $n-3$ PUFA enriched foods on the n-3 LC PUFA intake of Flemish women. Lipids 45:313-320.

Sioen IA, Pynaert I, Matthys C, De Backer G, Van Camp J, De Henauw S (2006) Dietary intakes and food sources of fatty acids for Belgian women, focused on $n-6$ and $n-3$ polyunsaturated fatty acids. Lipids 41:415-422.

Song BJ, Elbert A, Rahman T, Orr SK, Chen CT, Febbraio M, Bazinet RP (2010) Genetic ablation of CD36 does not alter mouse brain polyunsaturated fatty acid concentrations. Lipids 45:291-299. 
Song C, Leonard BE, Horrobin DF (2004) Dietary ethyl-eicosapentaenoic acid but not soybean oil reverses central interleukin-1-induced changes in behavior, corticosterone and immune response in rats. Stress 7:43-54.

Spector AA (2006) Essential Fatty Acids. In: Biochemical, Physiological \& Molecular Aspects of Human Nutrition, 2nd Edition (Stipanuk M, ed): W.B Saunders.

Sprecher H (2000) Metabolism of highly unsaturated n-3 and n-6 fatty acids. Biochim Biophys Acta 1486:219-231.

Stafstrom CE, Vining EP, Rho JM (2008) Ketogenic Diet. In: Epilepsy: A Comprehensive Textbook, 2nd Edition (Engel J, Pedley TA, eds). Philadelphia: Lippincott, Williams \& Wilkins.

Stefanello S, Marin-Leon L, Fernandes PT, Li LM, Botega NJ (2010) Psychiatric comorbidity and suicidal behavior in epilepsy: a community-based case-control study. Epilepsia 51:1120-1125.

Svennerholm L (1968) Distribution and fatty acid composition of phosphoglycerides in normal human brain. J Lipid Res 9:570-579.

Taber L, Chiu CH, Whelan J (1998) Assessment of the arachidonic acid content in foods commonly consumed in the American diet. Lipids 33:1151-1157.

Taha AY, Ryan MA, Cunnane SC (2005) Despite transient ketosis, the classic high-fat ketogenic diet induces marked changes in fatty acid metabolism in rats. Metabolism 54:1127-1132.

Taha AY, Baghiu BM, Lui R, Nylen K, Ma DW, Burnham WM (2006) Lack of benefit of linoleic and alphalinolenic polyunsaturated fatty acids on seizure latency, duration, severity or incidence in rats.

Epilepsy Res 71:40-46.

Taha AY, Huot PS, Reza-Lopez S, Prayitno NR, Kang JX, Burnham WM, Ma DW (2008) Seizure resistance in fat-1 transgenic mice endogenously synthesizing high levels of omega-3 polyunsaturated fatty acids. J Neurochem 105:380-388.

Taha AY (2009) Anticonvulsant effects of omega-3 polyunsaturated fatty acids in rodents. Ph.D.

Taha AY, Filo E, Ma DW, McIntyre Burnham W (2009a) Dose-dependent anticonvulsant effects of linoleic and alpha-linolenic polyunsaturated fatty acids on pentylenetetrazol induced seizures in rats. Epilepsia 50:72-82.

Taha AY, Henderson ST, Burnham WM (2009b) Dietary enrichment with medium chain triglycerides (AC-1203) elevates polyunsaturated fatty acids in the parietal cortex of aged dogs: implications for treating age-related cognitive decline. Neurochem Res 34:1619-1625.

Taha AY, Jeffrey MA, Taha NM, Bala S, Burnham WM (2010) Acute administration of docosahexaenoic acid increases resistance to pentylenetetrazol-induced seizures in rats. Epilepsy Behav 17:336-343. 
Takeuchi T, Iwanaga M, Harada E (2003) Possible regulatory mechanism of DHA-induced anti-stress reaction in rats. Brain Res 964:136-143.

Tellez-Zenteno JF, Dhar R, Wiebe S (2005) Long-term seizure outcomes following epilepsy surgery: a systematic review and meta-analysis. Brain 128:1188-1198.

Tellez-Zenteno JF, Dhar R, Hernandez-Ronquillo L, Wiebe S (2007a) Long-term outcomes in epilepsy surgery: antiepileptic drugs, mortality, cognitive and psychosocial aspects. Brain 130:334-345.

Tellez-Zenteno JF, Patten SB, Jette N, Williams J, Wiebe S (2007b) Psychiatric comorbidity in epilepsy: a population-based analysis. Epilepsia 48:2336-2344.

Tso P, Balint JA (1986) Formation and transport of chylomicrons by enterocytes to the lymphatics. Am J Physiol 250:G715-726.

Venna VR, Deplanque D, Allet C, Belarbi K, Hamdane M, Bordet R (2009) PUFA induce antidepressantlike effects in parallel to structural and molecular changes in the hippocampus.

Psychoneuroendocrinology 34:199-211.

Voskuyl RA, Vreugdenhil M, Kang JX, Leaf A (1998) Anticonvulsant effect of polyunsaturated fatty acids in rats, using the cortical stimulation model. Eur J Pharmacol 341:145-152.

Vreugdenhil M, Bruehl C, Voskuyl RA, Kang JX, Leaf A, Wadman WJ (1996) Polyunsaturated fatty acids modulate sodium and calcium currents in CA1 neurons. Proc Natl Acad Sci U S A 93:12559-12563.

Weylandt KH, Kang JX, Leaf A (1996) Polyunsaturated fatty acids exert antiarrhythmic actions as free acids rather than in phospholipids. Lipids 31:977-982.

Wilder RM, Winter MD (1922) The threshold of ketogenesis. J Biol Chem 52:9.

Willis S, Samala R, Rosenberger TA, Borges K (2009) Eicosapentaenoic and docosahexaenoic acids are not anticonvulsant or neuroprotective in acute mouse seizure models. Epilepsia 50:138-142.

Wlaz P, Loscher W (1997) Anticonvulsant activity of felbamate in amygdala kindling model of temporal lobe epilepsy in rats. Epilepsia 38:1167-1172.

Wolf G (1971) Elementary Histology for Neuropsychologists. In: Methods in Psychobiology (Myers RD, ed). London: Academic Press inc.

Xiao Y, Li X (1999) Polyunsaturated fatty acids modify mouse hippocampal neuronal excitability during excitotoxic or convulsant stimulation. Brain Res 846:112-121.

Xiao YF, Kang JX, Morgan JP, Leaf A (1995) Blocking effects of polyunsaturated fatty acids on $\mathrm{Na}+$ channels of neonatal rat ventricular myocytes. Proc Natl Acad Sci U S A 92:11000-11004. 
Xiao YF, Wright SN, Wang GK, Morgan JP, Leaf A (1998) Fatty acids suppress voltage-gated Na+ currents in HEK293t cells transfected with the alpha-subunit of the human cardiac $\mathrm{Na}+$ channel. Proc Natl Acad Sci U S A 95:2680-2685.

Xu XP, Erichsen D, Borjesson SI, Dahlin M, Amark P, Elinder F (2008) Polyunsaturated fatty acids and cerebrospinal fluid from children on the ketogenic diet open a voltage-gated $\mathrm{K}$ channel: a putative mechanism of antiseizure action. Epilepsy Res 80:57-66.

Yehuda S, Carasso RL, Mostofsky DI (1994) Essential fatty acid preparation (SR-3) raises the seizure threshold in rats. Eur J Pharmacol 254:193-198.

Yehuda S, Brandys Y, Blumenfeld A, Mostofsky DI (1996) Essential fatty acid preparation reduces cholesterol and fatty acids in rat cortex. Int J Neurosci 86:249-256.

Young C, Gean PW, Chiou LC, Shen YZ (2000) Docosahexaenoic acid inhibits synaptic transmission and epileptiform activity in the rat hippocampus. Synapse 37:90-94.

Yuen AW, Sander JW, Fluegel D, Patsalos PN, Bell GS, Johnson T, Koepp MJ (2005) Omega-3 fatty acid supplementation in patients with chronic epilepsy: a randomized trial. Epilepsy Behav 7:253-258.

Zupec-Kania BA, Spellman E (2008) An overview of the ketogenic diet for pediatric epilepsy. Nutr Clin Pract 23:589-596. 